\title{
METHAMPHETAMINE SELF-ADMINISTRATION: NEUROCHEMICAL CONSEQUENCES AND BEHAVIOURAL EFFECTS
}

BY

CALEB CARATI

A thesis

submitted to the Victoria University of Wellington in fulfilment of the requirements for the degree of Doctor of Philosophy

Victoria University of Wellington

2012 


\section{Acknowledgements}

First and foremost I must thank my parents for their unwavering support, optimism, consolation, comfort and confidence throughout each and every turn of this process. To my father, Peter: thank you for always being right behind me. To my mother, Kerry: thank you for always knowing I could do it.

Without the guidance, expertise, support and iron-fisted encouragement of my manager and supervisor Professor Susan (Sioux) Schenk this would simply not have come together. You taught me so much of what it means to be a scientist both in, and out of the laboratory. I thank you most sincerely for everything you have done for me over the course of this enterprise.

The greater lab crew and close friends were all part of my postgraduate adventure and I am forever thankful for being able to share this journey with you. Special thanks to Amy Walsh, who always cheered me up and on, Sarah Braddles Bradbury, Alex Shovels Crowther, Alex Howard, Dr Joyce Colussi-Mas, Dr Matty Gerrie, Dr Dave Gittings, Dr Lincoln Hely, and, of course, the eternally stoic rock that is Richard Moore. There were many more who came along for the ride and I appreciate your assistance and distraction immensely.

Finally, I am eternally grateful to my unofficial mentor, Dr Katie Brennan. You helped me through the hardest times, carried me along the waves of triumph, answered the silly and the not so silly questions, and showed me how to do so much of what needed to be done. Not only that, but you did so with a gracious patient smile on your face and with the fantastic poise that continuously impresses me. I thank you deeply. 


\begin{abstract}
It has been suggested that methamphetamine (MA) self-administration is dependent on dopaminergic mechanisms, and that exposure to high doses of methamphetamine is toxic to central dopamine (DA) and serotonin (5-HT) neurons. Most studies, however, have utilised a short duration, high dose, experimenteradministered MA exposure regime, which is not representative of exposure that results from MA use in humans. The present studies sought to investigate the effects of self-administered MA on brain monoamine levels following a short and longer withdrawal period, and to determine the role of D1- and D2-like receptors in the maintenance of MA self-administration and in relapse to MA-seeking.

The effects of self-administered MA $(0.1 \mathrm{mg} / \mathrm{kg} /$ infusion $)$ on tissue monoamine levels were determined in rats either 24 hours or seven days following 20 daily six hour sessions. A yoked-control self-administration protocol was employed to determine the effects of response contingency.

The effect of pre-treatment with the D1-like receptor antagonist, SCH 23390 $(0.0 ; 0.01 ; 0.02 \mathrm{mg} / \mathrm{kg}$; subcutaneous [SC]), or the D2-like receptor antagonist, eticlopride $(0.0 ; 0.0125 ; 0.025 ; 0.05 \mathrm{mg} / \mathrm{kg}$; intraperitoneal [IP]) on MA selfadministration reinforced according to a fixed ratio (FR) 1, and progressive ratio (PR; $0.2 \mathrm{mg} / \mathrm{kg}$ MA) schedule was determined. The effect of these pharmacological manipulations on relapse to MA-seeking was also determined. Additionally, the role of DA in drug-seeking was examined by measuring the effect of priming injections of the direct D1 receptor agonist, SKF $81297(0.0 ; 1.0 ; 2.0 ; 4.0 \mathrm{mg} / \mathrm{kg}$; IP), the direct D2 receptor agonist, quinpirole $(0.0 ; 1.0 \mathrm{mg} / \mathrm{kg}$; IP), or the DA transporter (DAT) inhibitor, GBR 12909 (0.0; 1.0; $10.0 \mathrm{mg} / \mathrm{kg}$; IP), on MA-seeking behaviour.

Self-administered MA produced a transient decrease in tissue levels of DA and an increase in DA turnover. This effect was produced at 24 hours, but not seven days following the final self-administration session. Similar effects were produced in yoked rats that received the same, non-contingent exposure to MA. Pre-treatment with SCH 23390, but not eticlopride, produced a significant alteration in the doseresponse curve of MA self-administration reinforced on an FR1 schedule, and reduced MA produced BPs on the PR schedule. MA-seeking was produced by MA,
\end{abstract}


cocaine and GBR 12909. SCH 23390 pre-treatment significantly reduced drugprimed MA-seeking, whereas eticlopride had no significant effect. Finally, neither SKF 81297, nor quinpirole significantly increased MA-seeking.

These findings suggest that self-administered MA does not produce the extensive neurotoxicity seen following high-dose experimenter-administered treatment regimes. The finding that pre-treatment with a D1-, but not a D2-like receptor antagonist altered the maintenance of MA self-administration suggests that neuroadaptations take place as a function of MA self-administration, rendering this behaviour more reliant on D1-like receptor mechanisms. This idea is further supported by the finding that a D1-, but not a D2-like antagonist reduced drugprimed MA-seeking, and that priming injections with a D2 agonist failed to increase MA-seeking behaviour. These results are in contrast to the literature on selfadministration and reinstatement of drug-seeking following self-administration of other drugs of abuse, and suggest that dependence on different drugs may become mediated by different DA receptor mechanisms. 


\section{Table of Contents}

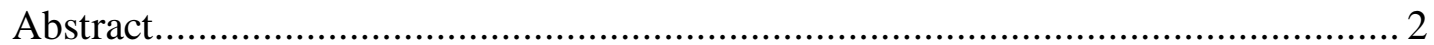

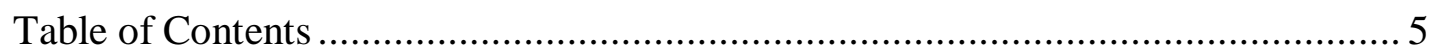

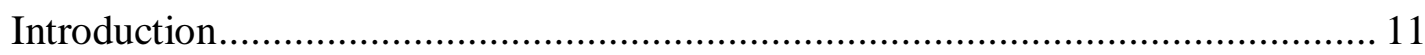

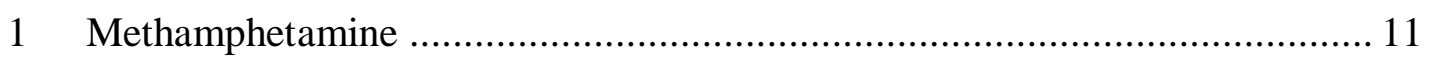

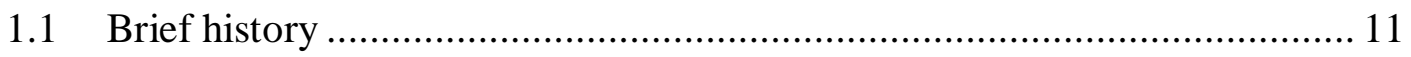

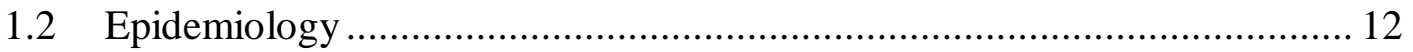

1.3 Pharmacology and pharmacokinetics of MA.......................................... 12

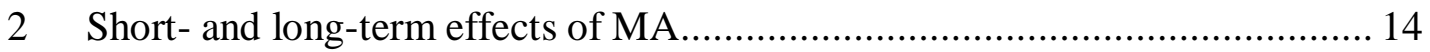

2.1 Measuring Effects of Repeated Exposure to MA …................................. 16

2.2 Effects of response contingency on effects of repeated MA exposure ......... 18

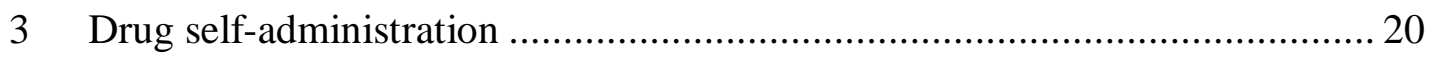

3.1 Fixed ratio schedules of reinforcement ................................................. 22

3.2 Progressive ratio schedules of reinforcement ........................................... 27

4 Neural mechanisms of drug self-administration.......................................... 34

4.1 Dopamine receptors and drug self-administration .................................. 41

5 Relapse and reinstatement of drug-seeking ........................................... 44

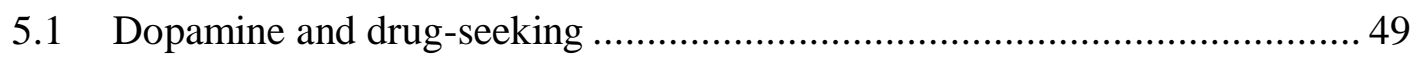

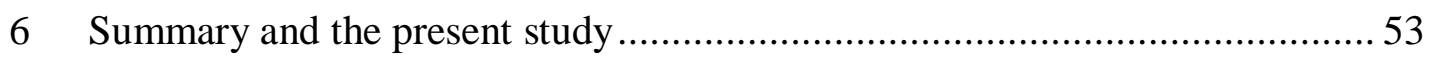

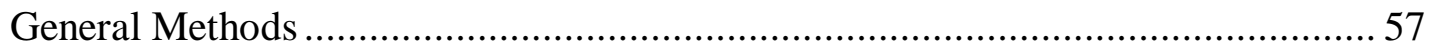

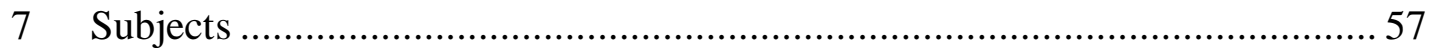

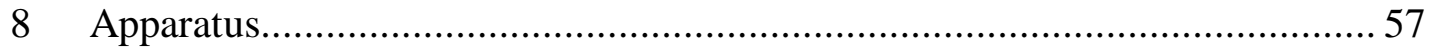

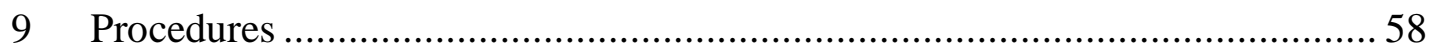

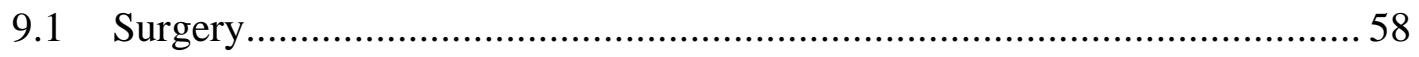

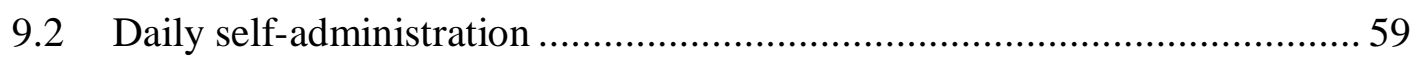

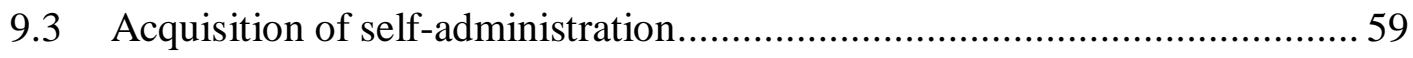

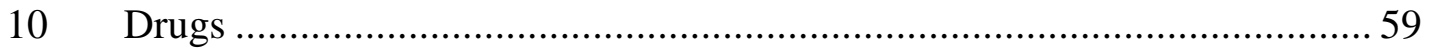

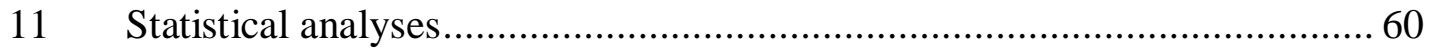

Chapter 1: Effects of MA self-administration on monoamine and metabolite tissue concentrations

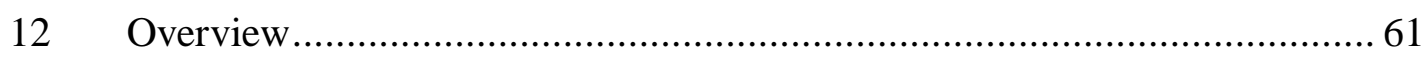


12.1 Contingency of drug exposure 63

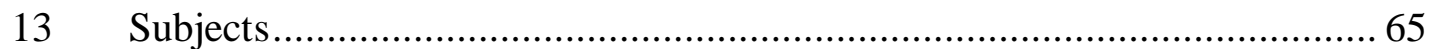

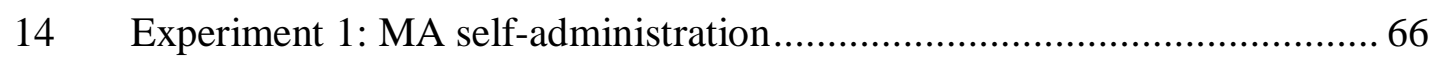

14.1 Yoked methamphetamine self-administration ........................................6 66

15 Experiment 2: Experimenter-administered MA regimen ...........................6 66

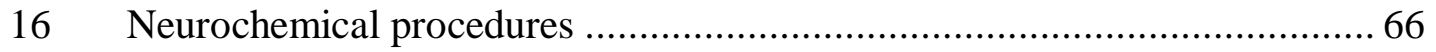

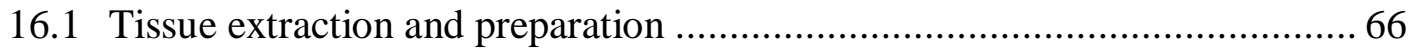

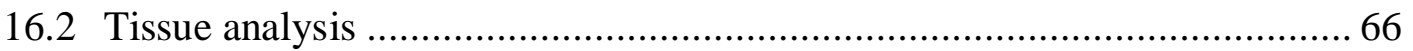

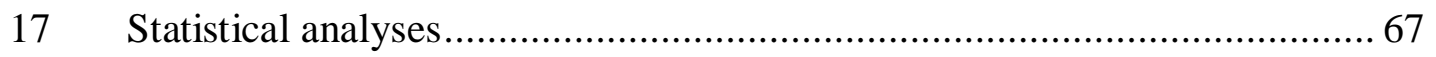

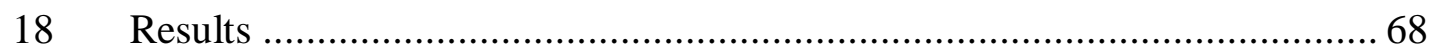

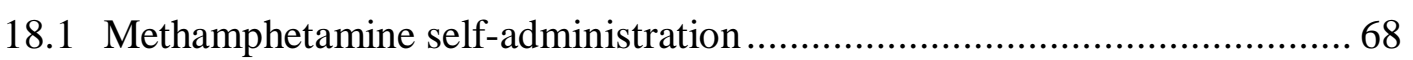

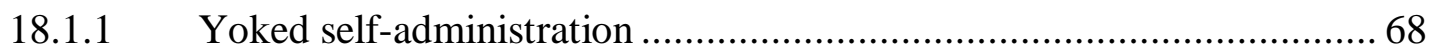

18.2 Tissue concentrations of monoamines and metabolites ...........................69

18.2.1 Self-administered/yoked MA exposure ............................................. 69

18.2.2 Experimenter-administered MA ….............................................. 70

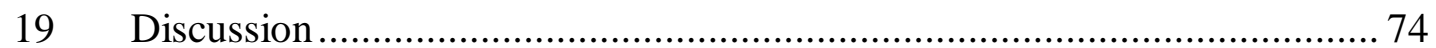

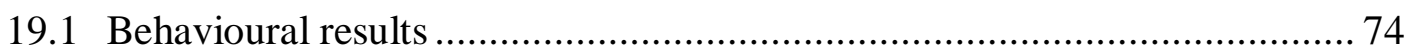

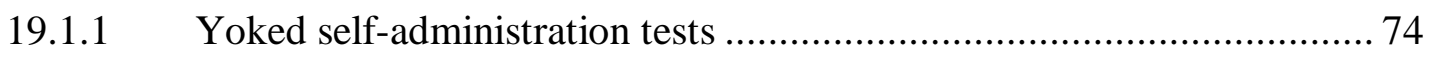

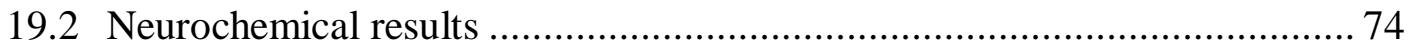

19.2.1 Experimenter-administered MA exposure …..................................... 79

19.2.2 Chronic self-administration exposure ............................................. 74

19.2.3 Yoked self-administration exposure ................................................. 76

Chapter 2: Effects of D1-like and D2-like receptor antagonists on MA self-

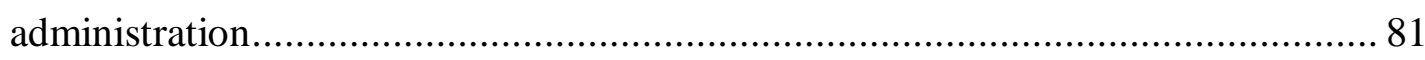

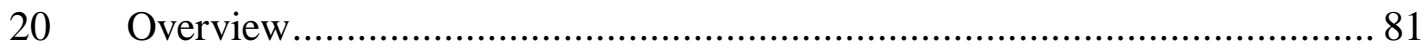

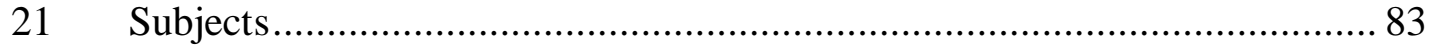

22 Experiment 3: Effects of D1- and D2-like Receptor Antagonists on Methamphetamine Self-administration on an FR1 Schedule of reinforcement........ 83

23 Experiment 4: Effects of D1- and D2-like Receptor Antagonists on Methamphetamine Self-administration on a PR Schedule of reinforcement .......... 84

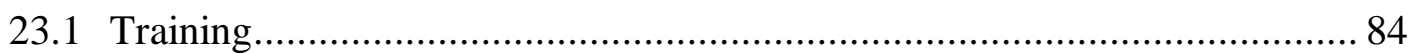

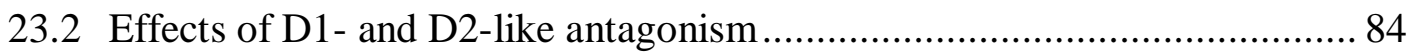

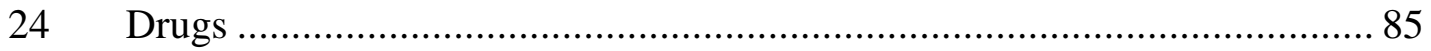

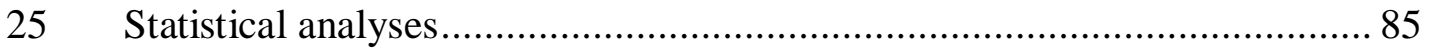


26 Results 86

26.1 Responding on the FR1 schedule of reinforcement 86

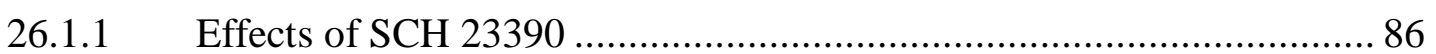

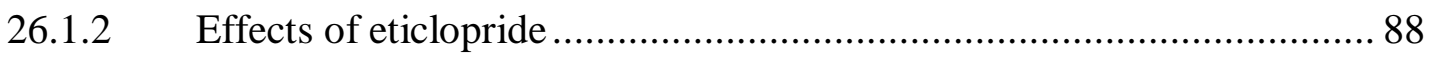

26.2 Responding on the PR schedule of reinforcement ................................... 89

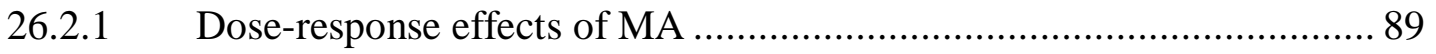

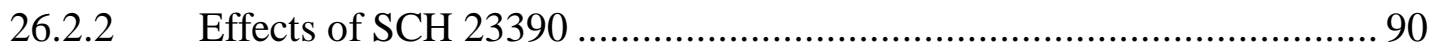

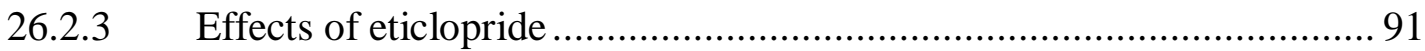

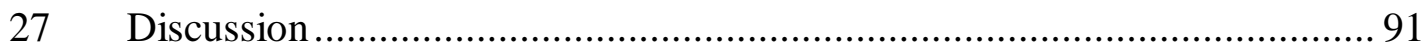

Chapter 3: Role of D1- and D2-like receptor mechanisms in drug-seeking following

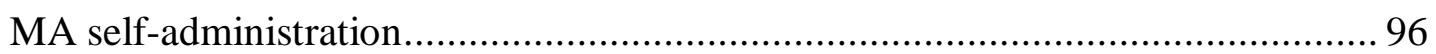

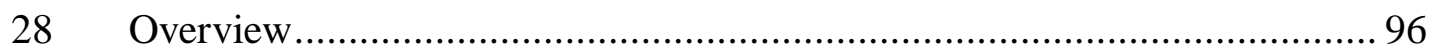

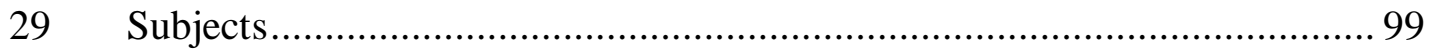

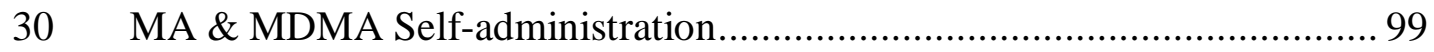

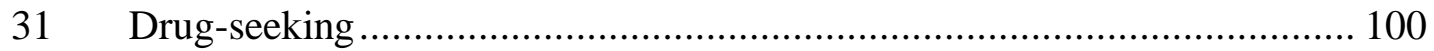

31.1 Experiment 5: MA-produced drug-seeking ........................................... 101

31.2 Experiment 6: Effects of D1- and D2-like antagonism on drug-primed drug-

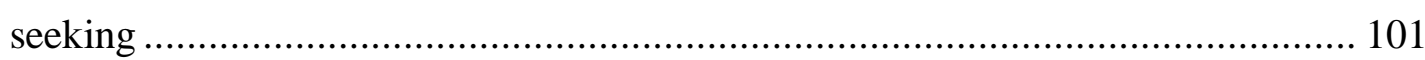

31.3 Experiment 7: DA agonist-primed MA-seeking ….............................. 102

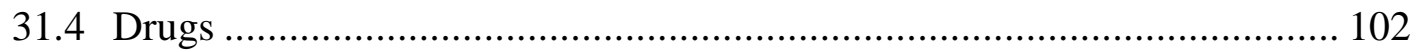

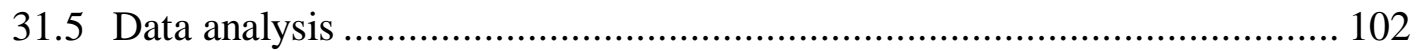

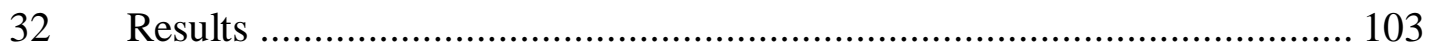

32.1 Self-administration and extinction responding .................................. 103

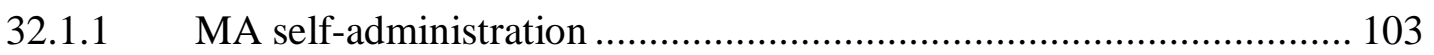

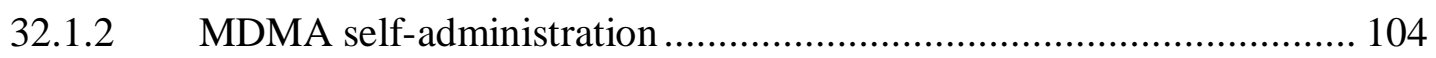

32.2 Experiment 5: MA-produced drug-seeking ......................................... 105

32.3 Experiment 6: Effects of D1- and D2-like antagonism on drug-primed drug-

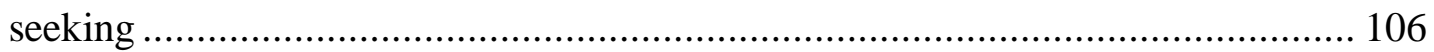

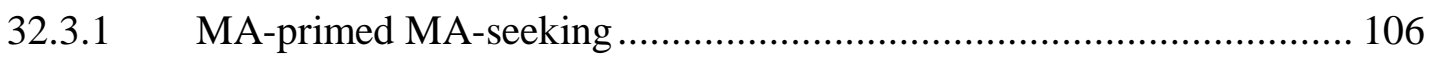

32.3.2 Cocaine-primed MA-seeking ....................................................... 108

32.3.3 MDMA-primed MDMA-seeking ..................................................... 109

32.4 Experiment 7: DA agonist-primed MA-seeking .................................. 110

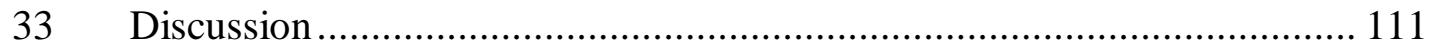

33.1 D1- and D2-like antagonist effects on MA-seeking................................. 112 
33.2 DA agonist effects on MA-seeking 114

General discussion 118

References.

\section{Table of Figures \& Tables}

Table 1. A depiction of sample timelines of within-session, between-withinsession, and between-session reinstatement procedures.

Table 2. Average $( \pm \mathrm{SEM})$ monoamine and metabolite levels $(\mathrm{ng} / \mathrm{mg}$ tissue) for the yoked vehicle (YV) groups contingent MA (CM) groups, and yoked MA (YM) groups at 24 hours and seven days following final self-administration session. Symbols show a significant difference relative to YV group: $*(p<0.05) ; \ddagger(p<$ $0.001)$. Error! Bookmark not defined.

Table 3. Average $( \pm \mathrm{SEM})$ monoamine and metabolite levels (ng/mg tissue) for the saline and methamphetamine (MA) pre-treated groups. Symbols show a significant difference relative to saline groups: $*(p<0.05) ; \dagger(p<0.01) ; \ddagger(p<$ $0.001)$

Error! Bookmark not defined.

Figure 1. Chemical structure of amphetamine and MA. 13

Figure 2. Sample graph of mean (+SEM) number of responses per session (solid line) and mean drug intake per session (broken line) as functions of unit dosage of self-administered drug.

Figure 3. Sample graph of mean (+SEM) BPs reinforced by drug selfadministration according to a PR schedule of reinforcement as a function of unit drug dose.

Figure 4. Triadic yoked MA self-administration. Contingent MA (CM; left) is delivered according to operant responding. Yoked MA (YM; centre) is delivered on the same frequency and pattern to the yoked rat dependent on responding of the rat receiving $\mathrm{CM}$. Yoked vehicle (YV; right) infusions are delivered to the second yoked rat also dependent on CM delivery. A computer controlled syringe pump delivers all CM, YM and YV infusions. Image adapted from Haracz, Mash and Sircar (1999).

Figure 5. Average number of responses $(+\mathrm{SEM})$ on inactive and active levers for $0.1 \mathrm{mg} / \mathrm{kg} / \mathrm{inf}$. MA during 6-hour sessions over a 20 day period. A) The 
contingent MA group (CM; $n=16)$. B) The yoked MA group (YM; $n=16)$. C) The yoked vehicle group ( $\mathrm{YV} ; n=16)$. *Significant difference between active and inactive levers $(p<0.05)$ 68

Figure 6. Effects of two doses of SCH 23390 [A) 0.01 ; B) $0.02 \mathrm{mg} / \mathrm{kg}$ ] on lever responding maintained by various doses of MA $(0.05,0.1,0.2 \mathrm{mg} / \mathrm{kg} / \mathrm{infusion})$. Symbols represent mean number of responses (+SEM). *Significant difference between vehicle and SCH 23390 pre-treated groups $(p<0.05)$. 86

Figure 7. Effects of three eticlopride doses [A) $0.0125 \mathrm{mg} / \mathrm{kg}$; B) 0.025 $\mathrm{mg} / \mathrm{kg}$; C) $0.05 \mathrm{mg} / \mathrm{kg}$ ] on lever responding maintained by various doses of MA $(0.05,0.1,0.2 \mathrm{mg} / \mathrm{kg} /$ infusion $)$. Symbols represent the mean number of responses $(+$ SEM). .88

Figure 8. Mean (+SEM) BPs maintained by various doses of MA $(0.05,0.1$, $0.2 \mathrm{mg} / \mathrm{kg} /$ infusion). *Significantly different from vehicle maintained BPs $(p<$ $0.05)$ 89

Figure 9. The effect of pre-treatment with various doses of SCH 23390 (0.01, $0.02,0.04 \mathrm{mg} / \mathrm{kg})$ on mean BPs (+SEM) maintained by MA $(0.2 \mathrm{mg} / \mathrm{kg} /$ infusion$)$. *Significantly different to vehicle pre-treatment $(p<0.05)$. 90

Figure 10. The effect of pre-treatment with various doses of eticlopride $(0.0125,0.025,0.05 \mathrm{mg} / \mathrm{kg})$ on mean BPs $(+\mathrm{SEM})$ maintained by MA $(0.2$ $\mathrm{mg} / \mathrm{kg} /$ infusion).

Figure 11. Average (+SEM) responding for MA self-administration on active (black bars), and inactive (grey bars) levers during two days of maintenance (Phase 1 ), and the last two days of extinction (Phase 2; FR5; $\mathrm{n}=10$ ).

Figure 12. Average (+SEM) responding for MDMA self-administration on active (black bars), and inactive (grey bars) levers during two days of maintenance (Phase 1), and the last two days of extinction (Phase 2; FR5; $\mathrm{n}=10$ ). 104

Figure 13. Average responding $(+\mathrm{SEM})$ in drug-seeking tests elicited by various doses of MA $(0 ; 1.0 ; 2.0 \mathrm{mg} / \mathrm{kg})$. $*$ Significantly different to vehicle dose $(p$ $<0.05)$. 105

Figure 14. Effects of SCH 23390 [A) 0.0; 0.01; 0.02; 0.04], and eticlopride [B) $0.0 ; 0.0125 ; 0.025 ; 0.05 \mathrm{mg} / \mathrm{kg}$ ] on drug-seeking produced by MA $(2.0 \mathrm{mg} / \mathrm{kg})$ priming. *Significantly different compared to vehicle $(p<0.05)$ 106 
Figure 15. Effects of SCH 23390 [A) 0.0; 0.01; 0.02; 0.04], and eticlopride [B) $0.0 ; 0.0125 ; 0.025 ; 0.05 \mathrm{mg} / \mathrm{kg}$ ] on MA-seeking produced by cocaine $(20.0$ $\mathrm{mg} / \mathrm{kg})$ priming. *Significantly different compared to vehicle $(\mathrm{p}<0.05)$ 108

Figure 16. Effect of eticlopride $(0.0 ; 0.0125 ; 0.025 ; 0.05 \mathrm{mg} / \mathrm{kg})$ on drugseeking produced by MDMA (10.0 mg/kg) priming. 109

Figure 17. Effect of priming injections of various DA agonists [A) quinpirole $(0.0,1.0 \mathrm{mg} / \mathrm{kg})$, B) GBR 12909 (0.0, 1.0, $10.0 \mathrm{mg} / \mathrm{kg})$, C) SKF 81297 (1.0, 2.0, 4.0 $\mathrm{mg} / \mathrm{kg}$ )] on MA-seeking behaviour. 110 


\section{Introduction}

\section{Methamphetamine}

Methamphetamine (MA) is a highly addictive drug that has devastating effects on those who become dependent. Dependence on MA, like all psychoactive drugs, is characterized by tolerance, withdrawal upon a short period of abstinence, preoccupation with MA and thoughts of acquiring it, an inability to reduce use, and continued use despite significant social, health and/or psychological impairment (APA, 2000). Tolerance to the subjective effects of MA can lead to a transition from non-injecting routes of administration to injecting MA, more frequent and longer use (Darke, Kaye, McKetin, \& Duflou, 2008; Degenhardt, et al., 2008). Injecting MA can increase the risk of blood-borne virus transmission, but also through risky sexual behaviour, as MA increases sexual arousal, and reports have shown that half of MA users are more likely to engage in high-risk sexual activities while intoxicated on MA (Darke, et al., 2008; Gonzales, Mooney, \& Rawson, 2010). Indicative of its high potential for addiction is the finding that MA abuse and dependence has been increasing across the globe - it is now considered to be one of the most consumed illicit drugs world-wide, second only to cannabis (UNODC, 2010).

\subsection{Brief history}

Originally synthesized in 1919 as a synthetic substitute for ephedrine (Buxton \& Dove, 2008), MA was initially sold over the counter in Japan to 'fight sleepiness and enhance vitality' (Anglin, Burke, Perrochet, Stamper, \& DawudNoursi, 2000). During World War II Japan, Germany, and the US issued the drug to combat personnel to increase endurance and performance (Gonzales, et al., 2010). It wasn't until the post-war period that widespread abuse began to occur, as surplus military stock flooded the market, leading to Japan's so-called "First Epidemic" (1945-1957; Anglin, et al., 2000). Use of MA slowly spread to blue-collar workers as well as students, house-wives and office workers creating what has been called Japan's "Second Epidemic" of MA abuse stretching from 1970 to the present day (Anglin, et al., 2000).

In the United States, a liquid form of MA was being used to treat heroin addiction in the 1960s, which quickly led to intravenous (IV) abuse. By the 1980s, 
MA was being illicitly manufactured by the reduction of the $\beta$-hydroxyl group on ephedrine using a mixture of iodine and red phosphorous (Cho \& Melega, 2002). This produced the crystallized, smokable and more potent form of crystal MA ("Ice", "P"; Buxton \& Dove, 2008). MA has been used therapeutically to treat a variety of disorders such as narcolepsy, attention-deficit/hyperactivity disorder (ADHD), and obesity (Berman, O'Neill, Fears, Bartzokis, \& London, 2008; Eddy,

Hallbach, Isbell, \& Seevers, 1965; G. R. Hanson \& Fleckenstein, 2009), but the growing public health threat of MA abuse and production led to the Comprehensive Methamphetamine Control Act of 1996 (Anglin, et al., 2000).

\subsection{Epidemiology}

Unlike plant based crops, such as cocaine and heroin, that depend on climate and geography for growth, a clandestine MA-lab can be established almost anywhere. The ease of production and evasion of detection has seen a spread of MA abuse in the USA from the west of the US (Hawaii, California) to rural and urban sections of the South and Midwest (Nicosia, Pacula, Kilmer, Lundberg, \& Chiesa, 2009; NSDUH, 2007). This spread of MA abuse is mirrored across the globe and the United Nations Office on Drugs and Crime: World Drug Report (UNODC, 2010) estimated between 13.7 and 59.2 million people abused amphetamine-typestimulants, including MA, in the past year, equating to $0.3-1.2 \%$ of the global population. This indicates that worldwide MA abuse is more common than cocaine and heroin abuse combined (up to 40.9 million). Rates of MA use in New Zealand exceed the global average with prevalence rates of 2.1\% among those 16-64 years of age (UNODC, 2010). MA abuse and addiction has thus become one the largest drug problems in the world.

\subsection{Pharmacology and pharmacokinetics of MA}

Compounds that contain a phenylisopropyl amine structure with different side groups can be collectively referred to as amphetamines, and MA is a structural analogue of this family. MA is synthesized in illicit laboratories by the Leukart reaction in which phenylacetone is condensed with methylamine in the presence of formic acid, producing the racemic $d / l$ compound (Cho \& Melega, 2002). A more pure and potent form of the $d$ isomer, often called "ice" or "crystal meth" due to its resemblance to ice crystals, can be produced by the reduction of the $\beta$-hydroxyl 
group on ephedrine using a mixture of red phosphorous and iodine (Cho \& Melega, 2002). Figure 1 depicts the chemical structure of amphetamine and MA.

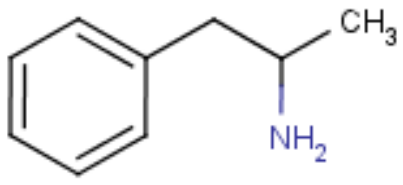

Amphetamine<smiles>CN[C@H](C)Cc1ccccc1</smiles>

Methamphetamine

Figure 1. Chemical structure of amphetamine and MA

MA is a psychostimulant that causes most of its effects on the central nervous system (CNS) through a non-exocytotic mechanism, acting as an indirect agonist for the monoamine neurotransmitters serotonin (5-HT), norepinephrine (NE) and dopamine (DA; Barr, et al., 2006; Cruickshank \& Dyer, 2009; Fleckenstein, Volz, Riddle, Gibb, \& Hanson, 2007; Julien, 2001; Kish, 2008; Sulzer, 2001; Sulzer, Sonders, Poulsen, \& Galli, 2005). Due to structural similarity with the monoamines, MA binds to membrane-bound transporters for DA (DAT), 5-HT (SERT), NE (NET) and the vesicular monoamine transporter-2 (VMAT-2). The primary mechanism by which MA increases synaptic levels of monoamines is to reverse these transporters. By reversing the action of the VMAT-2, monoamines are redistributed from synaptic vesicles into the cytosol, where they are available to be pumped into the synaptic cleft via MA-induced reverse transport of the membranebound monoamine transporters (Barr, et al., 2006; Cruickshank \& Dyer, 2009; Dwoskin \& Crooks, 2002; Elkashef, 2008; Fischer \& Cho, 1979; Fleckenstein, et al., 2007; Kish, 2008; Partilla, et al., 2006; Raiteri, Cerrito, \& Cervoni, 1979; Schmitt \& Reith, 2010; Zaczek, Culp, \& De Souza, 1991). Synaptic monoamine levels are drastically increased and are then able to stimulate monoamine receptors.

MA limits the metabolism of monoamines in the cytosol and in the synaptic cleft by inhibiting monoamine oxidase (MAO), thereby further increasing the duration and pool of monoamines available for reverse transport and the possibility of receptor activation (Barr, et al., 2006; Cruickshank \& Dyer, 2009; Elkashef, 2008; Suzuki, Hattori, Asano, Oya, \& Katsumata, 1980). The combined pharmacological 
mechanism of MA is a substantial increase in synaptic monoamine levels. Further, MA has a long elimination half-life (11-12 hours) that is greater than most other psychostimulants, producing behavioural and psychological effects lasting much longer than, for example, cocaine (1-2 hours; Julien, 2001; Newton, De La Garza II, Kalechstein, \& Nestor, 2005).

\section{Short- and long-term effects of MA}

Like other amphetamines, MA produces effects in the sympathetic nervous system similar to a "flight-or-fight" response, such as vasoconstriction, hypertension, and tachycardia (Julien, 2001). The short-term subjective effects can include pleasurable feelings of euphoria, increased alertness, motor activity and sexual drive, and decreased appetite (Caldwell, Dring, \& Williams, 1972; Cho \& Melega, 2002; Martin, Sloan, Sapira, \& Jasinski, 1971). Hyperthermia is common and high doses can induce convulsions, and can result in death (Cruickshank \& Dyer, 2009; Darke, et al., 2008).

In addition to the many detrimental effects of MA abuse to the abuser themselves; there are severe indirect damaging effects on individuals close to the abuser, and society in general. Unlike many drugs of abuse, MA appeals to both men and women equally (Gonzales, et al., 2010), and children of MA abusers run a particularly high risk of neglect and physical and emotional abuse (Anglin, et al., 2000). Costs associated with morbidity from MA overdose, criminal justice, social welfare services, environmental cleanup from MA-production, lost productivity and reduced quality of life cost the US an estimated \$23.4 billion in 2005 alone (Nicosia, et al., 2009). In NZ in 2005/06, it was estimated that $42 \%$ of social costs of illicit drug use, roughly $\$ 551$ million, were caused by illicit stimulants, such as MA (METHCON, 2008).

Following chronic MA abuse, tolerance to certain actions of MA develops, particularly the euphoric effects, leading to an increase in dose and frequency of use. The tolerance to some of MA's effects is indicative of neuronal adaptations, and may include reduced receptor binding densities, altered monoamine neurotransmission, and even neurotoxicity (Bakit, Morgan, Peat, \& Gibb, 1974; Degenhardt, et al., 2008; Martin, et al., 1971; Rogers, De Santis, \& See, 2008; Shishido, et al., 1997; Stefanski, et al., 2004; Stefanski, Ladenheim, Lee, Cadet, \& 
Goldberg, 1999). Markers of neurotoxicity, such as neuronal cell death, silver staining or reactive microgliosis, and alterations of neurotransmission have been documented, especially in dopaminergic neurons (Anglin, et al., 2000; Cho \& Melega, 2002; Derlet, Rice, Horowitz, \& Lord, 1989; J. E. Hanson, et al., 2009). For instance, magnetic resonance imaging (MRI) has revealed structural brain abnormalities in MA addicts, showing reductions in the volume of the temporal lobes (Berman, et al., 2008), and positron emission tomography (PET) scans indicate that MA abusers have significantly lower levels of DA D2-like receptor availability, compared to control subjects (Volkow, et al., 2001). A reduction in DA D2-like receptors has been linked to compulsive behaviours, such as chronic drug abuse (Everitt, et al., 2008), and the mediation of impulsive temperament (B. Lee, et al., 2009), potentially perpetuating addictive behaviours (Belin, Mar, Dalley, Robbins, \& Everitt, 2008).

MA-produced neuroadaptations can contribute to tolerance, craving and drug-seeking that are characteristic of compulsive drug dependence (APA, 2000; Cho \& Melega, 2002; Copeland \& Sorensen, 2001; Darke, et al., 2008; Hartz, Frederick-Osborne, \& Galloway, 2001). Tolerance to the euphoric effects of MA can lead to greater time and effort being spent on drug-seeking and drug-taking, along with larger doses being consumed more often, over longer periods, and selfadministration via the potentially risky IV route in an attempt to recapture the "rush" of initial use (Anglin, et al., 2000; Degenhardt, et al., 2008). This high frequency, high dose consumption pattern, with little or no sleep during a binge, can produce an extremely paranoid and irritable state. Anxiety, confusion, mood disturbances (e.g. depression, suicidal ideation), and violent, criminal behaviour can ensue (Darke, et al., 2008; Degenhardt, et al., 2008; Eddy, et al., 1965; NIDA, 2006). Psychotic features may also present, such as delusions and hallucinations (e.g. tactilehallucination of the sensation of insects crawling under the skin), which may be present months or years after cessation of drug use (Barlow \& Durand, 2002; Julien, 2001).

Studies have attempted to investigate the consequences of repeated exposure to MA in humans as the high frequency and high dose consumption have both shortand long-term effects on physiology and behaviour. Research on these effects in humans, however, are subject to ethical constraints, and are confounded by numerous issues. Patterns of illicit drug use (frequency, duration) and dosage in 
general are often obtained through self-report, and are retrospective in nature. The reliability of these reports can be questionable and is likely to be compromised given a drug's illicit status, and fragmented recall of use patterns and doses consumed (Bradburn, Rips, \& Shevill, 1987; Darke, et al., 2008). For instance, Ehrman, Robbins and Cornish (1997) demonstrated discrepancies between self-reported drug use and urine samples with two measures of self-report: simple yes/no report; and scores on the Addiction Severity Index (ASI; McLellan, et al., 1992). Positive urine samples confirmed that there was significant underreporting of cocaine use in both 48 hour self-reports, and on 30 day retrospective reports provided by the ASI (Ehrman, et al., 1997). Drug use and assessments of subsequent/previous use are often separated by substantial delay, potentially decreasing recall accuracy, and low statistical power from between-subject experimental designs may increase the likelihood of type II errors (Hartz, et al., 2001). Several routes of administration (e.g. snorting; smoking; IV injection) are popular among MA users and may alter the neurobiological consequences of exposure (Degenhardt, et al., 2008; Elkashef, 2008). Furthermore, because MA is illicitly manufactured, the dose and purity are variable (Anglin, et al., 2000; Gonzales, et al., 2010; Nicosia, et al., 2009). Polydrug use is often reported among MA users (Booth, Leukefeld, Falck, Wang, \& Carlson, 2006; Degenhardt, et al., 2008), making it difficult to ascertain whether physiological and/or psychological impairments are due to the consequences of MA exposure alone, or the combination of drugs consumed. Additionally, ethical constraints limit the ability to administer MA to naïve participants.

Animal models, however, can provide the advantage of flexibility of method, precision in measurement, reproducibility and opportunity for experimental manipulation and control of extraneous variables that permits conclusions to be made that would not be possible using human studies. These models can then be appropriately adapted to investigate the consequences of repeated, chronic MA exposure, and the neurobiological mechanisms underlying its abuse.

\subsection{Measuring Effects of Repeated Exposure to MA}

Many studies have investigated the effects of repeated MA on the brain of laboratory animals. Of great concern is that a single, or repeated, high dose exposure to MA causes neurotoxic effects, and neuronal apoptosis, particularly in dopaminergic and serotonergic neurons (Cadet, et al., 2009; Cadet, Ladenheim, \& 
Hirata, 1998; Fleckenstein, Gibb, \& Hanson, 2000; Fuller \& Hemrick-Leuke, 1980; J. E. Hanson, et al., 2009; Hotchkiss \& Gibb, 1980; Itzhak, 2004; Lorez, 1981; Melega, et al., 2008; Segal, Kuczenski, O'Neill, Melega, \& Cho, 2005). These neuroadaptations may account for the various adverse neurological deficits (Cadet, Jayanthi, \& Deng, 2003; Friedman, Castañeda, \& Hodge, 1998) and cognitive and behavioural problems seen in MA abusers (Barr, et al., 2006). High doses of MA produced persistent DA (Melega, et al., 2008; Segal, et al., 2005; Wagner, et al., 1980), and 5-HT reductions (Cadet, et al., 2009; Ricaurte, Schuster, \& Seiden, 1980) in several brain regions (Friedman, et al., 1998; Hotchkiss, Morgan, \& Gibb, 1979; Ricaurte, et al., 1980). These reductions were evident long after the final exposure (237 days), suggesting extensive neurotoxicity (Friedman, et al., 1998). The effects of MA on DA and 5-HT neurons appear to be region specific; MA-induced DA reductions are most pronounced in the dorsal striatum (Str; Chu, et al., 2008; Stephans \& Yamamoto, 1996; Wagner, et al., 1980), whereas the prefrontal cortex (PFC) and hippocampus (Hpc) appear most susceptible to 5-HT reductions (Friedman, et al., 1998; Ricaurte, et al., 1980; Stephans \& Yamamoto, 1996).

In many studies, the consequences of MA exposure have been assessed following experimenter administration. A typical dosing procedure that has produced neurotoxicity comprises four injections of $10 \mathrm{mg} / \mathrm{kg}$ MA every two hours for a total of $40 \mathrm{mg} / \mathrm{kg}$, administered to drug-naïve animals (e.g. Cadet, et al., 1998; T. L. Wallace, Gudelsky, \& Vorhees, 2001). This pattern of exposure has been justified as representative of "a naïve user overdose" (p. 189, Davidson, Lee, \& Ellinwood, 2005). The results from studies employing experimenter-administrated, repeated high-dose injection MA exposure have been crucial in exploring the neurobiological substrates that are affected by MA abuse and dependence, but may not be representative of the effects produced by the chronic MA self-administration of dependent individuals. Specifically, MA use is typically initiated at lower doses with long intervals between administrations, before progressing to higher doses with shorter intervals between administrations, and eventually multiple daily high dose administrations (Cho \& Melega, 2002; Degenhardt, et al., 2008; Fischman \& Schuster, 1974; Robinson \& Camp, 1987; Segal, Kuczenski, O'Neil, Melega, \& Cho, 2003). A number of studies have, however, attempted to administer MA in a manner that is more comparable to human abuse patterns. These include escalating dose (ED) protocols (e.g. Cadet, et al., 2009; C. J. Schmidt, Sonsalla, Hanson, Peat, \& 
Gibb, 1985; Stephans \& Yamamoto, 1996), pharmacokinetic modelling (e.g. Cho, Melega, Kuczenski, \& Segal, 2001; Herring, Schaefer, Gudelsky, Vorhees, \& Williams, 2008), passive chronic exposure using osmotic minipumps (e.g. Davidson, et al., 2005), and IV self-administration (e.g. Deneau, Yanagita, \& Seevers, 1969; Krasnova, et al., 2010; Schwendt, et al., 2009; Shepard, Chuang, Shaham, \& Morales, 2006; Weeks, 1962).

\subsection{Effects of response contingency on effects of repeated MA exposure}

ED and pharmacokinetic modelling studies may provide greater approximations of human MA abuse patterns attempting to produce similar effects. Because MA users progressively increase their drug intake, ED procedures may more accurately simulate this gradual increase in drug dose by treating naïve animals with small doses that are progressively increased (O'Neil, et al., 2006; C. J. Schmidt, et al., 1985; Segal, et al., 2003). Pharmacokinetic modelling, on the other hand, attempts to more accurately model the accumulation of drug in blood plasma by adjusting dosing intervals in laboratory animals to mimic steady-state plasma concentrations in human MA users. This is due to the significant differences in the rate of drug metabolism between humans and laboratory animals - MA elimination half-life in the rat is ca 70 minutes, and for humans ca 12 hours (Cho, et al., 2001). Greater approximations of human doses and exposure patterns can be attained using ED regimens and pharmacokinetic modelling, but they still employ a non-contingent exposure methodology whereby MA is administered by the experimenter. This may have profound effects on the consequences of that exposure, as several studies have demonstrated different effects of self- and experimenter-administered drugs on neuroadaptations (Dworkin, Co, \& Smith, 1995; Hemby, Co, Koves, Smith, \& Dworkin, 1997; Jacobs, Smit, de Vries, \& Schoffelmeer, 2003; Stefanski, et al., 2004; Stefanski, et al., 1999). For instance, Sigma1 receptor (Sig-1R) proteins were up-regulated in the midbrain, and altered levels of Sig-1R mRNA in the frontal cortex (FC) and Hpc were found only in rats self-administering MA (Stefanski, et al., 2004). There were significantly higher extracellular DA levels in the nucleus accumbens (NAc) in rats self-administering cocaine, compared to littermates receiving identical passive infusions of cocaine (Hemby, et al., 1997), and evidence suggests that non-contingent exposure to cocaine produced aversive properties in rats and reduced the incentive motivation for further drug reinforcement (Twining, 
Bolan, \& Grigson, 2009). There were also significant reductions in DA receptor binding densities in the ventral tegmental area (VTA), substantia nigra (SN) and NAc only in rats exposed to self-administered MA (Stefanski, et al., 1999). These findings suggests that the neuroadaptive changes taking place in the brain as a consequence of drug exposure depend on the exposure protocol employed (active/contingent vs. passive/non-contingent), and that studies employing experimenter-administered MA protocols may fail to obtain relevant consequences of self-administered drug exposure.

Drug self-administration procedures, in addition to a high degree of control of extraneous variables, permits investigation of the mechanisms associated with voluntary initiation of drug-taking behaviour; the maintenance of drug-taking; drugcraving and relapse to drug-seeking behaviours; and the consequences of drug selfadministration. The fundamental principle of drug self-administration adheres to the behaviourist view that behaviour is controlled by the consequences it produces (Deneau, et al., 1969; Schuster \& Thompson, 1969). Thus, drugs are selfadministered because they produce positive reinforcing effects. Animal models often focus on the ability of drugs of abuse to directly control behaviour, a critical diagnostic feature of drug dependence (APA, 2000), and have demonstrated that self-administered drug exposure is sufficient to motivate further drug-taking behaviour.

The validity of drug self-administration in laboratory animals stems from the observation that laboratory animals self-administered drugs that are abused by humans, whereas drugs that do not maintain self-administration are typically not abused by humans (Deneau, et al., 1969; Weeks, 1962; Wilson, Hitomi, \& Schuster, 1971; Wilson \& Schuster, 1972). This finding also suggests that drugs may act as universal reinforcers. That is, many of the complex chains of behaviour associated with drug abuse in humans are not necessary for the reinforcing effects to be experienced; rather, drug reinforcement appears to involve biological processes that are common to mammalian species (Lynch, Nicholson, Dance, Morgan, \& Foley, 2010). Obviously, the how and why of drug self-administration varies between a human within their social environment(s); and an animal experimental subject in a controlled laboratory setting. The neural chemistry involved in the behavioural patterns of drug-seeking, drug-taking, and its consequences, however, are similar in both situations (Sanchis-Segura \& Spanagel, 2006). Accordingly, animal models of 
drug self-administration may be able to elucidate the common neural mechanisms and therefore contribute to identifying useful strategies of intervention in human drug abuse and dependence.

\section{Drug self-administration}

"All models are wrong, but some are useful."

George Box, "Robustness in the strategy of scientific model building," in Robustness in Statistics, R. L. Launer and G. N. Wilkinson, editors. 1979, Academic Press, New York.

The study of drug addiction using drug self-administration stems from operant theory, stipulating that drugs can function as reinforcers in and of themselves and will therefore increase the likelihood of the behaviour that produces their delivery. Operant responding for drug reinforcers can become controlled in the same manner that food and water reinforcers can control the behaviour of a food or water deprived animal (Deneau, et al., 1969; Schuster \& Thompson, 1969; Wilson, et al., 1971). Drug reinforcement, however, through the neuroadaptations of repeated drug exposure on the brain, can eventually become far more potent than natural rewards, gaining the astonishing power over behaviour that is part of the dependent state (Gardner, 2000; Spealman \& Goldberg, 1978). Further, because drug exposure is behaviourally contingent, drug self-administration represents arguably the most appropriate animal model for studying the positive reinforcing and addictive properties of drugs of abuse (Stephens, Duka, Crombag, Cunningham, \& Heilig, 2010). These methods of studying drug dependence can be widely used in basic and preclinical drug abuse research as they possesses substantial construct and face validity as models of human drug consumption and provide the most direct point-topoint correspondence with human drug abuse in the natural environment (Gardner, 2008; Panlilio \& Goldberg, 2007; Sanchis-Segura \& Spanagel, 2006).

A number of routes of administration are able to maintain successful drug self-administration in the laboratory. These include IV, intramuscular, intraperitoneal (IP), and intracranial (IC) injections, in addition to oral ingestion and inhalation. The most commonly used are IV and oral. For oral self-administration studies, drinking spigots are inserted into experimental chambers with automated apparatus to measure licking behaviour, whereas for IV self-administration, chronic 
indwelling IV catheters are surgically implanted in experimental animals and attached to automated syringe pump systems (Collins, Weeks, Cooper, Good, \& Russell, 1984; Gardner, 2000; Weeks, 1962). Due to the rapid onset of effects, the IV route of administration further strengthens the association between the operant behaviour and subjective drug effects (Carlson, 2004). A variety of species selfadministered drugs of abuse (e.g. rodents, non-human primates, dogs) and these methods have demonstrated reliable self-administration of virtually all drugs of abuse including cocaine, amphetamines (including MA and MDMA), caffeine, opiates, ethanol, sedative-hypnotics (e.g. valium, xanax), dissociative anaesthetics (e.g. ketamine), and cannabinoids (Deneau, et al., 1969; Di Chiara \& Imperato, 1988; Gardner, 2000; Roberts, 1993) ${ }^{1}$.

A limitation of the drug self-administration paradigms is that they are relatively expensive in terms of resources and time. Using IV delivery, for example, demands correct and safe surgical implantation of IV catheters requiring substantial training and specialized equipment. Catheter patency must then be constantly maintained, and checked throughout a study to ensure efficient and reliable drug delivery. In the event of catheter patency failure another catheter may be inserted in the second jugular vein. This delays data collection, however, and may interfere in training schedules or experiment time frames. Further, drug self-administration studies using rodents are limited by the simple fact that the patency of IV catheters can be maintained, at best, for a few months. This precludes studies over longer periods, and the use of long, complex training schedules (Sanchis-Segura \& Spanagel, 2006).

Using animal drug self-administration as a model of human drug consumption, paradigms have been developed to ask questions about drug abuse and dependence that were not previously possible without clinical study confounds. For instance, the possible abuse liability of a compound can be evaluated by investigating whether a drug naïve animal voluntarily initiates and maintains selfadministration of that compound (Deneau, et al., 1969). Patterns of drug-taking (e.g. Downs \& Woods, 1974; Wilson, et al., 1971), response contingency effects on drug-

\footnotetext{
${ }^{1}$ For the purposes of exploring the neurobiological mechanisms involved in MA self-administration in this thesis, comparisons of self-administration behaviour will focus predominantly on other psychostimulants, particularly cocaine, as this is where the majority of research has been conducted.
} 
taking (e.g. Goldberg, 1973; Roberts, 1993), the development of addiction-like behaviours (e.g. Roberts, Morgan, \& Liu, 2007), relapse to drug-seeking and -taking (e.g. de Wit \& Stewart, 1981; Stretch \& Gerber, 1973), and the neural mechanisms involved in the reinforcing properties of drugs of abuse (e.g. Roberts, Andrews, \& Vickers, 1996) are but a number of questions that can be asked using animal models of drug self-administration.

As a method for investigating the neurobiological mechanisms of drug abuse and dependence, animal drug self-administration paradigms must be adapted and appropriately tuned in order to model the particular aspect of addiction-like behaviour that is under consideration - no single paradigm will be effective in evaluating all questions relevant to the study of MA abuse and dependence. Only by investigating MA self-administration under a number of paradigms designed to model different aspects of addiction-like behaviours can we gain a proper understanding of the neurobiological mechanisms underlying MA dependence. There are many paradigms of drug self-administration that can model several aspects of human drug dependence, but introduction and discussion will be limited to those relevant to the interpretation of the results contained herein.

\subsection{Fixed ratio schedules of reinforcement}

The great majority of drug self-administration studies have used a fixed ratio (FR) schedule of reinforcement. Under this schedule, the reinforcer (drug) is delivered every time a pre-selected number of operant responses (e.g. depression of a lever) are completed. Generally, an operant chamber may have two levers: depression of one lever results in drug delivery (active lever); whereas depression of the other may be noted, but has no pre-programmed response (inactive lever). Recording responses on the inactive lever can provide a measure of generalization of responding, and undirected behaviour. Significantly greater responding on the active lever, compared to the inactive lever, can then indicate that the self-administered compound acts as a reinforcer of the operant behaviour (lever press).

The most extensively used FR is FR1, also termed continuous reinforcement, where each response is reinforced by delivery of the drug. This schedule provides a simple association of the direct relationship between the operant behaviour and the subsequent effects of drug delivery, making this the easiest and fastest self-administration schedule to train. A drug self-administration paradigm 
using a simple FR schedule of reinforcement can provide a quick and efficient indication of whether a compound will support self-administration behaviour, providing a measure of whether that compound possesses reinforcing properties and any potential for abuse.

Weeks (1962) developed a chronically implanted IV catheter that, for the first time, provided a means of conducting long-term self-administration studies. First, experimenter-administered morphine sulphate injections were administered to rats in hourly doses, increasing in a $2.5 \%$ geometric progression from 2 to $40 \mathrm{mg} / \mathrm{kg}$ (122 doses in total), and the last dose being repeated for 1 to 2 days. This treatment regimen was suggested to produce physiological dependence. Next, a lever was inserted into the rat's cage, which, when pressed, produced a $10 \mathrm{mg} / \mathrm{kg}$ infusion of morphine sulphate. Following chance lever pressing producing the drug effects, lever pressing became regular, and response rates increased in a compensatory manner when the unit dose per infusion was reduced to $3.2 \mathrm{mg} / \mathrm{kg} /$ infusion (Weeks, 1962). This indicated that morphine sulphate infusions were reinforcing, and that rats would perform an operant behaviour in order to obtain further infusions. Further, this study highlights the ease of training and acquisition of the operant behaviour using an FR1 schedule of reinforcement.

Deneau and colleagues (1969) extended these findings by developing chronic indwelling catheters which permitted rhesus monkeys to voluntarily initiate IV selfadministration. The ability of drugs to support voluntary initiation of drug selfadministration is important in determining abuse liability, as it is possible that morphine self-administration following suggested physiological dependence (Weeks, 1962) was maintained by the negatively reinforcing amelioration of opiate withdrawal. CNS psychostimulants with abuse liability (e.g. MA), however, do not typically produce physiological dependence (Julien, 2001). A number of drugs (morphine, codeine, cocaine, $d$-amphetamine, pentobarbital, ethanol), however, induced and maintained voluntary self-administration behaviour (Deneau, et al., 1969), indicating a potential liability for abuse, irrespective of their ability to produce physiological dependence. Importantly, a number of compounds that are not abused by humans, such as nalorphine, chlorpromazine, and physiological saline, did not support self-administration behaviour, suggesting they were not intrinsically reinforcing, and did not possess similar potential for abuse (Deneau, et al., 1969). The use of voluntary initiation and maintenance of drug self-administration in 
animals is now well accepted as a test of predicting abuse liability in humans (Collins, et al., 1984).

Voluntary initiation and maintenance of MA self-administration has subsequently been widely reported, in a number of animals, including rodents and non-human primates (Brady, McCallum, Glick, \& O'Donnell, 2008; Clemens, Cornish, Hunt, \& McGregor, 2006; Deneau, et al., 1969; Harrod, Dwoskin, Crooks, Klebaur, \& Bardo, 2001; Johanson, Balster, \& Bonese, 1976; Jun \& Schindler, 2000; Kitamura, Wee, Specio, Koob, \& Pulvirenti, 2006; Ranaldi \& Poeggel, 2002; Yokel $\&$ Pickens, 1973). This supports the idea that it is positively reinforcing, and possesses potential for abuse and dependence. For instance, an early study demonstrated that experimentally naïve rats voluntarily initiated and maintained self-administration of MA under an FR1 schedule of reinforcement (Yokel \& Pickens, 1973). When self-administration was limited to $6 \mathrm{hr} / \mathrm{day}$, an increase in dose produced a decrease in rate of responding, and body weight and health was maintained in the rats. MA's immense potential for producing addictive-like behaviours was further demonstrated when animals given unlimited ( $24 \mathrm{~h} /$ day) selfadministration access showed substantial weight loss, body irritation and typically died within two weeks (Johanson, et al., 1976; Yokel \& Pickens, 1973).

The finding that an increase in the unit dose of self-administered MA resulted in a decrease in rate of responding (Yokel \& Pickens, 1973) is typical of drug self-administration behaviour patterns reinforced according to an FR schedule. Drug self-administration is governed by the dose of self-administered drug, and the rate of operant responding is an inverse function of the unit dose per drug infusion. From the threshold unit dose (mg/kg/infusion) capable of supporting selfadministration behaviour at the peak of the dose-response curve, response rates decreased in accordance with increases of the unit dose, yielding a dose-response function resembling an inverted-U shape (Deneau, et al., 1969; Downs \& Woods, 1974; Goldberg, 1973; Markou, et al., 1993; Schuster \& Thompson, 1969; Skjoldager, Winger, \& Woods, 1991; Spealman \& Goldberg, 1978; Wilson, et al., 1971; Wilson \& Schuster, 1972). For instance, the relationship between unit dose of drug infusion and rate of responding reinforced by both $d$ - and $l$ - isomers of MA and amphetamine resembled an inverted-U shape (Yokel \& Pickens, 1973). The mean duration between operant responses was directly related to the unit dose per injection for each dose that maintained responding. This means there was an inverse 
relationship between the unit dosage and rate of responding - as the selfadministered dose increased, response rates decreased (Yokel \& Pickens, 1973). More recently, self-administration behaviour was maintained by three doses of IV MA $(0.05,0.1,0.25 \mathrm{mg} / \mathrm{kg} /$ infusion), and maximum response rate was maintained by the lowest dose, followed by slower rates of responding as the unit dose increased (Jun \& Schindler, 2000). The generated dose-response function comprised the descending limb of MA self-administration, and further dose increases would produce slower response rates. This relationship has been suggested to reflect an attempt at compensation by decreased responding following greater drug effects at higher unit doses (Yokel \& Pickens, 1973), and changes in response rates may reflect an attempt to maintain a criterion amount of blood plasma drug level stimulation (Arnold \& Roberts, 1997; Richardson \& Roberts, 1996).

Given that increasing the unit dose quickly resulted in lower response rates and fewer infusions, early drug self-administration studies found that total overall session drug intake remained relatively stable, independent of dose variations (Deneau, et al., 1969; Downs \& Woods, 1974; Goldberg, 1973; Wilson, et al., 1971; Yokel \& Pickens, 1973). Indeed, increasing the unit dose of MA, amphetamine, cocaine, pipradrol, methylphenidate, and phenmetrazine produced slower response rates, and consequently, little change in overall session drug intake (Wilson, et al., 1971; Yokel \& Pickens, 1973). At doses that maintained self-administration behaviour, no significant differences were found between drug intake per session across doses for both amphetamine and MA (Yokel \& Pickens, 1973). The most parsimonious explanation for dose-dependent responding is that lower rates of responding at higher doses reflect a compensatory mechanism to maintain optimum blood levels of drug - the magnitude of drug effect is maintained at a relatively constant level by increasing drug intake in the face of reduced drug impact and vice versa. In other words, drug self-administration on FR schedules is rapidly titrated in response to changes in dose, in a similar manner to peripheral mechanisms regulating self-administration of natural, non-drug reinforcers such as food and water (Gardner, 2000, 2008; Wilson, et al., 1971). Thus, rate of responding is a direct function of the unit dose being self-administered, whereas total drug intake per session remains relatively stable across doses. It should be emphasized, however, that one of the fundamental aspects of drug dependence is that drug intake can become uncontrollable and can lead to death by overdose (APA, 2000), even in 
animals (Johanson, et al., 1976; Yokel \& Pickens, 1973). Therein lies the distinction between 'recreational' use, where intake is controlled, and abuse, where control has been lost. Figure 2 depicts the descending limb of a sample dose-response curve for active lever responding and overall total session drug intake as functions of selfadministered unit drug dose.

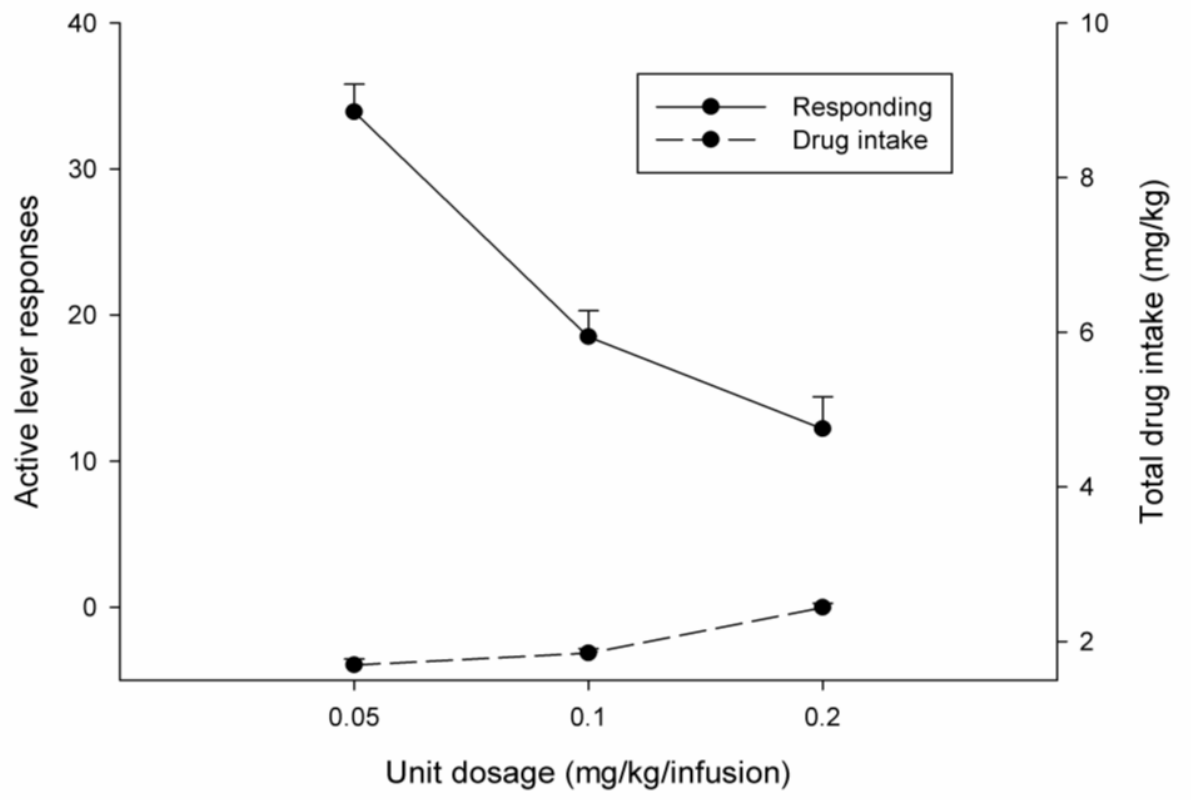

Figure 2. Sample graph of mean (+SEM) number of responses per session (solid line) and mean drug intake per session (broken line) as functions of unit dosage of self-administered drug.

The characteristic inverted U-shape of the dose-response curve for drug selfadministration, producing stable amounts of drug-intake across doses, can provide important insight into patterns of drug-taking behaviour. By plotting drug selfadministration across doses, changes in the dose-response curve may be suggestive of changes in the drug's pharmacological effects. Figure 2 shows that, on the descending limb of the dose-response curve, higher unit doses of self-administered drug produce lower rates of responding, whereas lower unit doses produce higher response rates, in a compensatory manner. The opposite is true for doses on the ascending limb - namely, increases in dose would produce increases in response rates. This suggests that on the descending limb lower response rates may reflect increased positive reinforcing effects and vice versa. This idea was supported by the finding that systemic partial DA blockade with low doses of pimozide or (+)butaclamol increased the rate of responding maintained by amphetamine, whereas pre-treatment with higher doses decreased response rates (Yokel \& Wise, 1976). 
Amphetamine's effects are mediated by non-exocytotic indirect agonism of the monoamines (DA, NE, 5-HT; Carlson, 2004; Julien, 2001) by blocking and reversing the action of membrane-bound monoamine transporters, in a similar mechanism to MA. Thus, pre-treatment with low doses of pimozide and/or (+)butaclamol, partially blocking amphetamine's effects at DA receptors, produced increased rates of responding suggesting a rightward shift in the dose-response curve (Yokel \& Wise, 1976). This increase in response rate potentially compensated for a reduction in amphetamine's pharmacological efficacy. This idea was further supported by a cessation of responding at full blockade of DA receptors by higher doses of pimozide and (+)-butaclamol (Yokel \& Wise, 1976), suggesting a complete blockade of the positive reinforcing effects of amphetamine.

In terms of training drug self-administration in animals, responding on simple FR schedules of reinforcement represents the easiest method. As such, the voluntary initiation and acquisition of this behaviour can provide important insight into the potential abuse liability of a compound. Further, the maintenance of drug self-administration, across several unit doses of drug, can be used to generate a doseresponse curve to investigate patterns of drug-taking behaviour, and changes in a drug's reinforcing effects. With different doses producing different rates of responding, response rate being inversely related to unit dose, and overall drugintake remaining relatively stable across dose variations, dose-dependent responding likely reflects a compensatory mechanism to maintain a preferred level of drug stimulation. This is valuable for interpreting changes in rate of responding following experimental manipulations. Responding reinforced according to an FR schedule, however, does not tell us much in the way of the neurobiological mechanisms underlying the incentive motivation, or "drive", for further drug reinforcement that is so characteristic of drug abuse and dependence. Further, one of the greatest obstacles to overcome in drug rehabilitation treatment is relapse to drug abuse, even following long periods of abstinence. Drug self-administration on other schedules of reinforcement has been used to address these aspects of drug-taking and drugseeking.

\subsection{Progressive ratio schedules of reinforcement}

Progressive ratio (PR) schedules of reinforcement were originally developed to measure the reinforcing efficacy of condensed milk solutions in rats (Hodos, 
1961), and have since been exploited for drug self-administration studies (Bedford, Bailey, \& Wilson, 1978; Gardner, 2000, 2008; Griffiths, Bradford, \& Brady, 1979; Panlilio \& Goldberg, 2007; Richardson \& Roberts, 1996; Roberts, 1993; SanchisSegura \& Spanagel, 2006; Stafford, LeSage, \& Glowa, 1998). The PR schedule of reinforcement as a measure of reinforcing efficacy has been used with all species of experimental animals including non-human primates, dogs, mice and rats. Under this schedule of reinforcement, the response requirements for each drug infusion increase according to a preset series, to the point where the operant behaviour is no longer performed, or does not meet the required response criterion within a stipulated timeframe (e.g. one hour). In this manner, the maximum response requirement that will support self-administration behaviour, termed the break-point (BP), can be established. The BP is intended to reflect the incentive motivation for further drug reinforcement, and can be interpreted as a measure of reinforcing efficacy of the self-administered drug. The BP measure has been demonstrated as stable over days and across studies. Different laboratories using a PR schedule of reinforcement have reported concordant findings - higher unit doses support higher BPs (Arnold \& Roberts, 1997; Markou, et al., 1993; Panlilio \& Goldberg, 2007; Richardson \& Roberts, 1996; Roberts, et al., 2007; Sanchis-Segura \& Spanagel, 2006). The completion of high ratios (e.g. > 1000) for a single drug infusion on a PR schedule of reinforcement has been compared to the extremely high motivation for further drug-taking seen in human drug users; corresponding to the DSM-IV-TR criteria for substance dependence of spending a great deal of time in activities necessary to obtain and/or use the substance (APA, 2000; Deroche-Gamonet, Belin, \& Piazza, 2004).

In contrast to FR schedules of reinforcement, the dependent variable on a PR schedule of reinforcement is not related to the rate of responding, but rather is the absolute highest ratio completed - the time it takes to complete each ratio does not matter (within the temporal limits of the paradigm), just whether or not the ratio is completed. Because BPs are independent of the rate of responding, the BP can provide an effective measure of the reinforcing efficacy of a self-administered drug, and incentive motivation for further drug reinforcement, complementing interpretations of any changes in response rates observed under an FR schedule of reinforcement. 
Hodos (1961) demonstrated that higher concentrations of condensed milk generated higher BPs, suggesting that the higher concentrations were more reinforcing. This effect has also been demonstrated for drugs of abuse - higher unit doses will support more persistent self-administration behaviour and sustain higher BPs, suggesting greater reinforcing efficacy. Dose-dependent responding reinforced according to a PR schedule generates a positive linear dose-response function. Indeed, BPs produced during MA self-administration in rats (Brady, et al., 2008; Clemens, et al., 2006; Ranaldi \& Poeggel, 2002; Roth \& Carroll, 2004; Wee, Wang, Woolverton, Pulvirenti, \& Koob, 2007), and rhesus monkeys (Wang \& Woolverton, 2007) were dose-dependent, with higher unit doses supporting higher BPs. Higher BPs indicate that the higher doses of MA are more reinforcing, and reflect a higher incentive motivation for further MA reinforcement (Arnold \& Roberts, 1997; Markou, et al., 1993; Richardson \& Roberts, 1996). Figure 3 depicts a sample doseresponse function of drug self-administration on a PR schedule of reinforcement.

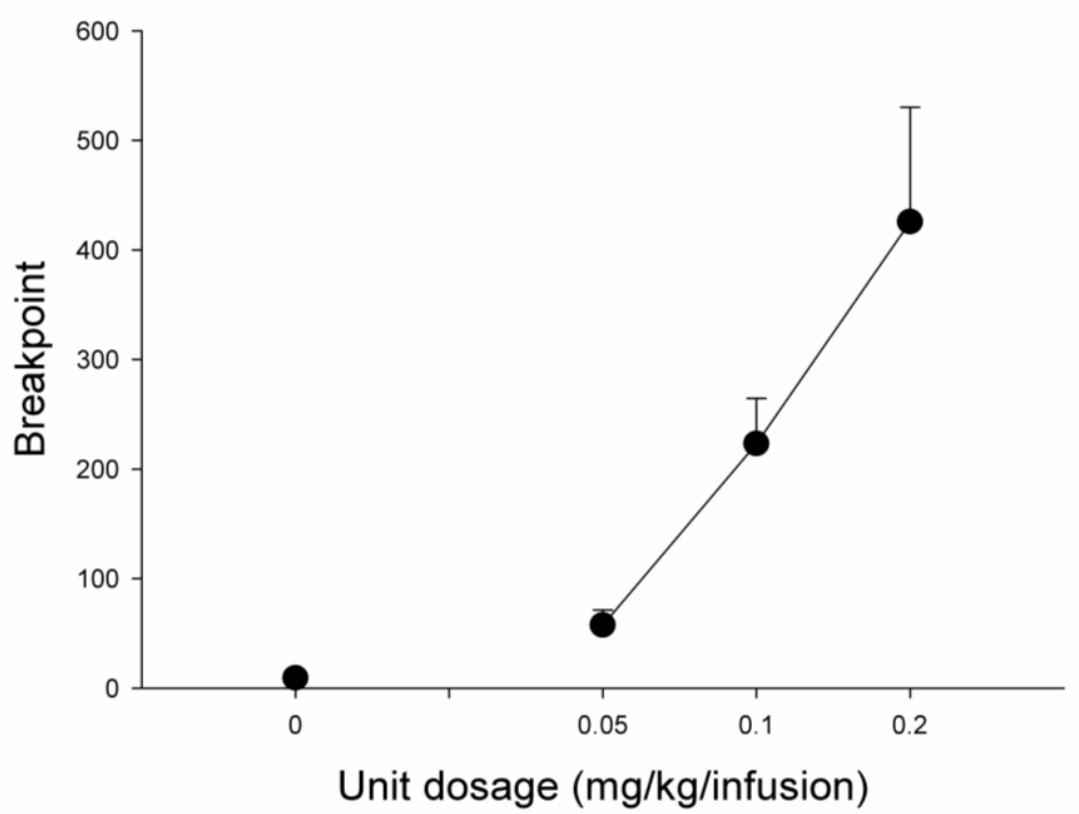

Figure 3. Sample graph of mean (+SEM) BPs reinforced by drug self-administration according to a PR schedule of reinforcement as a function of unit drug dose.

The $\mathrm{BP}$, as a reliable measure of reinforcing efficacy and incentive motivation, depends partly on the criterion time frame for completing each ratio. As stated above, BPs are measured independent of response rates, but they are still dependent on the allotted time frame for completion of each ratio within the paradigm. This means that if the criterion ratio period is too short relative to the inter-injection interval produced by the current unit dose of self-administered drug, 
the BP may be biased toward lower values and vice versa (Markou, et al., 1993). Indeed, some studies have reported declining BPs at higher unit doses of MA, generating an inverted U-shape dose-response curve similar to those generated under FR schedules of reinforcement. For instance, PR responding was supported by four doses of MA (0.01, 0.03, 0.1, $0.3 \mathrm{mg} / \mathrm{kg} /$ infusion), with BPs increasing and reaching a peak when reinforced by the three lower doses, and decreasing when responding was reinforced by $0.3 \mathrm{mg} / \mathrm{kg} /$ infusion (Clemens, et al., 2006). It is possible that some higher doses of psychostimulants may be less positively reinforcing due to the unconditioned aversive subjective effects of the drug, experienced as being 'over the top', thereby accounting for lower BPs at higher doses (Griffiths, et al., 1979; Roberts, et al., 2007; Stafford, et al., 1998; Wilson, et al., 1971). The reduction in BPs produced by the highest dose of self-administrated MA reported by Clemens et al. (2006), however, may reflect paradigmatic temporal limitations and should not be interpreted as a decrease in the reinforcing efficacy of MA, at first glance. It is possible that the criterion time for completion of each ratio may be responsible for lower BPs at higher doses, as the introduction of an inter-trial interval time-out (TO) period can eliminate the descending limb of a dose-response function on a PR schedule of reinforcement (Stafford, et al., 1998). For instance, cocaine selfadministration on a PR schedule of reinforcement generated a biphasic doseresponse curve (inverted U-shape), with lower BPs produced at higher doses (Rowlett, Massey, Kleven, \& Woolverton, 1996). When longer inter-trial TO periods were introduced, however, dose-dependent responding remained a positive linear function of dose, reaching an asymptote and higher BPs at higher doses. This suggests that the reduction in BPs seen at higher doses of MA self-administration (Clemens, et al., 2006) may be more representative of the temporal limits imposed on completing each ratio, rather than a reduction in the reinforcing efficacy of MA at higher doses.

The use of both FR and PR schedules of reinforcement to investigate the neurobiological mechanisms of drug-produced positive reinforcement can provide important complementary findings. Often, experimental manipulations, such as dose variations and pharmacological pre-treatments, produce inversely related effects on each schedule of reinforcement (McGregor \& Roberts, 1993; Richardson \& Roberts, 1996). That is, as the self-administered unit dose of drug increases, BPs increase on a PR schedule of reinforcement; while response rates on an FR schedule of 
reinforcement decrease. Indeed, self-administration of three doses of GBR 12909 $(0.375,0.75,1.5 \mathrm{mg} / \mathrm{kg} /$ infusion), a DAT inhibitor, on a FR1 schedule of reinforcement generated a dose-response curve with the lowest dose maintaining the highest rate of responding, and the highest dose producing the slowest response rates (i.e. the descending limb of the dose-response curve; Roberts, 1993). The same doses of GBR 12909 produced a positive linear dose-response function characterized by increasing BPs with increases in unit dose. These findings were compared to cocaine self-administration on the same schedules of reinforcement. The response rates for GBR 12909 on the FR1 schedule of reinforcement were approximately three times slower than for equivalent doses of cocaine, while BPs reinforced by equivalent doses of GBR 12909 and cocaine were comparable (Roberts, 1993). This indicated that GBR 12909 has a longer duration of action compared to cocaine, but possesses a similar reinforcing efficacy. Thus, using several unit doses of selfadministered drug and both paradigms to investigate changes in reinforcing efficacy as a function of unit dose can increase the convergent validity of any inferences made following experimental manipulations on either schedule.

FR and PR schedules of reinforcement can be effectively used to complement conclusions based on findings from either paradigm. Changes on one schedule of reinforcement, however, may not necessarily be reflected in the other, emphasizing the importance of examining the impact of experimental manipulations on BP as well as rate of responding on a FR schedule of reinforcement. Indeed, systemic pre-treatment with SB-277011A (12.0, $24.0 \mathrm{mg} / \mathrm{kg})$, a DA antagonist, significantly reduced BPs reinforced by MA self-administration $(0.05$ $\mathrm{mg} / \mathrm{kg} /$ infusion), but did not significantly affect MA self-administration reinforced by the same dose according to a FR2 schedule of reinforcement (Higley, et al., 2011). A study only examining MA reinforced responding on an FR schedule of reinforcement may have erroneously concluded that SB-277011A pre-treatment did not affect MA self-administration behaviour. Complementing this finding with a reduction in MA reinforced BPs on a PR schedule of reinforcement following the same experimental manipulation provides a clearer picture of the dopaminergic mechanisms mediating the positively reinforcing effects of MA.

Paradigmatic differences may account for the discrepancy in results between FR and PR schedules of reinforcement. For instance, animals responding on a PR schedule of reinforcement may receive more training, and therefore greater exposure 
to the self-administered drug. This may produce neuroadaptations rendering PR selfadministration behaviour more, or less, susceptible to pharmacological manipulations. Further, the two schedules of reinforcement produced different levels of MA intake potentially influencing the effects of SB-277011A: total MA intake under the FR2 schedule of reinforcement averaged $2.5 \mathrm{mg}$, whereas total MA intake under the PR schedule averaged only $0.80 \mathrm{mg}$ (Higley, et al., 2011). The greater levels of MA exposure during self-administration reinforced by an FR2 schedule may therefore have reduced the pharmacological efficacy of SB-277011A at DA receptors, rendering this manipulation ineffective. Thus, the use of both an FR and PR schedule of reinforcement provided a clearer picture of the dopaminergic mechanisms involved in MA self-administration.

Further clarification of differential effects on PR and FR schedules of reinforcement may be achieved with pharmacological manipulations administered locally into brain tissue. Because anatomical specificity is not possible with systemic injections, a less selective behavioural effect may be produced. In contrast, specific intracerebral manipulations make possible the differentiation between neuroanatomical substrates and their contribution to drug self-administration behaviour. Indeed, local intra-striatal pre-treatment with SCH 23390 (0-2.0 $\mu \mathrm{g} / 0.5$ $\mu l /$ side), a DA antagonist, produced a dose-dependent increase in response rates maintained by IV cocaine self-administration $(1.5 \mathrm{mg} / \mathrm{kg} /$ infusion) reinforced on an FR1 schedule, suggesting a decrease in cocaine's reinforcing efficacy (McGregor \& Roberts, 1995). The same manipulation had no effect, however, on BPs reinforced with the same dose of cocaine under a PR schedule of reinforcement, suggesting this manipulation does not affect cocaine's reinforcing efficacy. Similarly, intraamygdala injections of SCH 23390 (0.1-2.0 $\mu \mathrm{g} /$ injection) produced significant increases in rate of responding supported by cocaine $(1.5 \mathrm{mg} / \mathrm{kg} /$ infusion $)$ under an FR1 schedule of reinforcement, while the same pharmacological pre-treatment had little effect on cocaine reinforced BPs under a PR schedule of reinforcement (McGregor \& Roberts, 1993). The above findings suggest that BPs on a PR schedule of reinforcement and rate of responding on a FR schedule of reinforcement provide different information about drug self-administration behaviour. Not only may each dependent variable reflect distinct aspects of drug-produced positive reinforcement, but experimental manipulations may produce significant effects on one measure and not the other. Thus, investigating the influence of experimental manipulations on 
BPs under a PR schedule of reinforcement and response rates under FR schedules of reinforcement may provide crucial insight into drug self-administration behaviour not attainable with either schedule alone (Arnold \& Roberts, 1997; Gardner, 2000; Griffiths, et al., 1979).

The PR schedule of reinforcement is not without limitations. Possibly the most problematic is that only a single data point is provided from an entire selfadministration session. When studying the effect of pharmacological manipulation on drug self-administration behaviour it is imperative that the effects of each drug coincide within the same timeframe. Thus, the effects of a drug with a short half-life that would be expected to decrease BPs might wear off before the effect on BP is observed. While some paradigms yield a stream of data during a session (e.g. assessing drug-produced locomotor activity in an open field), allowing the time course of drug action to be continuously assessed, PR schedules only provide a single measure of the incentive motivation for a further drug infusion - the BP. Ceiling and floor effects may also limit the sensitivity of a PR schedule of reinforcement. Because many drugs of abuse, possessing different mechanisms of action, show similar maximal BPs it may be difficult to detect differences between them. With that in mind, high BPs reflect a high incentive motivation and great reinforcing efficacy, potentially indicating a high abuse liability, so false positive determinations may not pose a problem. False negatives, however, may arise and because pharmacological history and route of administration can affect reinforcing efficacy (Carlson, 2004; Julien, 2001), using BPs to assess abuse liability requires that the drug is administered under optimal reinforcing conditions. Responding under a PR schedule of reinforcement also requires more training, and studies using this method typically take longer to complete than, for example, studies using FR schedules of reinforcement. This increases the risk of losing catheter patency in experimental animals, making reliable and valid data collection more difficult.

Responding on a PR schedule of reinforcement represents an excellent method of determining changes in reinforcing efficacy of a self-administered drug, and provides a measure of incentive motivation for further drug-taking. Because the BP is a measure of reinforcing efficacy independent of rate of responding, any interpretational ambiguities inherent in changes in response rate on a FR schedule of reinforcement are ameliorated, and the two measures can be effectively used to complement inferences based on findings from either paradigm (Arnold \& Roberts, 
1997; Griffiths, et al., 1979; Richardson \& Roberts, 1996; Stafford, et al., 1998). Thus, experimental manipulations on both a PR and FR schedule of reinforcement can tell us much more about drug self-administration than studying behaviour on either schedule alone, greatly increasing the convergent validity of any findings. These schedules of reinforcement have been extensively used to further our understanding of the neurobiological basis of drug self-administration.

\section{$4 \quad$ Neural mechanisms of drug self-administration}

Self-administration of drugs of abuse is based on operant theory, stipulating that a reinforcing stimulus will increase the likelihood of the behaviour that produced it recurring in the future. By demonstrating that laboratory animals voluntarily initiated and maintained self-administration of a number of drugs, across several drug classes, Deneau and colleagues (1969) established that drugs that are abused by humans are also positively reinforcing to laboratory animals. Since then, a great deal of research has been devoted to establishing the neurobiological mechanisms of drug abuse, its initiation, maintenance, relapse, and consequences. Drugs of abuse come from a variety of origins (synthetic, organic) and diverse classes (stimulants, depressants, narcotic analgesics), suggesting that their primary pharmacodynamics may vary substantially. These diverse primary mechanisms do not, however, preclude the possibility of a final common mechanism of drugmediated positive reinforcement. Various drugs of abuse have neither chemical nor pharmacological commonality, begging the questions of what these compounds have in common, and what distinguishes them from compounds that are not selfadministered? It appears that the answer to both of these questions resides in the universal ability of drugs of abuse to activate the dopaminergic system. There is a vast literature on other neurotransmitter mechanisms involved in the regulation of drug self-administration (e.g. 5-HT, NE, GABA, glutamate; Adinoff, 2004; Di Chiara, 1999; Di Chiara, et al., 2004; Gardner, 2000; Olive, 2005; Smith, Co, Freeman, \& Lane, 1982; Sulzer, 2001), but review of these is beyond the scope of this thesis. For this reason, the common neurochemical substrates of drug produced positive reinforcement and mechanisms of drug self-administration investigated within this thesis will be discussed in greater detail. 
Despite accounting for less than $1 \%$ of the brain's neuronal population (Marsden, 2006), DA systems have been suggested as the primary mechanism of reinforcement for drugs of abuse, including MA (Adinoff, 2004; Feltenstein \& See, 2008; Ikemoto, 2007; Sulzer, 2001; Wise \& Bozarth, 1987; Wise \& Rompre, 1989; Yokel \& Wise, 1976). With very few exceptions, drugs self-administered by humans are self-administered by animals (Deneau, et al., 1969; Schuster \& Thompson, 1969; Weeks, 1962; Wilson, et al., 1971; Yokel \& Pickens, 1973) and drugs that are voluntarily self-administered by laboratory animals enhance brain reinforcement mechanisms in the mesolimbic DA system (Gardner, 2000; Marsden, 2006; Wise \& Rompre, 1989). These commonalities of self-administered drugs form the basis for the theory that drug self-administration is positively reinforcing through its effects on dopaminergic mechanisms. Indeed, in 1989 it was already suggested that "cocaine and amphetamine are rewarding because of their actions in dopaminergic synapses" (p. 214, Wise \& Rompre, 1989). For instance, MA and many psychostimulants' primary action is to increase synaptic levels of the monoamines by blocking, and/or reversing membrane transporters (Camp, Browman, \& Robinson, 1994; Fischer \& Cho, 1979; Julien, 2001; Sulzer, 2001; Sulzer, et al., 2005). The increase in extracellular levels of DA has been suggested to mediate the primary positive reinforcing properties of these drugs, while NE and 5-HT can modulate these effects (Di Chiara \& Bassareo, 2007; Di Chiara, et al., 2004; Di Chiara \& Imperato, 1988; Munzar, Baumann, Shoaib, \& Goldberg, 1999). Opiates (e.g. morphine, heroin) primary mechanism is to stimulate the opioid receptors, particularly $\mu(\mathrm{mu})$ receptors (Cruickshank \& Dyer, 2009; Julien, 2001; Martin, 1983). Stimulation of these opioid receptors inhibits GABA neurons in the VTA, preventing the tonic inhibition of dopaminergic neurons, thereby producing increased DA release in terminal regions (Martin, 1983; Shalev, Grimm, \& Shaham, 2002). Thus, diverse primary mechanisms of drugs of abuse may converge on a common secondary mechanism of increased DA activity.

An increased level of DA activity appears to be the essential common neurochemical substrate of drug self-administration. Support for the idea that drugs of abuse increase dopaminergic neurotransmission has been obtained using brain microdialysis following experimenter-administered injections measuring extracellular synaptic DA concentrations in the NAc and caudate nucleus (dorsal Str) as an index of DA transmission in freely moving rats (Di Chiara \& Imperato, 1988). 
The results showed that drugs of abuse from diverse pharmacological classes, such as amphetamine, cocaine, morphine, methadone, ethanol and nicotine, increased extracellular DA concentrations in both regions, but more so in the NAc. These findings were extended to differentiate between the shell and core of the NAc in the increased extracellular DA release produced by various drugs of abuse. Brain microdialysis in freely moving rats showed that doses of cocaine, morphine and amphetamine that sustain self-administration behaviour increased synaptic DA concentrations preferentially in the shell, as opposed to the core of the NAc (Pontieri, Tanda, \& Di Chiara, 1995). This effect is also seen following exposure to MA. For example, acute MA injections in both drug-naïve rats, and challenge injections in MA pre-treated rats, produced a significant increase in DA overflow in the Str (Hamamura, et al., 1991; Shuto, et al., 2006; Y. Zhang, Loonam, \& Noailles, 2001). Thus, what drugs of abuse with diverse primary mechanisms may have in common is the ability to mediate the positive reinforcement necessary for their selfadministration through a common final mechanism - the dopaminergic system.

With few exceptions, what most drugs of abuse have in common, and what differentiates them from drugs that are not abused, is the ability to activate the dopaminergic system. Specifically, drugs with aversive properties, such as $\kappa$ opioid agonists (U-50.488, bremazocine, tifluadom; Mucha \& Herz, 1985) reduced DA release, as measured by brain dialysis in freely moving rats (Di Chiara \& Imperato, 1988). Further, drugs that are not abused by humans, such as imipramine (antidepressant), atropine (muscranic antagonist) and diphenhydramine (antihistamine), did not alter synaptic DA concentrations in the Str or NAc (Di Chiara \& Imperato, 1988). Thus, the common denominator among most drugs of abuse appears to be the ability to increase dopaminergic neurotransmission. Together with the above findings, this provides in vivo evidence supporting the idea that the ability of drugs of abuse to reinforce the behaviour that delivers them is somehow linked with their ability to increase synaptic DA concentrations in the mesolimbic DA system.

A wealth of preclinical research has suggested a crucial role for DA in the drug-produced positive reinforcement supporting self-administration behaviour. For instance, rodents self-administered both the dextro $(d)$ and levo $(l)$ isomers of MA and amphetamine, but lower doses of the $d$-, compared to $l$-isomer, were able to maintain self-administration behaviour (Yokel \& Pickens, 1973). This indicates a 
central dopaminergic mechanism may be the proximal cause of MA reinforcement, as each isomer is equipotent in autonomic, peripheral actions, but the $d$-isomer is more potent in central dopaminergic mechanisms (Wise \& Rompre, 1989). Research examining DA levels during MA self-administration is somewhat lacking, however, increased DA levels were reported when cocaine was self-administered on a FR1 schedule of reinforcement. Specifically, extracellular DA levels in the NAc were elevated by $200-800 \%$ during cocaine $(2.0 \mathrm{mg} / \mathrm{kg} / \mathrm{infusion})$ self-administration, fluctuating within this range between operant responses (Wise, et al., 1995). There were larger increases in DA levels and a slower rate of responding following infusions of higher unit doses. These findings lend further support to the hypothesis that rate of responding for cocaine self-administration is titrated across doses for stable hourly drug intake, and that falling DA levels trigger successive responding for further cocaine infusions (Wise, et al., 1995).

Selective pharmacological manipulation of dopaminergic systems producing altered patterns of operant behaviour has revealed further support for a role of DA in drug self-administration. Altered patterns of drug self-administration following selective pharmacological manipulation of the DA system have been reported for a wide range of addictive drugs, both psychostimulants and opiates (Bari \& Pierce, 2005; Barrett, Miller, Dohrmann, \& Caine, 2004; Bergman, Kamien, \& Spealman, 1990; Botly, Burton, Rizos, \& Fletcher, 2008; Brennan, Lake, et al., 2007; Caine \& Koob, 1994; Caine, Negus, Mello, \& Bergman, 1999; Daniela, Brennan, Gittings, Hely, \& Schenk, 2004; David, Durkin, \& Cazala, 2002; Higley, et al., 2011; Hubner \& Moreton, 1991; McGregor \& Roberts, 1995; McGregor \& Roberts, 1993; Munzar, et al., 1999; Phillips, Robbins, \& Everitt, 1994; Pulvirenti, Balducci, Piercy, \& Koob, 1998; Wilson \& Schuster, 1972; Yokel \& Wise, 1976; D. Zhang, et al., 2010). Specifically, pharmacological manipulations that decrease the reinforcing efficacy of the self-administered drug may increase response rates on an FR schedule of reinforcement in a compensatory manner, and decrease BPs on a PR schedule of reinforcement (similar to decreasing the unit dose of self-administered drug). This effect was demonstrated with chlorpromazine pre-treatment on drug selfadministration reinforced under a FR schedule. Chlorpromazine, the prototypical antipsychotic agent and DA antagonist, increased FR responding for cocaine, pipradrol, phenmetrazine, $d$-amphetamine, and methylphenidate in rhesus monkeys (Wilson \& Schuster, 1972). Amphetamine self-administration reinforced according 
to a PR schedule of reinforcement was also altered following selective manipulation of the DA system. Pre-treatment with the DA antagonists, raclopride, and eticlopride but not the 5-HT antagonist, ritanserin (Fletcher, 1998), dose-dependently reduced BPs maintained by amphetamine self-administration (Fletcher, 1998; Izzo, Orsini, Koob, \& Pulvirenti, 2001). Further, pre-treatment with terguride, a partial DA agonist that functions as an agonist in conditions of low DA receptor occupancy, and an antagonist in conditions of high DA levels (such as during amphetamine selfadministration), significantly decreased amphetamine reinforced BPs on a PR schedule of reinforcement (Izzo, et al., 2001).

Pharmacological manipulations that increase the reinforcing efficacy of the self-administered drug, on the other hand, may reduce response rates on a FR schedule of reinforcement and increase BPs (analogous to increasing the unit dose of self-administered drug on the descending limb of the dose-response curve). For instance, pre-treatment with the indirect DA agonist, phentermine, decreased MA self-administration reinforced according to a FR schedule (Munzar, et al., 1999), and pre-treatment with other DA agonists, such as amphetamine, quinelorane, 7hydroxy-dipropylaminotetralin (7-OH-DPAT) and GBR 12909, produced dosedependent left-ward shifts in the dose-response function for cocaine selfadministration (Barrett, et al., 2004; Caine, et al., 1999). Further, the indirect DA agonists, phentermine and GBR 12909, both generalized to cocaine and MA in a drug-discrimination task (Holtzman, 2001; Munzar, et al., 1999), and produced dose-dependent reductions in self-administration of either drug, analogous to a leftward shift in the dose-response curve (Munzar, et al., 1999; Tella, 1995). The leftward shift in the dose-response function suggests an increase in cocaine's reinforcing efficacy. The idea of DA mediated positive reinforcement is further supported by the finding that GBR 12909 promoted voluntary initiation and maintenance of self-administration behaviour, under both FR and PR schedules (Roberts, 1993). Thus, selectively blocking DA reuptake is positively reinforcing. These findings suggest that self-administration behaviour in laboratory animals is supported by indirect DA agonists and is selectively altered by pharmacological manipulation of the DA system.

Pharmacological manipulation that eliminates the reinforcing properties of the self-administered drug should produce a sequential change in responding characterized by an initial compensatory increase, followed by a decrease in 
responding, before ceasing entirely. This typical extinction of responding was demonstrated following DA receptor blockade during both MA and amphetamine self-administration. Amphetamine self-administration was increased following pretreatment with low doses of the DA antagonists pimozide and (+)-butaclamol, and altered in a biphasic manner following high doses - response rates initially increased before decreasing, and then ceasing entirely (Yokel \& Wise, 1976). Similar findings were produced on MA self-administration which was attenuated by pre-treatment with the VMAT2 inhibitors lobeline, and lobelane (Harrod, et al., 2001; Neugebauer, 2007; Nickell, et al., 2010). Lobeline and lobelane prevent the inhibition of MAO by MA and bind to the VMAT2, expelling DA into the presynaptic cytosol where it is metabolised to DOPAC, thereby preventing the reverse transport and increased release induced by MA (Dwoskin \& Crooks, 2002; Nickell, et al., 2010; Wilhelm, Johnson, Eshleman, \& Janowsky, 2008). These findings represent classic behavioural extinction in the face of a lack of reinforcement, as even with increased blood levels of amphetamine, responding ceased following full DA blockade (Yokel \& Wise, 1976), and the lobeline-induced decrease in responding for MA was not surmounted by increasing the unit dose of MA (Harrod, et al., 2001). This suggests a non-specific rate decreasing effect, and a non-competitive mechanism of action for lobeline (Dwoskin \& Crooks, 2002; Teng, Crooks, Sonsalla, \& Dwoskin, 1997). Importantly, lobelane pre-treatment did not affect sucrose self-administration suggesting the decrease in responding produced by DA antagonism was selective to drug-produced positive reinforcement (Neugebauer, 2007). Further, while lobeline initially decreased responding for sucrose reinforcement, tolerance developed to this effect with repeated administrations, but the decrease in responding maintained by MA persisted (Harrod, et al., 2001), suggesting a more drug-selective effect. The support for enhanced DA neurotransmission in the reinforcing and addictive properties of drugs of abuse can be summarized by stating that an increase in extracellular DA levels follows exposure to all of the classically addictive drugs: DA receptor partial blockade increases drug self-administration, with cessation at full blockade; and drug self-administration is inhibited following DA synthesis blockade, or decreased catecholamine release by VMAT2 inhibition (Sulzer, 2001).

Because many psychostimulants, including MA, produce substantial increases in 5-HT and NE neurotransmission (Julien, 2001; Raiteri, et al., 1979; Ricaurte, et al., 1980; Rothman, et al., 2001; Sulzer, et al., 2005), these monoamines 
are also implicated in the neurobiological mechanisms of drug self-administration. Self-administration studies have demonstrated a limited role of NE in mediating psychostimulant reinforcement or supporting operant responding (Weinshenker \& Schroeder, 2007), while serotonergic mechanisms may serve a modulatory role (Munzar, et al., 1999; Wise \& Rompre, 1989). For instance, NE and 5-HT agonists did not significantly alter cocaine self-administration (Tella, 1995). Reducing NE synaptic transmission by systemic injection of the $\alpha-\mathrm{NE}$ antagonist, phentolamine, or $\beta$-NE antagonist, $l$-propranolol, failed to alter amphetamine self-administration (Yokel \& Wise, 1976). The DAT inhibitor, GBR 12909, supported voluntary initiation and maintenance of self-administration behaviour, under both FR and PR schedules (Roberts, 1993), indicating that blocking DA reuptake is positively reinforcing, while self-administration of NE reuptake inhibitors/agonists is not typically supported (Tessel \& Woods, 1975; Wee, et al., 2006). These findings suggest that DA mediates the discriminative and reinforcing properties of psychostimulant self-administration, while NE systems may serve a limited role in this behaviour (Holtzman, 2001; Munzar, et al., 1999; Rothman \& Baumann, 2006).

5-HT mechanisms, on the other hand, can modulate psychostimulant selfadministration (Miszkiel, Filip, \& Przegaliński, 2011; Müller, Carey, Huston, \& De Souza Silva, 2007; Munzar, et al., 1999). A study of the relative reinforcing strength of MA to MDMA and its isomers found that MA maintained higher BPs on a PR schedule of reinforcement than the racemic, positive, or negative isomers of MDMA, with a reinforcing efficacy relationship of $\mathrm{MA}>(+)-\mathrm{MDMA}>( \pm) \mathrm{MDMA}>(-) \mathrm{MDMA}$ (Wang \& Woolverton, 2007). This relationship relates to each compound's monoamine releasing potency and the ratio of DA over 5-HT release is identical to the reinforcing efficacy of these compounds, as demonstrated by higher BPs reached. At initial exposure, MA releases roughly 30-fold more DA than 5-HT, whereas MDMA is about sevenfold more potent in releasing 5-HT than DA (Rothman, et al., 2001). Thus, the compound with the highest DA/5-HT ratio, MA, was the most effective reinforcer, compared to the weakest reinforcer, (-)MDMA, with the lowest DA/5-HT ratio (Rothman \& Baumann, 2006; Wang \& Woolverton, 2007). MDMA pre-treatment in rats that self-administered MA reinforced according to a PR schedule of reinforcement produced dose independent reductions of BPs, interpreted as a reduction in MA's reinforcing efficacy by the inhibitory effects of MDMAinduced 5-HT release on dopaminergic mechanisms (Clemens, et al., 2006). 
Systemic injections of the 5-HT agonist, fenfluramine, attenuated MA selfadministration on an FR schedule (Munzar, et al., 1999). Depleting forebrain 5-HT levels by injecting the neurotoxin 5,7-dihydroxy-tryptamine (5,7-DHT) into either the medial forebrain bundle, or amygdala, significantly increased BPs maintained by cocaine self-administration (Loh \& Roberts, 1990). BPs maintained by amphetamine self-administration were reduced by the mixed DA and 5-HT antagonist risperidone, and the D2-like antagonist raclopride, while the 5-HT antagonist, ritanserin, did not significantly alter BPs (Fletcher, 1998). Further, in contrast to DAT inhibitors, SERT inhibitors do not support self-administration behaviour (Howell \& Byrd, 1995; Tessel \& Woods, 1975), indicating they do not mediate positive reinforcement and possess little potential for abuse. Taken together, these findings suggest that the reinforcing properties of psychostimulants supporting their self-administration are mediated primarily through DA pathways, and that 5-HT systems may play a modulating role in the mediation of psychostimulant self-administration and reinforcement (Holtzman, 2001; Munzar, et al., 1999; Rothman \& Baumann, 2006).

\subsection{Dopamine receptors and drug self-administration}

Drugs of abuse increase extracellular levels of DA and pharmacological manipulation of the DA system produced significant changes in drug selfadministration patterns on both FR and PR schedules of reinforcement. The effects of DA stimulation and pharmacological manipulations of the DA system, however, depend on the specific type and the localisation of the receptor the neurotransmitter/drug binds to (Carlson, 2004; Missale, Nash, Robinson, Jaber, \& Caron, 1998). Following the discovery that DA receptors could either stimulate or inhibit adenylyl cyclase (AC) activity, five DA receptor subtypes, which fall into one of two receptor categories, have been characterized (Missale, et al., 1998). DA D1-like receptors (D1 and D5) are positively coupled to AC, stimulating 3',5'-cyclic monophosphate (cAMP) generation; whereas DA D2-like receptors either have no effect on $\mathrm{AC}$, or are negatively coupled to $\mathrm{AC}$, inhibiting $\mathrm{cAMP}$ formation (Kebabian, Blanchet, \& Bedard, 1995; Self, 1998, 2004b; Self \& Nestler, 1998). D1like receptors are more widely distributed, and can be located both pre- and postsynaptically with postsynaptic localization more frequently observed (Levey, et al., 1993; Missale, et al., 1998). D1-like receptors have also shown extrasynaptic localization (Gonon, 1997; Smiley, Levey, Ciliax, \& Goldman-Rakic, 1994). D2- 
like receptors show frequent localization as both autoreceptors and postsynaptic receptors (Carlson, 2004; Levey, et al., 1993; Missale, et al., 1998). For instance, one of the most prominent regulators of DA neuron activity is the D2 autoreceptor. These receptors are found in the dendrites, soma and terminal buttons of DA neurons, and their localisation determines their effects. Specifically, activation of dendritic and somatic D2 autoreceptors produces hyperpolarization and decreases neural firing (Carlson, 2004). Activation of terminal button D2 autoreceptors, on the other hand, decreases the activity of an enzyme in the chain of DA biosynthesis, tyrosine hydroxylase $(\mathrm{TH})$, thus decreasing DA production and finally its release (Carlson, 2004). Thus, stimulation of D2 autoreceptors may down-regulate DA neuron activity, maintaining their activity within a stable rate of firing (Grace, 2002), whereas stimulation of postsynaptic D2 receptors may increase DA-mediated behaviours, such as drug self-administration (Missale, et al., 1998; Wise, Murray, \& Bozarth, 1990).

In terms of drug self-administration, these receptors often mediate similar effects (Missale, et al., 1998; Self \& Nestler, 1998; Waddington, et al., 1995). For instance, both receptor subtypes appear to mediate drug-produced positive reinforcement, as selective receptor agonists at both subtypes can substitute for cocaine and are self-administered (Self, Belluzzi, Kossuth, \& Stein, 1996; Weed \& Woolverton, 1995; Wise, et al., 1990). For instance, squirrel monkeys trained to lever press for IV cocaine reinforcement, maintained FR, and second-order fixedinterval (FI) responding when infusions were replaced with agonists selective for the D1 (SKF 82958), and D2 (quinpirole) receptors, respectively (Grech, Spealman, \& Bergman, 1996). Rats also self-administered agonists selective for each receptor, as SKF 82958 (Self, Belluzzi, et al., 1996), and the D2 agonist, bromocriptine (Wise, et al., 1990) both support IV self-administration. In addition, equimolar mixtures of the D1 agonist SKF 38393 and quinpirole, at concentrations that alone did not support intra-cranial self-administration, were self-infused at significant rates directly into the NAc shell (Ikemoto, Glazier, Murphy, \& McBride, 1997). This suggests that concurrent activation of D1 and D2 receptors in the NAc shell had a synergistic effect on DA mediated reinforcement processes. Thus, selective activation of D1 and/or D2 receptor subtypes produced positive reinforcement capable of maintaining self-administration behaviour. 
Changes in drug self-administration patterns following pre-treatment with D1-like agonists and antagonists, further supports the role of DA and this receptor subtype in drug self-administration. For instance, pre-treatment with D1-like antagonists increased cocaine self-administration in rats on FR (Barrett, et al., 2004; Brennan, Lake, et al., 2007; Caine, Heinrichs, Coffin, \& Koob, 1995; Caine \& Koob, 1994; Chang, Sawyer, Lee, \& Woodward, 1994; Hubner \& Moreton, 1991; McGregor \& Roberts, 1995; McGregor \& Roberts, 1993; Ranaldi \& Wise, 2001), and second-order fixed interval (FI) schedules of reinforcement (Bergman, et al., 1990). Intra-NAc self-administration of a D1 and D2 agonist combination (SKF 38393 + quinpirole) on a FR schedule of reinforcement was abolished following D1like receptor antagonist pre-treatment, indicating a full blockade of positive reinforcement (Ikemoto, et al., 1997). Pre-treatment with D1-like antagonists reduced BPs reinforced by cocaine (Hubner \& Moreton, 1991; McGregor \& Roberts, 1995; McGregor \& Roberts, 1993; Ranaldi \& Wise, 2001), methylphenidate (Botly, et al., 2008) and 'speedball' infusions (cocaine and heroin combination; Cornish, Lontos, Clemens, \& McGregor, 2005) on a PR schedule of reinforcement. D1-like antagonist pre-treatment increased $N$-benzylpiperazine (BZP; Brennan, Lake, et al., 2007), MDMA (Daniela, et al., 2004), and methylphenidate (Botly, et al., 2008) selfadministration. Pre-treatment with the selective D1 agonists SKF 82958 and SKF 77434 decreased cocaine self-administration (Barrett, et al., 2004; Caine, et al., 1999). These findings suggest a critical role for DA D1-like receptor mechanisms in the self-administration of drugs of abuse. The effects of manipulating D1-like receptor mechanisms on measures of MA self-administration, however, have yet to be explored.

Selective pharmacological manipulation of D2-like receptor mechanisms also altered drug self-administration patterns. Pre-treatment with D2-like antagonists produced an increase in cocaine self-administration in rats (Barrett, et al., 2004; Bergman, et al., 1990; Caine \& Koob, 1994; Hubner \& Moreton, 1991), or extinction of the operant behaviour following higher doses (Chang, et al., 1994). The D2-like antagonist, eticlopride, also produced a dose-dependent increase in cocaine self-administration in mice (Caine, et al., 2002). Self-administration of other drugs of abuse are also affected by selective D2-like antagonism, as pre-treatment with eticlopride produced an increase in methylphenidate (Botly, et al., 2008), and MDMA self-administration (Brennan, Carati, Lea, Fitzmaurice, \& Schenk, 2009) in 
rats. In addition, the direct D2 agonists, quinelorane and 7-hydroxydipropylaminotetralin (7-OH-DPAT), both decreased cocaine self-administration in a manner similar to increasing the unit dose of cocaine (Barrett, et al., 2004; Caine, et al., 1999). Finally, intra-NAc self-administration of a D1 and D2 agonist combination (SKF 38393 + quinpirole) was attenuated following pre-treatment with the D2-like antagonist, sulpiride (Ikemoto, et al., 1997).

Changes in the reinforcing efficacy of several drugs of abuse have also been suggested by selective manipulation of D2-like receptor mechanisms. Indeed, pretreatment with eticlopride reduced BPs on a PR schedule reinforced by cocaine (Bari \& Pierce, 2005) and methylphenidate (Botly, et al., 2008) infusions. The D2-like antagonist raclopride, and mixed D2-like and 5-HT2 receptor antagonist, risperidone, both reduced BPs produced by amphetamine (Fletcher, 1998). D2 partial agonists reduced heroin (D. Zhang, et al., 2010), amphetamine (Izzo, et al., 2001), and cocaine (Pulvirenti, et al., 1998) reinforced BPs. Taken together, the above findings indicate a crucial involvement of DA D1- and D2-like receptor mechanisms in mediating the reinforcing properties of drugs of abuse and maintaining drug selfadministration behaviour. D2-like mechanisms have been implicated in the incentive motivation and reinforcing efficacy of MA, as BPs reinforced by MA on a PR schedule of reinforcement were attenuated following pre-treatment with the DA D3 receptor antagonist, SB-277011A (Higley, et al., 2011), and the partial D2 agonist, aripiprazole (Wee, et al., 2007). Further, CJB090, a partial agonist at the D3 receptor, reduced extended ( $6 \mathrm{hr}$ ) access MA self-administration reinforced according to both an FR and PR schedule of reinforcement, while the D3 full antagonist, PG01037, reduced MA reinforced BPs on a PR schedule (Orio, Wee, Newman, Pulvirenti, \& Koob, 2010). The contribution of D2-like mechanisms to the maintenance of MA self-administration, however, has not yet been further explored.

\section{$5 \quad$ Relapse and reinstatement of drug-seeking}

One of the greatest obstacles to the successful treatment of drug dependence is the high rate of relapse to drug abuse, even after prolonged periods of abstinence (APA, 2000; Degenhardt, et al., 2008; Eisinger, Wodarski, \& Ferguson, 2009; Elkashef, 2008; Yahyavi-Firouz-Abadi \& See, 2009). Relapse to drug-seeking and eventually drug-taking behaviour can be studied in the reinstatement paradigm of 
drug self-administration (de Wit \& Stewart, 1981). Reinstatement of drug-seeking is a measure that has been used to elucidate the neurobiological mechanisms responsible for the characteristic cyclical pattern of abstinence, drug craving and relapse to drug abuse. Following acquisition of drug self-administration, responding is extinguished by replacing drug infusions with vehicle (behavioural extinction), until a criterion level of responding is produced (e.g. < $20 \%$ of baseline number of responses). Extinguished responding can subsequently be reinstated by stimuli that have previously been reported to induce drug-craving and precipitate relapse in humans, namely: exposure to the drug or a drug with similar properties (drugpriming; de Wit \& Stewart, 1981), stress (Stewart, 2000), and/or drug-associated cues (Shaham \& Stewart, 1996). There are two main dependent variables in reinstatement of drug-seeking: vehicle-reinforced responding on the previously drug-associated lever (active lever), interpreted as reflecting the magnitude of reinstated drug-seeking; and responses on the inactive lever, interpreted to reflect non-specific behavioural activation.

The great intuitive appeal of the reinstatement paradigm is that it possesses substantial criterion, or face validity, as the variables that have produced reinstatement of drug-seeking behaviour in laboratory animals have also been reported to induce subjective reports of drug craving, and provoke relapse in humans (Epstein, Preston, Stewart, \& Shaham, 2006; Katz \& Higgins, 2003). A great number of studies have demonstrated that re-exposure to the drug of abuse, or a drug with similar subjective properties; exposure to drug-associated cues; and/or stress can evoke drug-seeking behaviour in laboratory animals and drug craving and relapse in humans (Epstein \& Preston, 2003; Epstein, et al., 2006; Kalivas \& McFarland, 2003; Shaham, Shalev, Lu, de Wit, \& Stewart, 2003; Shalev, et al., 2002; Stewart, 2000; Volkow, et al., 2006). It should be pointed out that drug craving, however, cannot be reduced to an observable event, but rather can be conceived of as a motivational state in a causal sequential link with behaviour culminating in drug-seeking, and drug-taking. It is only the behavioural response that can be measured in animal studies of relapse and reinstatement of drug-seeking, whereas the subjective description of the cause for the behaviour can only be inferred (Epstein, et al., 2006; Katz \& Higgins, 2003; Self \& Nestler, 1998). Thus, the reinstatement paradigm of animal drug self-administration can be effectively used to explicitly measure extinguished drug-seeking behaviour, and we may infer that 
craving may motivate some aspects of this behaviour, but drug-craving itself cannot be measured in animals.

Each method of reinstating extinguished drug-seeking has been reliably demonstrated in laboratory animals trained to self-administer MA. MA-seeking behaviour was reinstated by priming injections of MA, presentation of discrete cues associated with self-administered MA delivery (Anggadiredja, Sakimura, Hiranita, \& Yamamoto, 2004; Hiranita, Nawata, Sakimura, Anggadiredja, \& Yamamoto, 2006; Hiranita, Yamamoto, \& Nawata, 2010; Moffett \& Goeders, 2007; Rogers, et al., 2008; Shelton \& Beardsley, 2008), and stress (Shepard, Bossert, Liu, \& Shaham, 2004). For instance, rats were trained to self-administer MA and subsequently underwent extinction training for 12 days either with, or without response contingent drug-paired cues (Shelton \& Beardsley, 2008). Reinstatement of extinguished MAseeking behaviour was then induced by a priming injection of MA (1 mg/kg, IP) with and without contingent presentation of previously drug-paired cues. Further, response-contingent presentation of previously MA-paired cues alone, without MApriming, reinstated MA-seeking behaviour (Shelton \& Beardsley, 2008). Extinguished MA-seeking was also reinstated by the experience of stress, induced through intermittent electric foot-shock, and pharmacologically, by systemic injections of the anxiogenic drug, yohimbine (Shepard, et al., 2004).

A theoretical issue that has been raised regarding the reinstatement paradigm as a model of drug-seeking relapse to drug-taking is that the contingencies surrounding the reinstatement test itself differ between the animal model and human relapse. In drug-primed tests for reinstatement, the priming injections are given noncontingently by the experimenter, whereas in cue-induced reinstatement, presentation of the discrete drug-associated cues typically depends on the operant behaviour of the animal (Shalev, et al., 2002). Conversely, relapse to drug abuse in humans typically involves contingent exposure to the drug, and/or noncontingent exposure to drug-related cues. For the human abuser, drug re-exposure is more often a consequence of a 'lapse', rather than an initiator of relapse (Katz \& Higgins, 2003). Thus, the drug priming-injection that precedes reinstatement of drug-seeking behaviour in the animal model is dissimilar from the self-imposed 'lapse' that may precede full relapse in human users. Despite the discrepancy in the contingency of drug- and cue-exposure, the functional behaviour of drug-priming and drug cueinduced reinstatement of drug-seeking in laboratory animals remain similar to the 
behaviours of relapse in human drug abusers - that is, time spent obtaining (drugseeking) and taking the drug.

Reinstatement of drug-seeking can be studied within-session, betweensession, or with a between-within-session variation of the procedure, depicted in table 1. Within-session reinstatement methods conduct tests of drug selfadministration, extinction training, and finally a test for reinstatement of drugseeking all within the same day (e.g. de Wit \& Stewart, 1981). Between-session reinstatement studies conduct drug self-administration, extinction training, and reinstatement tests during different daily sessions, facilitating the investigation of the neurobiological mechanisms involved in relapse when the animal is free of selfadministered drug (e.g. Schenk, Gittings, \& Colussi-Mas, 2011; Schenk, Hely, Gittings, Lake, \& Daniela, 2008). Between-within-session reinstatement tests allow animals to self-administer a drug across several days followed by extinction training and reinstatement testing on the same day (e.g. Shaham, et al., 2003). These methods may employ different time frames for reinstatement testing, but they all measure the same behaviour - reinstatement of extinguished responding. For the purposes of this thesis, the between-session reinstatement method was used as animals are free of self-administered drug at the time of testing, and testing is not typically affected by the aversive symptoms of acute drug withdrawal (Shalev, et al., 2002).

\begin{tabular}{|c|c|c|c|}
\hline & $\begin{array}{l}\text { Drug self- } \\
\text { administration }\end{array}$ & $\frac{\text { Extinction }}{\text { training }}$ & $\frac{\text { Reinstatement }}{\text { test }}$ \\
\hline Within & Day 1 & Day 1 & Day 1 \\
\hline
\end{tabular}

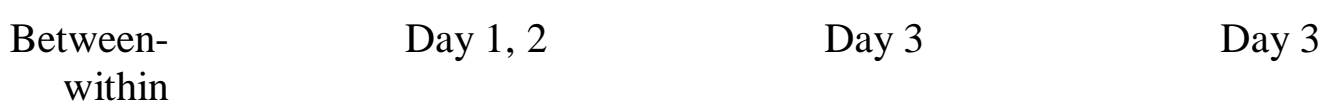
Between
Day 1,2
Day 3,4
Day 5

Table 1. A depiction of sample timelines of within-session, between-within-session, and between-session reinstatement procedures.

Some researchers, but by no means all, have suggested that the drug withdrawal period that may accompany drug abstinence may play a role in the mediation of relapse to drug abuse. According to this idea, the alleviation of the aversive subjective state of drug withdrawal (negative reinforcement) is thought to be one of the key reasons for compulsive drug use and repetitive relapse among drug-dependent individuals (Koob, 2005, 2006; Koob \& Le Moal, 2001; Shaham, et 
al., 2003). Studies on changes in the magnitude of the drug-seeking response as a function of the withdrawal period (i.e. the interval elapsed since a drug was last selfadministered), however, have revealed findings counterintuitive of the negative reinforcement theory. Reports indicate that reinstatement of drug-seeking behaviour in animals was greatest at time points well beyond the acute withdrawal period, and that the least reinstatement was observed following only one day of withdrawal, when the aversive effects of acute drug withdrawal would be expected to be their most pronounced (Epstein \& Preston, 2003; Shaham, et al., 2003). For instance, compared to one day of abstinence, cocaine-seeking behaviour in rats was highest at one month of abstinence (Tran-Nguyen, et al., 1998), and extracellular DA levels were more elevated in the amygdala (Tran-Nguyen, et al., 1998), and NAc (Hollander \& Carelli, 2007). This suggests that while withdrawal symptoms may contribute to further drug-seeking and -taking through negative reinforcement in humans users, drug withdrawal syndromes are not critical in relapse to drug-seeking in laboratory animals, as measured by the reinstatement paradigm. Rather, the duration of the withdrawal period following cessation of drug self-administration exerts profound quantitative effects on the magnitude of reinstatement, and extracellular DA levels.

Reinstatement of extinguished drug-seeking behaviour represents an excellent paradigm for the investigation of the neurobiological mechanisms involved in relapse to drug-seeking and ultimately drug-taking. The paradigm has criterion, or face validity in the sense that the same variables that have been reported to induce subjective drug-craving and promote relapse to drug-seeking and drug-taking in abstinent human drug users also induce reinstatement of extinguished drug-seeking behaviour in laboratory animals (Katz \& Higgins, 2003; Shaham, et al., 2003; Shalev, et al., 2002). These variables include exposure to the previously selfadministered drug, or a drug with similar interoceptive properties, exposure to environmental stimuli that have become associated with drug-taking behaviour, and experiencing stress. Reinstatement of extinguished MA-seeking behaviour has been demonstrated with each method. Preclinical studies on reinstatement of drug-seeking have confirmed clinical observations - that drug priming, or a single exposure to the relevant drug of abuse (e.g. the one draw on a MA pipe that precipitates a full binge), is the most potent trigger of relapse and drug-seeking behaviour (Adinoff, 2004). For the purposes of investigating the factors involved in reinstatement of drug-seeking 
behaviour and which neural mechanisms are implicated, the reinstatement model arguably represents the most valid animal model of drug abuse relapse (Kalivas \& McFarland, 2003). Studying reinstatement of drug-seeking behaviour using the between session paradigm can provide important information on the functional state of the neurobiological mechanisms involved in relapse at a time that may be beyond any effects of drug withdrawal, or short-term (e.g. 48 hrs) changes in those mechanisms, and when the animal is free of self-administered drug (Shalev, et al., 2002). Thus, the reinstatement paradigm can be used to investigate one of the greatest obstacles to successful treatment of drug abuse and dependence.

\subsection{Dopamine and drug-seeking}

Relapse to drug abuse in humans, and reinstatement of drug-seeking in laboratory animals can be produced by presentation of drug-associated cues, exposure to stress, and by exposure to the drug itself (Katz \& Higgins, 2003; Self, 1998; Self \& Nestler, 1998; Shaham, et al., 2003; Shalev, et al., 2002). Findings from studies using animal models of drug-seeking suggest relapse triggered by stress, drug-cues, and drug-priming are mediated by distinct neurochemical circuits, but converge on a final common pathway through dopaminergic systems (Kalivas \& McFarland, 2003; Self, 1998; Stewart, 1984). For instance, during tests of drugseeking controlled by cocaine-associated cues, there were marked increases in dialysate DA levels in dorsal striatum (Ito, Dalley, Robbins, \& Everitt, 2002), NAc, and amygdala (Weiss, et al., 2000). Correspondingly, cue-controlled drug-seeking under a second-order schedule was attenuated by local infusions of the non-selective DA antagonist, $\alpha$-flupenthixol, into the dorsal Str (Vanderschuren, Di Ciano, \& Everitt, 2005). In the reinstatement paradigm, drug-seeking triggered by drugassociated cues was reduced by inhibition of midbrain (SN and VTA), and dorsal Str DA regions by local infusions of GABA agonists (baclofen + muscimol; See, Elliott, $\&$ Feltenstein, 2007), and by unilateral lesion of the DA rich NAc core and infusion of $\alpha$-flupenthixol into the contralateral dorsal Str (Belin \& Everitt, 2008). Further evidence for the involvement of the dopaminergic system in drug cue-produced reinstatement has been suggested by altered drug-seeking behaviour following pretreatment with DA agonists and antagonists selective for either D1- and/or D2-like receptors. For instance, cocaine-seeking reinstated by response-contingent presentation of a light + tone stimulus complex previously paired with cocaine 
infusions was attenuated by pre-treatment with the direct D1 agonist, SKF 81297, and D1-like antagonist, SCH 23390 (Alleweireldt, Weber, Kirschner, Bullock, \& Neisewander, 2002). Drug-cue produced heroin-seeking was also attenuated by both systemic and intra-NAc shell injections of SCH 23390 (Bossert, Poles, Ihbey, Koya, \& Shaham, 2007). In addition, nicotine-seeking triggered by drug-associated cues was attenuated by pre-treatment with $\mathrm{SCH} 23390$, and the D2-like antagonist, eticlopride (Liu, et al., 2010). Together, these findings indicate an important role for DA and D1- and D2-like receptor mechanisms in reinstatement of drug-seeking behaviour triggered by stimuli previously associated with drug-taking.

Stress-induced relapse has been most often demonstrated in laboratory animals using a brief period of mild intermittent electric foot-shock, but has also been induced pharmacologically. For instance, previously extinguished MA-seeking behaviour was produced in rats by five minutes of intermittent $(0.5 \mathrm{sec} \mathrm{ON}$, mean OFF period of $40 \mathrm{sec}$, range 10-70 sec), inescapable, electric foot-shock $(0.4,0.6$ $\mathrm{mA}$ ), delivered through a scrambler to the grid floor of the operant chambers (Shepard, et al., 2004). Reinstatement of MA- (Shepard, et al., 2004) and cocaineseeking (Anker \& Carroll, 2010) was also produced following systemic injections of the anxiogenic drug, yohimbine. Stress-induced reinstatement is mediated through dissimilar neurobiological pathways to drug- and cue-induced reinstatement (Kalivas \& McFarland, 2003; Stewart, 2000), but some studies have suggested the dopaminergic system may modulate this behaviour (Self, 1998; Shalev, et al., 2002). Indeed, pre-treatment with $\alpha$-flupenthixol attenuated foot-shock induced heroinseeking (Shaham \& Stewart, 1996). The PFC has also been implicated as infusions of SCH 23390 directly into the prelimbic cortex or obitofrontal cortex blocked footshock-induced reinstatement of cocaine-seeking (Capriles, 2003). Conversely, selective antagonism of D1- or D2-like receptor mechanisms by systemic pretreatment with SCH 23390 or raclopride, had no significant effect on foot-shock induced reinstatement of heroin-seeking, but attenuated drug-primed reinstatement of heroin-seeking behaviour (Shaham \& Stewart, 1996). Together, these findings indicate a secondary/indirect role of DA in foot-shock stress-induced reinstatement of drug-seeking (Shalev, et al., 2002).

Drugs of abuse, across the pharmacological classes, have different primary sites of action, and psychostimulants increase synaptic levels of all the monoamines (DA, 5-HT, NE), but drug-primed reinstatement of drug-seeking appears to be 
primarily mediated through dopaminergic mechanisms (Self, 1998, 2004b; Self \& Nestler, 1998; Shaham, et al., 2003; Stewart, 1983, 2000). For instance, cocaine is a non-selective biogenic monoamine transporter inhibitor that binds with higher affinity to the SERT, than DAT or NET (Julien, 2001; Ritz, Cone, \& Kuhar, 1990). Following extinguished cocaine self-administration, priming-injections of the DAT inhibitor, GBR 12909, but not the NET inhibitor, nisoxetine, nor the SERT inhibitor, fluoxetine, dose-dependently reinstated cocaine-seeking behaviour (H. D. Schmidt \& Pierce, 2006). Infusions of morphine directly into the VTA reinstated previously extinguished cocaine- and heroin-seeking behaviour (Stewart, 1984), suggesting that DA activity in this region may facilitate the priming effects of both psychostimulants and opiates (Stewart, 1983). Further, intra-NAc shell injections of the DA antagonist, $\alpha$-flupenthixol, attenuated GBR 12909-induced reinstatement of cocaine-seeking, suggesting a primary dopaminergic contribution to this behaviour (H. D. Schmidt \& Pierce, 2006). Extinguished drug-seeking in rats previously trained to selfadminister heroin was also reinstated by priming-injections of GBR 12909 (De Vries, Schoffelmeer, Binnekade, Raas $\varnothing$, \& Vanderschuren, 2002), and DA receptor antagonists attenuated drug-seeking produced by heroin priming-injections (Shaham $\&$ Stewart, 1996). Together, these findings suggest that an increase in extracellular DA, specifically in the NAc shell, is both necessary and sufficient for drug-induced relapse to psychostimulant- and opiate-seeking behaviour.

Further evidence for the role of DA in drug-primed reinstatement of drugseeking comes from studies showing significant effects on this behaviour following selective pharmacological manipulation of D1- and D2-like receptor mechanisms. Although direct D1 and D2 agonists are both self-administered (Grech, et al., 1996; Ikemoto, et al., 1997; Self, Belluzzi, et al., 1996; Weed \& Woolverton, 1995; Wise, et al., 1990), and pharmacological manipulations of D1- and D2-like receptor mechanisms produced similar effects on measures of drug-taking reinforced according to FR and PR schedules of reinforcement (Bari \& Pierce, 2005; Barrett, et al., 2004; Brennan, et al., 2009; Brennan, Lake, et al., 2007; Caine, et al., 1995; Caine \& Koob, 1994; Caine, et al., 1999; Daniela, et al., 2004), they may mediate qualitatively different aspects of drug-seeking (responding maintained in the absence of drug-reinforcement). Studies using the self-administration reinstatement model of relapse (de Wit \& Stewart, 1981) have indicated that D1-like receptors may play a facilitative, or permissive, role in mediating the effects of D2-like receptor 
stimulation on relapse to drug-seeking (Bergman, et al., 1990; Missale, et al., 1998; Phillips, et al., 1994; Self, Barnhart, Lehman, \& Nestler, 1996). For instance, selective stimulation of D2 receptors has reinstated cocaine- (De Vries, et al., 2002; De Vries, Schoffelmeer, Binnekade, \& Vanderschuren, 1999; Dias, Lachize, Boilet, Huitelec, \& Cador, 2004; Khroyan, Barrett-Larimore, Rowlett, \& Spealman, 2000; Self, Barnhart, et al., 1996), MDMA- (Schenk, et al., 2011) and heroin-seeking (De Vries, et al., 2002; De Vries, et al., 1999; Wise, et al., 1990). Conversely, selective stimulation of D1 receptors failed to reinstate these behaviours (De Vries, et al., 1999; Dias, et al., 2004; Khroyan, et al., 2000; Schenk, et al., 2011; Self, Barnhart, et al., 1996), suggesting that $\mathrm{D} 2$ receptors play a primary role in inducing drug-seeking behaviour (Self, 2004b). Blockade of either D1- or D2-like receptor mechanisms, on the other hand, attenuated reinstatement of cocaine- (Alleweireldt, et al., 2002; Anderson, Bari, \& Pierce, 2003; Capriles, 2003; Green \& Schenk, 2002; Khroyan, et al., 2000; Khroyan, Platt, Rowlett, \& Spealman, 2003; Milivojevič, Krisch, Sket, \& Živin, 2004; Schenk \& Gittings, 2003), heroin- (Bossert, et al., 2007; Shaham \& Stewart, 1996), MDMA- (Schenk, et al., 2011), and nicotine-seeking behaviour (Liu, et al., 2010). Thus, because drug-seeking was produced by direct D2 agonists, but not by direct D1 agonists, and was attenuated by both selective D1- and D2-like antagonists, these findings suggest that stimulation of D1-like receptors alone is insufficient for reinstatement of drug-seeking, but may be necessary in enabling D2like receptor mediation of this behaviour. That is, D1-like receptor activation may play a permissive role in the expression of drug-seeking behaviour mediated by stimulation of D2-like receptor mechanisms.

Reinstatement of extinguished MA self-administration in laboratory animals has been reliably demonstrated, and the dopaminergic system has been implicated in this behaviour, but compared to other drugs of abuse there is a relative paucity of studies on dopaminergic mechanisms of reinstatement of MA-seeking behaviour. Following extinguished MA self-administration $(0.05 \mathrm{mg} / \mathrm{kg} /$ infusion $)$ reinforced according to a FR2 schedule, MA-seeking was produced by a priming injection of MA (1 mg/kg, IP; Higley, et al., 2011). Pre-treatment with the DA D3 receptor antagonist, SB-277011A, significantly attenuated MA-seeking, suggesting that this behaviour can be altered through selective pharmacological manipulation of the dopaminergic system. Further, reinstatement of MA-seeking produced by MAassociated cues, and MA-priming injections was attenuated following pre-treatment 
with (-)-BPAP, a catecholaminergic enhancer, providing additional support for the idea of a dopaminergic mechanism of reinstatement of MA-seeking behaviour (Hiranita, et al., 2010). A wealth of research with other drugs of abuse has suggested crucial roles for both D1- and D2-like receptor mechanisms in the reinstatement of drug-seeking behaviour. The contributions of DA D1- and D2-like receptor mechanisms underlying reinstatement of MA-seeking, however, have yet to be further investigated.

\section{Summary and the present study}

Drug self-administration is an excellent tool for furthering our understanding of the neurobiological mechanisms of drug abuse and dependence. Selfadministration paradigms and the schedule of reinforcement they employ must be tailored, however, to accurately reflect the aspect of drug abuse that is under investigation - no single paradigm can answer all questions relevant to the study of drug abuse and dependence. Much research into the neurobiological mechanisms of drug self-administration has suggested that the dopaminergic system is crucial to the initiation of drug use, the transition to compulsive abuse and dependence, and to the maintaining cycle of drug-craving and relapse to drug abuse. The dopaminergic system also appears especially vulnerable to the neurotoxic effects of MA exposure, producing potentially debilitating functional deficits (Barr, et al., 2006; Camp, et al., 1994; Fischer \& Cho, 1979; Fischman \& Schuster, 1974; Friedman, et al., 1998; NIDA, 2006; Tziortzis, Mahoney Iii, Kalechstein, Newton, \& La Garza Ii, 2011; Volkow, et al., 2001). The majority of research into the potentially neurotoxic effects of MA, however, has utilized non-contingent exposure protocols, limiting the interpretation of MA-produced neuroadaptations following voluntary selfadministration. Studying the effects of repeated exposure to self-administered MA in laboratory animals can ameliorate these contingency issues.

Like many other drugs of abuse, MA facilitates DA neurotransmission in terminal brain regions that comprise the mesocorticolimbic and nigrostriatal DA pathways (Barr, et al., 2006; Baumann, et al., 2002; Camp, et al., 1994; Cruickshank \& Dyer, 2009; Julien, 2001). A great deal of research supports the idea that this effect is critical to drug self-administration. For example, DAT inhibitors, and direct D1 and D2 receptor agonists are self-administered (Grech, et al., 1996; Roberts, 
1993; Weed \& Woolverton, 1995), and pharmacological blockade of the DAT decreased self-administration (Barrett, et al., 2004). Further, pre-treatment with D1and D2-like antagonists reduced BPs produced by drugs of abuse, and increased self-administration under FR schedules of reinforcement (Anderson, et al., 2003; Barrett, et al., 2004; Caine \& Koob, 1994; Caine, et al., 2002). This suggests that DA D1- and D2-like receptor mechanisms mediate the preliminary positive reinforcement necessary for the initiation and maintenance of drug selfadministration, the reinforcing efficacy of the self-administered drug and incentive motivation for further drug-taking.

As an animal model of relapse to drug abuse, the reinstatement model of drug-seeking (de Wit \& Stewart, 1981) has indicated important roles for D1- and D2-like receptor mechanisms in this behaviour. Specifically, selective pharmacological blockade of D1- and D2-like receptors attenuated reinstatement of drug-seeking; and selective stimulation of D2 receptors reinstated drug-seeking behaviour (Self, 1998, 2004b; Self \& Nestler, 1998; Shaham, et al., 2003; Shaham \& Stewart, 1996; Stewart, 2000).

Considering the epidemiologic status of MA abuse and dependence here in New Zealand, but also worldwide, there is a relative paucity of studies investigating the neurobiological mechanisms of MA self-administration. The vast majority of laboratory studies on the effect of pharmacological manipulation of DA receptors on psychostimulant self-administration have focused on cocaine as the drug of interest. Dopaminergic mechanisms of MA self-administration and reinstatement of MAseeking, specifically those mediated by D1- and D2-like receptor mechanisms, are less clear, and have yet to be thoroughly investigated. This warrants further analysis, as although cocaine and MA induce similar behavioural responses, they evoke different effects at the neurochemical level depending on the brain region studied(Camp, et al., 1994), duration of drug treatment (Y. Zhang, et al., 2001), and produce different subjective and cardiovascular effects in humans (Newton, et al., 2005). In addition, divergent neuroadaptations following self-administration of cocaine vs. MA suggest that the effects of D1- and D2-like receptor manipulations might not extrapolate to MA self-administration. Specifically, rats exposed to selfadministered MA showed extensive reductions in D1- and D2-like receptor binding densities in the VTA and SN (Stefanski, et al., 1999). These neuroadaptations were not evident following exposure to self-administered cocaine under similar protocols 
(Stefanski, et al., 2007). This raises the possibility that pharmacological manipulation of D1- and D2-like receptor mechanisms may have different effects on self-administration of MA.

The aim of the present study was, therefore, to investigate the neurobiological mechanisms of MA self-administration, focusing on the dopaminergic system, and to determine the extent of any functional D1- and/or D2like receptor mechanism neuroadaptations at short ( $24 \mathrm{hr}$ ) or longer ( $72 \mathrm{hr}-7$ day) withdrawal times. The following set of experiments aims to firstly determine the effect of exposure to chronically self-administered MA on dopaminergic and serotonergic markers of nerve terminal degeneration in the Str and FC, using high performance liquid chromatography (HPLC) with neurochemical detection. Any potential for neurochemical recovery will be assessed following a short ( $24 \mathrm{hr}$ ) and long ( 7 day) withdrawal period. In addition, the effect of contingency on these measures will be determined using a yoked model of self-administration. Because any potential DA reductions following MA self-administration may affect the response to manipulations of DA D1- and D2-like receptor mechanisms, the findings from this study will provide an indication of the functional status of the DA system during maintenance and reinstatement of MA self-administration in later experiments.

Secondly, as neuroadaptations may take place throughout the dopaminergic system over the course of chronic drug abuse, it may be expected that D1- and D2like receptor mechanisms make differential contributions to MA self-administration throughout the course of drug abuse. Assessing different aspects of MA selfadministration with different schedules of reinforcement (FR vs. PR) and at different stages of MA abuse (maintenance vs. relapse) may provide a functional indication of the involvement of D1- and D2-like receptor mechanisms in this behaviour. To achieve this, the contribution of DA D1- vs. D2-like receptor mechanisms to the maintenance of MA self-administration will be determined under a schedule of continuous reinforcement (FR1) by pharmacological manipulation of these receptors. Further, D1- and D2-like receptor mediated mechanisms of the incentive motivation for MA reinforcement, and changes in the reinforcing efficacy of MA, will be determined by changes in BPs on a PR schedule of reinforcement following pretreatment with D1- and D2-like antagonists. Using both a FR and PR schedule of reinforcement will provide greater convergent validity indicating that behavioural 
differences between manipulations likely reflect neuroadaptations as a function of MA self-administration, as opposed to procedural differences between each schedule. Assessing the effects of acute pre-treatment with a DA D1- or D2-like receptor antagonist on MA self-administration reinforced according to a FR and PR schedule will provide a functional assay of these receptor mechanisms complementing any neurochemical findings at the short withdrawal period obtained in the first experiment.

Finally, the contribution of DA D1- and D2-like receptor mechanisms to relapse to MA abuse will be investigated through pharmacological manipulation of these receptor systems and the consequent effects on reinstatement of extinguished MA-seeking. Because the state of the dopaminergic system may be different during drug exposure as opposed to following abstinence, it may be expected that DA D1and D2-like receptor mechanisms would contribute differentially to MA selfadministration at different stages (maintenance vs. relapse). Using the betweensession method of the reinstatement paradigm will allow a test of the contribution of these receptor mechanisms to this behaviour at a time when the animal is free of self-administered drug, providing further functional evaluation of these receptor mechanisms in the light of any neurochemical findings at the long withdrawal period from the first experiment.

The first experiment examining changes in neurotransmitter tissue content as a function of MA self-administration at short (24 hrs) and long (7 days) withdrawal times will provide an important indication of the general neurochemical status of the dopaminergic system at these times. The findings from this experiment may then be complemented by functional assays of the dopaminergic system at different withdrawal times by acute selective pharmacological manipulation of D1- and D2like receptor mechanisms and the effects of this on MA self-administration, and on MA-seeking behaviour. The neurochemical findings at the short withdrawal time in the first study will be complemented by a functional assessment of DA D1 - and D2like receptor mechanisms in MA self-administration at the same withdrawal time on an FR1 and PR schedule of reinforcement. The neurochemical findings at the long withdrawal time in the first study can then be complemented by a functional assessment of DA D1- and D2-like receptor mechanisms in the reinstatement of extinguished MA-seeking behaviour in the reinstatement paradigm. 


\section{General Methods}

\section{Subjects}

Adult male Sprague-Dawley rats weighing approximately 300-350 g rats were used for all self-administration experiments. All rats were bred in the vivarium at Victoria University of Wellington and were housed in groups of four from weaning until they reached appropriate weights. They were then individually housed in standard polycarbonate cages in a temperature $\left(21^{\circ} \mathrm{C}\right)$ - and humidity $(77 \%)$ controlled room, and handled each day, three days prior to surgery. The colony was maintained on a 12-hr light/dark cycle (lights on at 0700), and all testing was conducted during the light portion of the cycle, beginning at 1400. Food and water were available ad libitum, except during testing. The colony was accredited by the Office of Laboratory Animal Welfare (OLAW), and principles of laboratory animal care were adhered to (NIH publication NO. 85-23, rev. 1985). All experimental procedures were approved by the Victoria University Animal Ethics Committee.

\section{Apparatus}

Self-administration training and testing were carried out in operant chambers (Med Associates, ENV 001, Georgia, Vermont, USA), enclosed in sound attenuating boxes. Each chamber had a plexiglass door, rear wall and ceiling; the lever mounted, and opposite wall were stainless steel; while the floor was grilled bars over a woodchip catch tray. The ceiling of each chamber had a hole in the centre to allow the passing of microbore tubing connected to a $20 \mathrm{ml}$ syringe in an automatic pump (Razel Model A, 1 rpm motor, Georgia, Vermont, USA) into the chamber. Each chamber contained two levers and a stimulus light located above the right lever. Depression of the right lever ("active lever") resulted in a $0.1 \mathrm{ml}$ infusion delivered over $12 \mathrm{sec}$. Coincident with drug delivery was the illumination of the stimulus light located above the active lever, for the duration of the infusion. Depressions of the left lever ("inactive lever") were recorded, but were without programmed consequence. Drug delivery and data recording were controlled by an interfaced microcomputer utilizing Med Associates software. 


\section{Procedures}

\subsection{Surgery}

Catheters were constructed in-house using silastic tubing $(0.51 \mathrm{~mm}$ [inner diameter] x $0.94 \mathrm{~mm}$ [outer diameter]) and a steel tip crafted from a 22 gauge needle. Silicon was applied to the join between tubing and steel tip, and to the proximal end of the catheter to be inserted into the vein to protect the tubing from any corrosion by superglue. Rats were implanted with chronic indwelling jugular vein catheters under deep anaesthesia produced by separate injections of xylazine ( 5 $\mathrm{mg} / \mathrm{kg}, \mathrm{IP}$ ) and ketamine (75 mg/kg, IP) given in their home cage. Anaesthesia was ascertained by foot pinch. Rats were then moved to the surgical suite, injected with the anti-inflammatory Carprofen ${ }^{\circledR}(5 \mathrm{mg} / \mathrm{kg}, \mathrm{SC}$, Pfizer Animal Health), their chests were shaved and cleaned with $85 \%$ ethanol, and iodine, and Lacrilube ${ }^{\circledR}$ was applied to their eyes to prevent drying out. A small incision was made in the chest and the right external jugular vein was isolated and tied off using surgical thread. Another incision was made on the top of the head from between the eyes to between the ears, the underlying tissue was removed using a scalpel blade to expose the skull, a topical antibiotic was applied, and the catheter was passed subcutaneously from this incision to the chest incision. A small cut was made in the jugular vein; the catheter was inserted and secured in place using surgical thread and super glue. The chest incision was closed with super glue and a topical antibiotic was applied. The rat was then placed in a stereotaxic and the steel-tipped distal end of the catheter was secured to the skull using acrylic dental cement adhered to four small jeweller's screws imbedded in the skull. Following surgery, Hartmann's sodium lactate solution ( $6 \mathrm{ml}$ each side of hindquarters, SC) was administered to replenish bodily fluids and electrolytes. Rats were then placed in a warmed recovery tray until the anaesthesia wore off, and finally returned to their home cage.

Carprofen® $(5 \mathrm{mg} / \mathrm{kg}, \mathrm{SC})$ was administered on each of the two days following surgery. During each of the five days comprising the recovery period, rats were weighed, and catheters were flushed with $0.1 \mathrm{ml}$ of sterile $0.9 \%$ saline solution, containing heparin $(30 \mathrm{IU} / \mathrm{ml})$ and penicillin $\mathrm{G}$ potassium $(250,000 \mathrm{IU} / \mathrm{ml})$. At the start of the testing period, and every seven days thereafter, catheter patency was tested with a $0.1 \mathrm{ml}$ infusion of sodium pentobarbital $(5.0 \mathrm{mg} / \mathrm{kg}, \mathrm{IV})$. Catheter patency was confirmed by loss of the righting reflex within $5 \mathrm{sec}$. If catheter patency 
was lost, a second catheter was inserted into the left jugular vein as described above and the rat was given a minimum of three days recovery time, during which no testing was conducted.

\subsection{Daily self-administration}

Training and experimental testing was conducted five days per week, Monday to Friday, during two hour daily sessions. At the start of each experimental session, the catheters were flushed with $0.2 \mathrm{ml}$ of the $30 \mathrm{UI} / \mathrm{ml}$ heparin/penicillin solution. Immediately prior to each experimental session, rats were transferred from their home cages to the humidity $(55 \%)$ and temperature $\left(21^{\circ} \mathrm{C}\right)$ controlled experimental room in a woodchip littered plastic carry box. The steel tip of each catheter was attached to the microbore tubing in the chamber connected to a $20 \mathrm{ml}$ syringe in an automatic pump. The session began with an experimenter-delivered response on the active lever to clear the line of the heparin solution. Following each infusion there was a $30 \mathrm{sec}$ timeout (TO) period during which responding on the active lever was without consequence. At the end of each self-administration session, catheters were again flushed with $0.2 \mathrm{ml}$ of the $30 \mathrm{UI} / \mathrm{ml}$ heparin/penicillin solution, followed by $0.1 \mathrm{ml}$ of a $30 \mathrm{IU} / \mathrm{ml}$ heparinised saline $0.9 \%$ solution containing $8000 \mathrm{IU} / \mathrm{ml}$ streptokinase to prevent the formation of fibroids. Rats were then returned to their home cages in the woodchip littered plastic carry box.

\subsection{Acquisition of self-administration}

During training, MA $(0.1 \mathrm{mg} / \mathrm{kg} /$ infusion $)$ was self-administered according to a FR1 schedule of reinforcement during daily two hour sessions. This dose was based on our preliminary work and that of other studies showing reliable selfadministration (Shelton \& Beardsley, 2008; Stefanski, et al., 1999). Selfadministration was considered acquired when 1) there were at least 10 infusions earned per session; 2) the ratio of active lever to inactive lever presses during a session was at least $2: 1$; and 3) these criteria were met for at least three consecutive days with less than $20 \%$ variation in number of active lever responses.

\section{Drugs}

MA hydrochloride (ESR, Porirua, New Zealand) was dissolved in a $0.9 \%$ saline solution and administered via IP injection at a volume of $1 \mathrm{ml} / \mathrm{kg}$, for acute dosing. For self-administration experiments, MA was dissolved in a $0.9 \%$ saline 
solution containing $3 \mathrm{IU} / \mathrm{ml}$ heparin, and IV infusions were delivered in a volume of $0.1 \mathrm{ml}$. All drug doses refer to the weight of the salt.

\section{Statistical analyses}

All analyses were conducted using the SPSS statistical package v.18, for Microsoft Windows. Specific analyses and experimental sample sizes are detailed in their relevant chapters and on figures. 


\section{Chapter 1: Effects of MA self-administration on monoamine and metabolite tissue concentrations}

\section{Overview}

The aim of Chapter 1 was to investigate the effects of MA exposure on tissue levels of monoamines and metabolites and to determine whether the effects were persistent or transient. This was achieved using a typical experimenter-administered regime as well as a yoked method of self-administration which allowed effects of contingency to be dissociated from effects of experimenter-administered MA.

An experimenter-administered exposure regime that has previously been shown to produce deficits in tissue levels of monoamines and neurotoxic effects (Chapman, Hanson, Kesner, \& Keefe, 2001; Herring, et al., 2008; Quinton \& Yamamoto, 2006) was administered. This treatment regime produced significant decreases in tissue levels of monoamines 24 hours (Pubill, et al., 2003), three days (Herring, et al., 2008), one week (Quinton \& Yamamoto, 2006), three weeks (Chapman, et al., 2001) and seven weeks (Friedman, et al., 1998) following the last MA injection. In the present study, brain levels of monoamines and their metabolites in the Str and FC were measured following a short (24 hour) and longer (seven day) withdrawal period to determine the persistence of any effects. This experiment was intended to verify that the neurotoxic effects produced by this regime persisted throughout the seven day withdrawal period. Thus, this experiment served as a positive control of MA-induced neurotoxicity, rather than as a direct comparison to the effects of self-administered MA-exposure.

The experimenter-administered regime comprising a series of acute, high dose injections administered to drug-naïve rats does not, however, reflect a typical exposure pattern of MA users (Cho, et al., 2001), and several studies have suggested that differential neuroadaptations are produced when the drug is self-administered as opposed to when it is passively administered (Dworkin, et al., 1995; Jacobs, et al., 2003; Stefanski, et al., 2004; Stefanski, et al., 1999; Stefanski, Lee, Yasar, Cadet, \& Goldberg, 2002; Stefanski, et al., 2007). Drug self-administration in laboratory animals has substantial face and construct validity with regard to human drug-taking 
behaviour (Gardner, 2008; Sanchis-Segura \& Spanagel, 2006). Thus, drug selfadministration is a valid means of studying the effects of MA dependence on the brain (Stephens, et al., 2010). Therefore, the effects of self-administered MAexposure on tissue levels of monoamines and metabolites, comparing short and longer withdrawal times were investigated.

A self-administration protocol that has previously shown effects of selfadministered MA on markers of DA neurotransmission was used (Stefanski, et al., 1999). Because long-duration self-administration sessions may more accurately reflect effects of abuse (Kitamura, et al., 2006; Rogers, et al., 2008; Wee, et al., 2007), acquisition of MA self-administration and response-escalation as a marker of dependence was examined in six hour sessions. Self-administration protocols similar to those reported by Stefanski (1999) produced smaller effects on the dopamine system (Schwendt, et al., 2009; Shepard, et al., 2006) than the more typical exposure regimens described above. Specifically, MA self-administration had no effect on DAT mRNA or TH protein levels in the VTA or substantia nigra, and no effect on striatal DA, or prefrontal 5-HT levels (Schwendt, et al., 2009). Self-administered MA produced decreases in DAT densities in both the striatum and prefrontal cortex, but no changes in typical markers of neurotoxicity (e.g. reduced TH levels, glial fibrillary acidic protein, calcium-binding adaptor molecule 1) in these regions (Schwendt, et al., 2009).

These findings are consistent with the idea that MA self-administration does not produce the extensive neurochemical deficits and/or neurotoxicity seen following more stringent experimenter-administered treatment protocols. Tissue levels of monoamines were, however, only assessed at 14 days after the final MA self-administration session (Schwendt, et al., 2009). Most studies examining the effects of MA exposure on tissue levels of monoamines have assessed the effects at earlier withdrawal times, frequently 24 hours after the final MA exposure (Danaceau, et al., 2007; Graham, Noailles, \& Cadet, 2008; O'Neil, et al., 2006; Shepard, et al., 2006; Stefanski, et al., 1999). Assays conducted 24 hours and seven days following the final MA self-administration session will provide a comparison to other studies and may reveal changes in tissue level of monoamines produced selectively by MA self-administration. 


\subsection{Contingency of drug exposure}

High dose or repeated exposure to lower doses of MA produced neurotoxicity and neuroadaptations in dopaminergic and serotonergic neurons (Eisch, Schmued, \& Marshall, 1998; Herring, et al., 2008; O'Dell \& Marshall, 2005; Pubill, et al., 2003; Ricaurte, Guillery, Seiden, Schuster, \& Moore, 1982; Sharma \& Kiyatkin, 2009) and extensive decreases in DA and 5-HT across several brain regions (Quinton \& Yamamoto, 2006). The striatum appears particularly susceptible to MA-induced decreases in DA and its metabolites (Bakit, et al., 1974; Chu, et al., 2008; Friedman, et al., 1998; Thomas, Francescutti-Verbeem, \& Kuhn, 2008; Wagner, et al., 1980). High dose MA administration also reduced 5-HT levels in the frontal cortex and hippocampus of rats (Graham, et al., 2008; Herring, et al., 2008). Because this exposure regime might not reflect effects produced in MA users (Cho, et al., 2001), however, several studies have attempted to more adequately model MA dependence. Methods include chronic exposure via osmotic minipumps (Davidson, et al., 2005), escalating dosing (ED) procedures (Cadet, et al., 2009; Graham, et al., 2008; Segal, et al., 2003), pharmacokinetic modelling (Cho, et al., 2001; Herring, et al., 2008), and IV self-administration (Schwendt, et al., 2009; Shepard, et al., 2006; Stefanski, et al., 2004; Stefanski, et al., 1999; Stefanski, et al., 2002).

ED and pharmacokinetic modelling might provide accurate approximations of dose and pattern of administration by MA users, but both paradigms require noncontingent administration. Several studies have suggested that effects of drug exposure are dependent on the contingency of that exposure, and that differential neuroadaptations are produced when the drug is passively administered, rather than self-administered (Dworkin, et al., 1995; Jacobs, et al., 2003; Stefanski, et al., 2004; Stefanski, et al., 1999; Stefanski, et al., 2002; Stefanski, et al., 2007). For instance, in vivo microdialysis procedures showed greater levels of extracellular DA concentrations in the NAc during cocaine self-administration compared to during yoked cocaine delivery (Hemby, et al., 1997). As measured by in vitro quantitative autoradiography, five weeks of self-administered MA exposure produced a significant reduction in DA D1- (15\% in NAc) and D2-like (34\% in VTA; $31 \%$ in medial SN; $21 \%$ in dorsal $\mathrm{SN}$ ) receptor levels, 24 hours post final MA selfadministration session (Stefanski, et al., 1999). No significant changes were reported on these measures following passive, yoked MA infusions (Stefanski, et al., 1999). Self-administered cocaine did not produce similar changes in DA D2-like receptor 
densities. Rather, there was an increase in D2 receptor mRNA following cocaine self-administration, and a decrease in D2-like receptor densities following passive infusions, further emphasising the differential neurochemical consequences of passive administration versus active self-administration (Stefanski, et al., 2007). The changes in DA receptor density produced by self-administered MA exposure were transient (Stefanski, et al., 1999), and returned to control levels seven days following the final MA self-administration session (Stefanski, et al., 2002). Self-administered MA also failed to produce significant changes in striatal DA or prefrontal cortex 5HT tissue levels when measured following 14 days withdrawal (Schwendt, et al., 2009). This finding is in contrast to the persistent neurochemical changes seen following experimenter-administered treatment regimes that have shown persistent reductions in DA levels at 3 weeks following the final MA treatment (Chapman, et al., 2001; Friedman, et al., 1998).

A 'yoked' procedure of drug self-administration allows one to differentiate neuroadaptations that are produced by the pharmacological effects of the drug alone from those that are involved, additionally, in active drug self-administration behaviour. Animals are grouped into triads; one self-administers the drug (response contingent; $\mathrm{CM}$ ) and the two others receive either drug (yoked MA; YM) or vehicle (yoked vehicle; YV) infusions according to the behaviour of the self-administering subject. Figure 5 illustrates the basic mechanics of yoked drug self-administration.

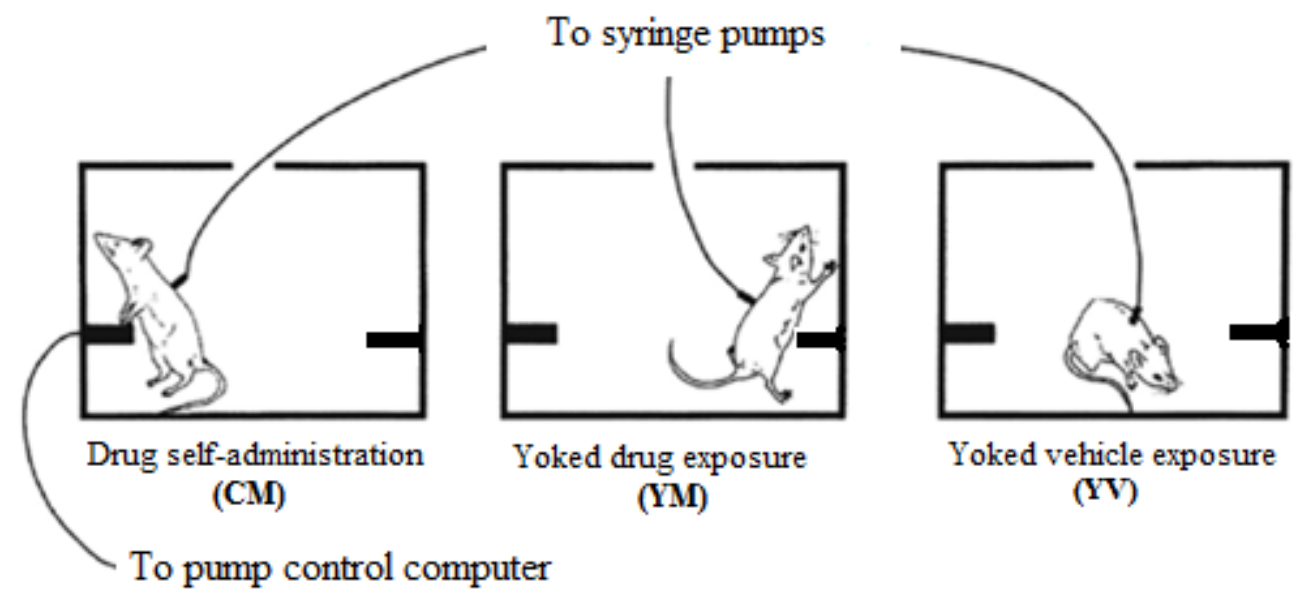

Figure 4. Triadic yoked MA self-administration. Contingent MA (CM; left) is delivered according to operant responding. Yoked MA (YM; centre) is delivered on the same frequency and pattern to the yoked rat dependent on responding of the rat receiving CM. Yoked vehicle (YV; right) infusions are delivered to the second yoked rat also dependent on CM delivery. A computer controlled syringe pump delivers all CM, YM and YV infusions. Image adapted from Haracz, Mash and Sircar (1999).

By using a yoked method of drug self-administration, it is possible to distinguish between two types of drug-induced neuroadaptations: the 
neurobiological consequences of drug exposure can be measured following YM exposure; compared to the effects involved in active drug self-administration behaviour, in addition to the pharmacological effects of the drug, measured following $\mathrm{CM}$ exposure; which can both be compared to any purely procedural effects following YV exposure.

The present study investigated the effects of MA self-administration on striatal DA and prefrontal cortex 5-HT levels. A yoked-control procedure was used to compare the effects of self-administered and passively administered MA. It was hypothesized that any neurochemical deficits following chronic MA selfadministration would be less pronounced than those seen following previous studies that examined effects of experimenter-administered MA, and may show more rapid recovery.

\section{Subjects}

Male Sprague-Dawley rats weighing approximately 200-230g $(n=40)$ were used in the acute experiment (experiment 1), whereas larger 300-350g $(n=63)$ rats were used for self-administration experiments (experiment 2). Smaller rats were used in experiment 1 due to the high mortality rate of larger/older rats exposed to this treatment protocol (Xi, et al., 2009). Additionally, rats of a similar age and size to those used in experiment 1 were not suitable for experiment 2 as our laboratory has found that they do not recover as well following catheter implantation (unpublished observations). Rats in experiment 1 were housed in groups of three prior to MA treatment. On the MA treatment day, each rat was housed in an individual cage due to the possibility of MA facilitating defensive behaviour and attacks (Maeda, Sato, \& Maki, 1985; Shintomi, 1975), and to minimize the effects of MA-produced hyperthermia (Askew, 1962). Individual housing conditions were maintained until brain tissue was harvested 24 hours, or seven days following treatment. Animals in experiment 2 were housed in groups of three to four prior to catheter implantation, and were then housed individually for the remainder of the experiment. 


\section{Experiment 1: MA self-administration}

\subsection{Yoked methamphetamine self-administration}

Yoked rats were run in groups of three during a 20 day period. One rat in each triad self-administered MA on an FR1 schedule of reinforcement (CM). Each active lever press by the CM triggered an infusion of MA (YM) or heparinised (3UI/ml) saline $0.9 \%$ (YV). Daily self-administration sessions were six hours long.

\section{Experiment 2: Experimenter-administered MA regimen}

Intraperitoneal (IP) injections of either the saline $0.9 \%$ vehicle $(n=20)$ or $4 \mathrm{x}$ $10 \mathrm{mg} / \mathrm{kg}$ MA were administered in the home cage every two hours $(n=20)$. Four animals died following the MA exposure protocol, however, leaving a total of $n=16$ for this group.

\section{Neurochemical procedures}

\subsection{Tissue extraction and preparation}

Rats were asphyxiated using $\mathrm{CO}_{2}$, decapitated, and their brains were rapidly removed either 24 hours, or seven days following the final MA self-administration session. Brains were then placed in a stainless steel block for slicing into $1 \mathrm{~mm}$ coronal sections (Heffner, Hartman, \& Seiden, 1980) that were placed onto an icechilled inverted petrie dish. The frontal cortex (FC; including prelimbic, cingulated and motor cortices) and striatum (Str; dorsal) were dissected out, immediately snapfrozen by immersion in liquid nitrogen and weighed. These regions were assayed as previous studies have suggested they are susceptible to MA-induced neurotoxicity (Friedman, et al., 1998; Graham, et al., 2008; Herring, et al., 2008). Tissue samples were then stored at $-80^{\circ} \mathrm{C}$ until further analyses were conducted. During storage, a Str sample from one control rat in the experimenter-administered group became compromised. Further, STR samples from one YV and one YM rat, and a FC sample from one YM rat in the self-administration groups became compromised. Data from these samples were excluded from analysis.

\subsection{Tissue analysis}

Tissue samples were homogenised in $0.1 \mathrm{~N}$ perchloric acid and centrifuged at $10000 \mathrm{~g}$ for 30 mins at $4^{\circ} \mathrm{C}$. The pellet was discarded, and the supernatant was filtered into vials. A $20 \mu \mathrm{l}$ aliquot was injected into a high-pressure liquid 
chromatography system with electrochemical detection (Agilent Eclipse EDB-C18, $150 \times 4.6 \mathrm{~mm}, 5 \mu \mathrm{m}$ granulometry). The mobile phase (75 mM sodium dihydrogen phosphate, $1.7 \mathrm{mM}$ octanesulphonic acid, $0.25 \mathrm{mM}$ Na2EDTA, $100 \mu \mathrm{L} / \mathrm{L}$ triethylamine, $10 \% \mathrm{v} / \mathrm{v}$ acetonitrile, $\mathrm{pH} 3.0$ ) was delivered at a constant flow rate of $1.0 \mathrm{ml} / \mathrm{min}$. Detection was performed using a coulometric detector (Coulochem III, ESA Inc., dual electrode detection cell, -175 and $+400 \mathrm{mV}$; guard cell electrode, +450 mV). Detection limits were $28 \mathrm{pg}$ for DA; $20 \mathrm{pg}$ for 5-HT; $12 \mathrm{pg}$ for HVA; and $10 \mathrm{pg}$ for 5-HIAA. Chromatograms were obtained using ChemStation software. Areas under the DA, 5-HT, HVA and 5-HIAA peaks for samples and for calibration standards were obtained. Concentrations of DA, 5-HT, HVA and 5-HIAA are expressed as ng/mg tissue, and were calculated according to the regression equations obtained with the calibration standards. Working external standards (500 - 15.125 $\mathrm{ng} / \mathrm{ml}$ in $0.1 \mathrm{~N}$ perchloric acid) were prepared fresh each day from external standard stock solutions.

\section{Statistical analyses}

The results from the experimenter-administered treatment regime were analyzed using 2 (condition: saline, MA) x 2 (withdrawal period: 24 hour, seven day) ANOVAs. Effects of withdrawal time were investigated using separate univariate ANOVAs.

The results from the self-administration experiment were analyzed using 3 (condition: CM, YM, YV) x 2 (withdrawal period: 24 hour, seven day) ANOVAs. Tukey's post-hoc analysis on effects of contingency was used when appropriate. Separate repeated measures ANOVAs (test day x lever) for each group (CM; YM; YV) were used to analyse differences in responding between active and inactive levers for the self-administration experiments. Separate univariate ANOVAs were used to determine significant differences between levers at each day. 


\section{Results}

\subsection{Methamphetamine self-administration}

\subsubsection{Yoked self-administration}

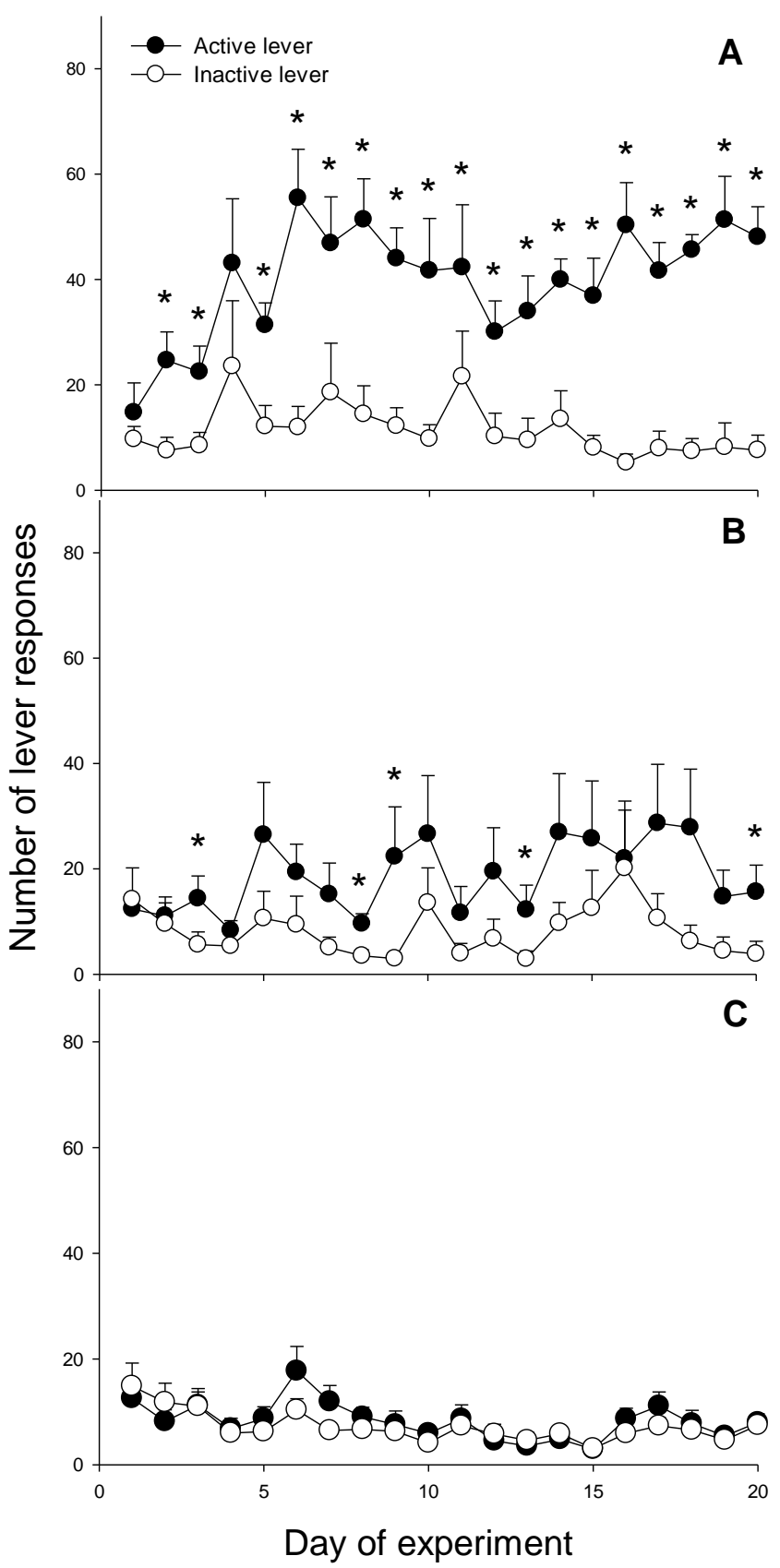

Figure 5. Average number of responses (+SEM) on inactive and active levers for $0.1 \mathrm{mg} / \mathrm{kg} / \mathrm{inf}$. MA during 6-hour sessions over a 20 day period. A) The contingent MA group (CM; $n=16)$. B) The yoked MA group $(\mathrm{YM} ; n=16)$. C) The yoked vehicle group ( $\mathrm{YV} ; n=16)$. *Significant difference between active and inactive levers $(p<0.05)$

Figure 5 (a: CM; b: YM; c: YC) shows the mean number of responses on the inactive and active levers during the 20 day self-administration period. The CM group rapidly acquired MA self-administration, as indicated by a preference for the 
active lever, and active lever responding increased over the first 6 days (fig. 7a). A repeated measures ANOVA showed a main effect of lever $[\mathrm{F}(1,30)=52.81, p<$ $0.0002]$, a main effect of day $[\mathrm{F}(19,570)=2.50, p=0.00004]$, and an interaction between these factors $[F(19,570)=1.96, p=0.007]$. Separate univariate ANOVAs revealed a significant difference between active and inactive levers from day 2 ( $p<$ 0.05), with the exception of day 4. The YM group also showed a significant preference for the active lever $[\mathrm{F}(1,30)=6.39, p=0.0351]$, but no effect of day $[\mathrm{F}(19,532)=0.92, p=0.563]$, and no interaction between these factors $[\mathrm{F}(19,570)$ $=0.72, p=0.529]$. Despite preferential responding on the active lever on some of the test days, the YM group did not fulfil criteria for MA self-administration acquisition because the preference for the active lever was erratic; separate univariate ANOVAs revealed that active lever preference was never maintained for three consecutive days (fig 7b). Finally, the YC group (fig. 7c) showed no preference for either lever $[\mathrm{F}(1,30)=0.44, p=0.782]$. There was a main effect of day $[\mathrm{F}(19,570)=5.50, p=0.00078]$, but no interaction between these factors $[\mathrm{F}(19$, $570)=1.15, p=0.41]$.

\subsection{Tissue concentrations of monoamines and metabolites}

\subsubsection{Self-administered/yoked MA exposure}

ANOVA revealed that tissue levels of DA in the dorsal Str were reduced as a function of MA exposure, confirmed by a main effect of treatment group $[\mathrm{F}(2,40)=$ $7.34, p=0.002]$. This reduction was transient and had returned to control levels by seven days, as demonstrated by a main effect of withdrawal period $[F(1,40)=10.84$, $p=0.002]$, but no interaction between these factors $[\mathrm{F}(2,40)=0.54, p=0.586]$. Tukey post-hoc tests showed no significant effect of contingency on striatal DA levels as $\mathrm{CM}$ and $\mathrm{YM}$ were not significantly different at either withdrawal period ( $p$ $=0.891)$. Because there was a main effect of withdrawal and treatment group, separate univariate ANOVAs were used to compare the CM and YM groups at both withdrawal times to the YV groups. Separate ANOVAs showed that DA levels in the $\mathrm{CM}[\mathrm{F}(1,14)=5.486, p=0.034]$, but not in the $\mathrm{YM}$ group $[\mathrm{F}(1,14)=3.324, p=$ 0.09] were significantly reduced at 24 hours. The reduction in the CM group recovered over the withdrawal period, however, as there was no longer a significant difference from the YV group at seven days $[\mathrm{F}(1,13)=4.162, p=0.062]$. 
Self-administered and yoked exposure to MA did not alter tissue levels of HVA in the $\operatorname{Str}[F(2,40)=2.14, p=0.131]$. On the other hand, DA turnover was significantly increased in MA-exposed groups $[\mathrm{F}(2,40)=11.51, p=0.000112]$, and there was a main effect of withdrawal $[\mathrm{F}(1,40)=6.94, p=0.012]$, and an interaction between these factors $[F(2,40)=4.99, p=0.012]$. Tukey's post hoc analysis showed no effect of contingency on DA turnover $(p=0.838)$. Because there was a main effect of withdrawal, separate ANOVAs were used to compare the CM and YM groups to YV groups. DA turnover was significantly elevated in both the $\mathrm{CM}[\mathrm{F}(1$, $14)=23.12, p=0.00028]$ and $\mathrm{YM}[\mathrm{F}(1,14)=20.86, p=0.00048]$ groups at 24 hours following the last self-administration session. This had returned to control levels at seven days, however, as neither $\mathrm{CM}[\mathrm{F}(1,13)=1.71, p=0.214]$, nor YM $[\mathrm{F}(1,13)=1.83, p=0.201]$ groups were significantly different from YV groups.

Tissue levels of 5-HT in the FC showed no effect of treatment group $[\mathrm{F}(2,41)$ $=2.26, p=0.118]$, or withdrawal time $[\mathrm{F}(1,41)=0.96, p=0.333]$. Equally, there were no significant effects of treatment group $[\mathrm{F}(2,41)=1.88, p=0.166]$, or withdrawal time $[\mathrm{F}(1,41)=0.67, p=0.417]$ on $\mathrm{FC}$ tissue levels of 5-HIAA, and no effect of treatment group $[\mathrm{F}(2,41)=2.14, p=0.131]$, or withdrawal time $[\mathrm{F}(1,41)=$ $0.14, p=0.713$ ] on 5-HT turnover. Table 2 shows the effect of self-administered MA on tissue levels of DA, 5-HT and their respective metabolites in dorsal Str and FC for the contingent MA (CM), yoked MA (YM) and yoked vehicle group (YV).

\subsubsection{Experimenter-administered MA}

ANOVA (condition $\mathrm{x}$ withdrawal time) revealed that DA levels in the dorsal Str were significantly reduced in MA-exposed groups $[\mathrm{F}(1,31)=15.50, p=$ 0.00045]. Effects at the 24 hour and seven day withdrawal periods were comparable and there was no significant effect of this variable $[\mathrm{F}(1,31)=2.80, p=0.104]$, or an interaction between these factors $[\mathrm{F}(1,31)=0.25, p=0.619]$. ANOVA on tissue levels of homovanillic acid (HVA) failed to reveal an effect of condition $[\mathrm{F}(1,31)=$ $0.05, p=0.822]$, withdrawal time $[\mathrm{F}(1,31)=0.20, p=0.889]$, or an interaction between these factors $[\mathrm{F}(1,31)=0.62, p=0.436]$. DA turnover (HVA/DA) was, however, significantly increased in MA pre-treated groups, and there was a main effect of treatment group $[\mathrm{F}(1,31)=21.65, p=0.00058]$, withdrawal time $[\mathrm{F}(1,31)$ $=9.65, p<0.004]$, and an interaction between these factors $[\mathrm{F}(1,31)=7.90, p<$ 0.008]. Because there was a main effect of withdrawal, separate univariate ANOVAs 
were used to compare the MA pre-treated groups at both withdrawal times to the saline pre-treated groups. ANOVA revealed that DA turnover was significantly elevated at the 24 hours $[\mathrm{F}(1,16)=19.215, p=0.00046]$, but not the seven day withdrawal period $[\mathrm{F}(1,15)=3.431, p=0.084]$.

Analysis of tissue levels of 5-HT in the FC revealed a significant depletion among MA exposed groups $[\mathrm{F}(1,32)=5.47, p=0.026]$, that was persistent as there was no effect of withdrawal period $[\mathrm{F}(1,32)=1.36, p=0.252]$, and no interaction between these factors $[\mathrm{F}(1,32)=1.24, p=0.274]$. There were no effects on 5hydroxyindoleacetic acid (5-HIAA) as a function of treatment group $[\mathrm{F}(1,32)=3.14$, $p=0.086]$, or withdrawal time $[\mathrm{F}(1,32)=2.20, p=0.148]$, and no interaction between these factors $[\mathrm{F}(1,32)=2.94, p=0.096]$. There were also no effects on 5HT turnover (5-HIAA/5-HT) as a function of MA exposure $[\mathrm{F}(1,32)=0.18, p=$ $0.675]$, or withdrawal time $[\mathrm{F}(1,32)=0.02, p=0.885]$, nor an interaction between these factors $[\mathrm{F}(1,32)=4.09, p=0.051]$. Table 3 shows the effect of the high-dose treatment of MA on tissue levels of DA, 5-HT and their respective metabolites in dorsal Str, and FC, respectively, following either a 24 hour (MA24hr) or seven day (MA7day) drug-free period. 


\begin{tabular}{|c|c|c|c|c|c|c|c|}
\hline \multirow[t]{2}{*}{ Region } & & \multicolumn{2}{|c|}{ DA } & \multicolumn{3}{|c|}{ HVA } & \multirow{2}{*}{$\begin{array}{l}\text { HVA/DA } \\
7 \text { days }\end{array}$} \\
\hline & & 24 hours & 7 days & 24 hours & 7 days & 24 hours & \\
\hline \multirow[t]{5}{*}{ Striatum } & $\mathbf{Y V}$ & $15.55 \pm 0.92$ & $17.75 \pm 0.97$ & $1.01 \pm 0.09$ & $1.23 \pm 0.08$ & $0.06 \pm 0.00$ & $0.07 \pm 0.00$ \\
\hline & $\mathbf{C M}$ & $12.56 \pm 0.89 *$ & $15.32 \pm 0.72$ & $1.27 \pm 0.11$ & $1.22 \pm 0.07$ & $0.10 \pm 0.01 \ddagger$ & $0.08 \pm 0.00$ \\
\hline & $\mathbf{Y M}$ & $13.74 \pm 0.39$ & $14.93 \pm 0.47$ & $1.43 \pm 0.11$ & $1.19 \pm 0.10$ & $0.10 \pm 0.01 \ddagger$ & $0.08 \pm 0.01$ \\
\hline & & \multicolumn{2}{|c|}{ 5-HT } & \multicolumn{3}{|c|}{ 5-HIAA } & 5-HIAA/5-HT \\
\hline & & 24 hours & 7 days & 24 hours & 7 days & 24 hours & 7days \\
\hline \multirow[t]{3}{*}{ Frontal Cortex } & $\mathbf{Y V}$ & $0.47 \pm 0.03$ & $0.52 \pm 0.04$ & $0.48 \pm 0.04$ & $0.46 \pm 0.02$ & $1.04 \pm 0.09$ & $0.91 \pm 0.08$ \\
\hline & $\mathbf{C M}$ & $0.41 \pm 0.05$ & $0.44 \pm 0.01$ & $0.42 \pm 0.03$ & $0.42 \pm 0.03$ & $1.12 \pm 0.12$ & $1.03 \pm 0.06$ \\
\hline & $\mathbf{Y M}$ & $0.49 \pm 0.03$ & $0.49 \pm 0.04$ & $0.39 \pm 0.02$ & $0.44 \pm 0.03$ & $0.82 \pm 0.04$ & $0.95 \pm 0.12$ \\
\hline
\end{tabular}

Table 2. Average $( \pm$ SEM) monoamine and metabolite levels (ng/mg tissue) for the yoked vehicle (YV) groups contingent MA (CM) groups, and yoked MA (YM) groups at 24 hours and seven days following final self-administration session. Symbols show a significant difference relative to YV group: $*(p<0.05) ; \ddagger(p<$ 0.001). 


\begin{tabular}{|c|c|c|c|c|c|c|c|}
\hline & & & DA & & HVA & & HVA/DA \\
\hline & & 24 hours & 7 days & 24 hours & 7 days & 24 hours & 7 days \\
\hline \multirow[t]{4}{*}{ Striatum } & Saline & $11.85 \pm 0.57$ & $13.05 \pm 0.65$ & $1.14 \pm 0.06$ & $1.22 \pm 0.07$ & $0.10 \pm 0.01$ & $0.09 \pm 0.00$ \\
\hline & MA & $7.27 \pm 1.83 \dagger$ & $9.51 \pm 0.83 \dagger$ & $1.21 \pm 0.23$ & $1.09 \pm 0.08$ & $0.22 \pm 0.03 \ddagger$ & $0.12 \pm 0.02$ \\
\hline & & & 5-HT & & 5-HIAA & & 5-HIAA/5-HT \\
\hline & & 24 hours & 7 days & 24 hours & 7 days & 24 hours & 7days \\
\hline \multirow[t]{2}{*}{ Frontal Cortex } & Saline & $0.39 \pm 0.01$ & $0.40 \pm 0.01$ & $0.37 \pm 0.02$ & $0.44 \pm 0.02$ & $0.98 \pm 0.08$ & $1.14 \pm 0.06$ \\
\hline & MA & $0.33 \pm 0.03 *$ & $0.37 \pm 0.02 *$ & $0.37 \pm 0.02$ & $0.37 \pm 0.03$ & $1.19 \pm 0.10$ & $1.00 \pm 0.10$ \\
\hline
\end{tabular}

Table 3. Average ( \pm SEM) monoamine and metabolite levels (ng/mg tissue) for the saline and methamphetamine (MA) pre-treated groups. Symbols show a significant difference relative to saline groups: * $(\mathrm{p}<0.05)$; $\uparrow(\mathrm{p}<0.01)$; $*(\mathrm{p}<0.001)$. 


\section{Discussion}

\subsection{Behavioural results}

\subsubsection{Yoked self-administration tests}

MA self-administration was rapidly acquired by the contingent MA group $(\mathrm{CM})$, as demonstrated by preferential responding on the active lever from day 2 , and throughout the 20 day experiment, apart from day 4. The yoked MA group (YM) also showed a significant preference for the active lever. However, the preference was sporadic and never met the criteria for acquisition of selfadministration behaviour. CM and YM groups were exposed to a daily average of 4$5 \mathrm{mg} / \mathrm{kg}$ MA. Our laboratory has previously demonstrated increased stereotypy and locomotor activity at doses of $2 \mathrm{mg} / \mathrm{kg}$ MA (Brennan, Johnstone, Fitzmaurice, Lea, \& Schenk, 2007), suggesting the increased lever pressing in the YM group may reflect elevated general behaviour activation as a result of MA intoxication. However, this does not explain the preference for the active lever. Rather, this may be clarified by an association between the stimulus light paired with each drug infusion and the subjective drug effects. During the yoked experiment, whenever the $\mathrm{CM}$ responded on the active lever to deliver an infusion of MA, the stimulus light above this lever was illuminated in each chamber of the triad. This pairing between light illumination and drug infusion may have produced a conditioned drugassociation for the stimulus light, engendering approach behaviours in the YM toward the stimulus light and active lever (right corner of test chamber). Stereotyped head-bobbing (as observed upon video recording of the test chambers), produced by MA intoxication, in the right corner of the test chamber would produce the erratic, rather than focused, active lever presses. The MA-produced behavioural activation in concert with a conditioned preference for the right corner of the test chamber may explain the sporadic preference for the active lever.

\subsection{Neurochemical results}

\subsubsection{Chronic self-administration exposure}

Self-administered MA produced transient decreases in striatal DA in addition to a transient increase in DA turnover. These findings are consistent with results from another study on the neurochemical effects of self-administered MA. 
Decreased striatal and prefrontal cortex DAT densities, but no changes in striatal DA or prefrontal cortex 5-HT levels, or any changes in typical markers of neurotoxicity (e.g. tyrosine hydroxylase levels, loss of terminal fibres, silver staining, glial fibrillary acidic protein, or calcium-binding adaptor molecule 1 levels) were reported following a 14-day withdrawal period (Schwendt, et al., 2009). Longer session durations have produced more extensive reductions in monoamine tissue levels, as 15 hours of access to self-administered MA for eight days, produced persistent reductions in DA and DAT levels up to 14 days following the final session (Krasnova, et al., 2010). Nonetheless, changes in monoamine levels following MA self-administration do not necessarily equate to neurotoxic damage and appear less pronounced compared to the persistent reductions in DA levels following experimenter-administered MA regimes at three (Chapman, et al., 2001) and up to seven weeks (Friedman, et al., 1998) following MA exposure. Together, these findings suggest that MA self-administration under the current conditions did not produce extensive neurotoxicity and/or any lasting neurochemical disruption. However, the transient DA reductions and increase in DA turnover found in the present study, and the lasting decreases in DAT densities (Schwendt, et al., 2009), and more persistent reductions in DA and DAT levels following a longer session duration (Krasnova, et al., 2010) suggest that both short-term and enduring alterations in the regulation of DA neurotransmission occur as a consequence of selfadministered MA exposure.

These changes in DA regulation may have functional implications. Indeed, exposure to psychostimulants at doses that only produce transient monoamine reductions, in the absence of any markers of neurotoxic damage, may have persistent effects on behaviour and cognition (Baumann, Wang, \& Rothman, 2007; Dalley, et al., 2007). For instance, six hours access to MA self-administration produced an impairment in a novel object recognition task 10 days after the final session, enhanced MA-primed reinstatement of drug-seeking (Rogers, et al., 2008), and an increase in MA self-administration compared to one hour access (Kitamura, et al., 2006). Furthermore, eight hours access to self-administered MA produced significant impairment (i.e. accuracy reduction, omissions, disturbed response latency and increased impulsivity) on a five-choice serial reaction time task up to two weeks following the final self-administration session (Dalley, et al., 2007). This would suggest that prolonged access to contingent MA may increase cognitive 
deficits, impulsivity, and motivation for MA-seeking, impair attention and perpetuate an increase in MA self-administration. Indeed, MA users who had experienced a discrete period of unrestricted access to MA in the past reported greater motivation for continued MA use (more days of use per week, greater use per day, and greater total use per week), compared to MA users with no experience of unrestricted access (Culbertson, De La Garza II, Costello, \& Newton, 2009).

Conversely, self-administered MA failed to alter tissue levels of 5-HT, 5HIAA, or rate of 5-HT turnover in the FC. These results are not entirely unexpected since the experimenter-administered treatment protocol produced more substantial depletions of striatal DA tissue levels compared to cortical 5-HT levels. Given that striatal dopaminergic systems have been suggested to be the most sensitive to MAproduced neurochemical perturbations (Chu, et al., 2008; J. E. Hanson, et al., 2009; Quinton \& Yamamoto, 2006; Thomas, et al., 2008; Wagner, et al., 1980) it was expected that greater effects would be seen here as opposed to cortical regions. Further, a reduced density of DATs in the striatum and prefrontal cortex following self-administered MA occurred in the absence of any changes to SERT in these regions (Schwendt, et al., 2009). These findings are consistent with the idea that MA self-administration produces predominantly dopaminergic effects.

\subsubsection{Yoked self-administration exposure}

A yoked self-administration protocol was used to investigate the effect of contingency on brain chemistry. There was no difference between contingent (CM group) and yoked delivery (YM group) of MA on tissue levels of monoamines and metabolites, or turnover rates. The present lack of significant difference between exposure protocols may relate to the mode of action of MA and the present neurochemical assay. Studies of experimenter-administered MA have shown that MA functions as a DA uptake blocker, a potent releaser of DA, decreases striatal DAT activity and reduces DA tissue levels (Bakit, et al., 1974; Cruickshank \& Dyer, 2009; Fleckenstein, et al., 2000; Julien, 2001; Krasnova \& Cadet, 2009; Suzuki, et al., 1980). More specifically, MA enters the presynaptic terminal button via the DAT, and binds with the VMAT-2, reversing its action and expelling DA in the cytosol, where it is pumped into the synaptic cleft via MA-induced reverse transport through the DAT (Barr, et al., 2006; Cruickshank \& Dyer, 2009; Kish, 2008). Moreover, MA also limits monoamine metabolism by inhibiting MAO, further increasing the pool 
of DA available for reverse transport into the synapse and duration for receptor activation (Cruickshank \& Dyer, 2009; Elkashef, 2008; Julien, 2001; Suzuki, et al., 1980). These combined effects contribute to a drastic increase in synaptic monoamine levels, and the longer elimination half-life (11-12 hrs in humans) than most other psychostimulants, such as cocaine (1-2 hours; Newton, et al., 2005). The present transient reductions in DA tissue levels and increased rate of DA turnover seen in rats exposed to both contingent and yoked MA infusions, may, therefore, reflect the purely pharmacological effects of chronic MA exposure per se, rather than the differences between the motivated process of active MA-taking behaviour and passive MA exposure. Other studies have also shown neuroadaptational differences in rats exposed to contingent and yoked drug infusions, compared to yoked controls. DA D1-like receptor densities, for instance, were reduced in rats receiving response contingent as well as yoked infusions of cocaine (De Montis, Co, Dworkin, \& Smith, 1998). Furthermore, the maximum number of D1 receptor binding sites $\left(B_{\max }\right)$, and DA stimulated adenylyl cyclase activity $\left(V_{\max }\right)$ was similarly reduced in rats exposed to both contingent and yoked cocaine infusions (De Montis, et al., 1998). That these neuroadaptations occurred in rats receiving both contingent and yoked cocaine infusions suggests they reflect the purely pharmacological effects of self-administered cocaine exposure, rather than cognitive processes involved in drug-taking.

Given the relatively non-specific nature of monoamine tissue content analysis using HPLC with neurochemical detection, the lack of significant differences between contingent and yoked MA exposure is not unexpected. For instance, this neurochemical assay does differentiate between intracellular and extracellular DA levels, and the purely pharmacological effects of MA may render differences in exposure contingency on the present measures undetectable. Indeed, many studies showing differences between contingency of drug exposure have examined different measures, such as changes in receptor density, receptor binding, and gene expression, or used in vivo microdialysis to detect changes in monoamine levels during drug self-administration. For instance, in vivo microdialysis revealed greater increases in DA levels in rats actively self-administering cocaine, compared to yoked littermates (Hemby, et al., 1997). Quantitative autoradiography showed that only contingent access to self-administered MA reduced DA D1- and D2-like receptor densities (Stefanski, et al., 1999), whereas only yoked cocaine infusions 
produced a reduction in D2-like receptor densities (Stefanski, et al., 2007). Furthermore, in situ hybridization analysis revealed that only contingent cocaine increased D2 receptor mRNA levels, with no corresponding changes in D2-like receptor binding density, compared to yoked littermates (Stefanski, et al., 2007). These findings suggest that behaviourally contingent drug exposure produces different neurobiological consequences, as measured by changes in receptor disposition and gene expression, to those seen following passive exposure, and emphasize the importance of using appropriate behavioural models when investigating the consequences of MA dependence.

There is a relative paucity of studies using self-administered MA to investigate neurobiological consequences of this exposure, whereas ED protocols are more prolific. ED protocols have been used to provide a greater approximation of the escalation in dose and pattern of MA exposure seen in MA dependence, than typical single-day acute high dose exposure, but still require non-contingent MA administration. Indeed, rats that underwent ED regimes were exposed to comparable and greater amounts of total MA than in the present study (Cadet, et al., 2009; Danaceau, et al., 2007; Fischman \& Schuster, 1974; Graham, et al., 2008; O'Neil, et al., 2006; Segal, et al., 2003), yet displayed no changes in striatal DA levels or DA turnover at 24 hours following the final treatment (Danaceau, et al., 2007; Graham, et al., 2008; O'Neil, et al., 2006). The divergence between passively-administered and actively self-administered neurochemical consequences of MA exposure may best be explained by the relatively low starting doses of MA that are typically employed in ED regimes. It has previously been suggested that the typical low doses of an ED regimen may allow a tolerance to the DA-depleting effects of MA to develop (Cadet, et al., 2009; Danaceau, et al., 2007; Fischman \& Schuster, 1974; O'Neil, et al., 2006; Segal, et al., 2003). On the other hand, the rats selfadministering MA in the present study were exposed to comparatively high initial doses of MA, due to the rapid acquisition of MA self-administration and escalation in responding over the first six days (fig. 5a). Indeed, self-administration session duration has determined the amount of daily MA intake, where two hour sessions resulted in circa 1-2 mg/kg (Stefanski, et al., 1999), six hour sessions 4-5 mg/kg (present findings), nine hour sessions $6 \mathrm{mg} / \mathrm{kg}$ (Shepard, et al., 2006), and 15 hour sessions approximately $14-15 \mathrm{mg} / \mathrm{kg}$ (Krasnova, et al., 2010) of daily MA intake. In addition, longer session duration facilitates acquisition of MA self-administration 
(Kitamura, et al., 2006; Rogers, et al., 2008), and the rapid acquisition and escalation of responding in the present study permitted a higher initial MA dose exposure that may account for the transient striatal DA deficits.

\subsubsection{Experimenter-administered MA exposure}

The persistent reductions of DA and 5-HT that occurred following the MA 'binge' treatment regime are consistent with the findings of other studies (Chapman, et al., 2001; Friedman, et al., 1998; Fuller \& Hemrick-Leuke, 1980; J. E. Hanson, et al., 2009; Herring, et al., 2008; Hotchkiss \& Gibb, 1980; Hotchkiss, et al., 1979; Quinton \& Yamamoto, 2006). Because MA-produced hyperthermia exacerbates neurotoxicity and DA depletions, and ambient temperature affects the degree of MA-produced hyperthermia (Imam \& Ali, 2001; Krasnova \& Cadet, 2009), the experimenter-administered treatment regime was administered in a temperaturecontrolled housing room. Nonetheless, the high MA dose (4 x $10 \mathrm{mg} / \mathrm{kg})$ was lethal to $20 \%$ of the rats.

DA turnover (HVA/DA) was transiently elevated in MA-exposed animals, whereas other studies have shown more persistent elevations in DA turnover (Cadet, et al., 2009; Davidson, et al., 2005; Friedman, et al., 1998). The more transient effect seen here may be explained by the use of relatively young rats in this group, as MAproduced DA deficits and changes in DA regulation are more pronounced in older rats. Indeed, high dose MA injections (40 mg/kg x 1; $10 \mathrm{mg} / \mathrm{kg}$ x 4 every $2 \mathrm{hrs}$ ) caused death in the majority of adult rats at similar weights to those used in the present self-administration study (275-300 g; Xi, et al., 2009). A single high dose of MA (40 mg/kg, IP) caused $100 \%$ mortality in 12-month-old rats, whereas no deaths occurred in one or six month old rats (Imam \& Ali, 2001). Furthermore, there was an age-related increase in the reduction of DA and its metabolites in the Str following $40 \mathrm{mg} / \mathrm{kg}$ MA (IP), and following $5 \mathrm{mg} / \mathrm{kg}$ MA (IP) there was no effect observed in one month old rats, whereas the effect was significant in rats aged six and 12 months (Imam \& Ali, 2001). These age-related differences may be attributed to more efficient DA neurotransmission in younger rats (Thomas, et al., 2008; Volz, Farnsworth, Rowley, Hanson, \& Fleckenstein, 2009). More specifically, younger (postnatal day 38-42) compared to older (postnatal day 88-92) rats appear to have kinetically up-regulated VMAT-2 and higher levels of functionally active DAT (Volz, et al., 2009). In the presence of MA, this may enable greater cellular efflux of 
DA from the cytoplasm, thereby preventing cytoplasmic DA rising to neurotoxic levels after drug administration.

Because tissue levels of HVA were not affected by MA treatment in the present study, increased DA turnover could be interpreted as a simple depletion of DA in the absence of any change in HVA levels. As DA levels in the striatum were greatly reduced, however, and because HVA is a catabolite of DA, it is possible that the rate of DA breakdown was increased as a consequence of MA exposure. Indeed, the enzymes responsible for breaking DA down to HVA were up-regulated under conditions of increased microglial activity (Helkamaa, et al., 2007; Thomas, et al., 2008), such as that following MA exposure (Herring, et al., 2008; Krasnova \& Cadet, 2009; Krasnova, et al., 2010). The increased DA turnover could augment the production of free radical intermediates, such as the potentially toxic compounds hydrogen peroxide, ammonia, and aldehyde (Holshneider \& Shih, 2000), thereby increasing the risk of further oxidative injury (Hauptmann, Grimsby, Shih, \& Cadenas, 1996; Wei, Yeung, Jurma, \& Andersen, 1996). This suggests that, in the short-term, the MA-induced increase in DA turnover seen here could potentiate striatal neuronal damage.

The experimenter-administered treatment regime also produced a persistent depletion of 5-HT levels in the frontal cortex. A report of rapid recovery of significant 5-HT depletions (>80\%) two hours following high-dose MA treatment (Graham, et al., 2008), suggests that neurochemical assay at an earlier time point may have revealed even more substantial deficits than those seen here. In addition, 5-HIAA levels and 5-HT turnover were not affected in the present study, suggesting that experimenter-administered MA did not produce lasting changes in frontal cortex serotonergic neurotransmission. Taken together, these results suggest that dorsal striatal dopaminergic systems are more susceptible to neuronal damage produced by MA-exposure, than frontal cortical serotonergic systems (Chu, et al., 2008; J. E. Hanson, et al., 2009; Quinton \& Yamamoto, 2006; Thomas, et al., 2008; Wagner, et al., 1980).

In conclusion, the present study makes a significant contribution to drug dependence research by presenting an extended-access yoked-animal model that is more representative of human MA dependence. The findings presented here revealed that the striatal dopaminergic deficits produced by MA self-administration were comparatively mild, because they were no longer evident at seven days. This is 
consistent with previous studies suggesting that exposure to self-administered MA does not produce the extensive reductions in monoamine tissue levels and neurotoxicity observed following experimenter-administered high-dose MA treatment regimes. Specifically, self-administered MA produced a transient reduction of striatal DA tissue levels, and increase in DA turnover that may suggest a disruption of DA neurotransmission and regulation in the dorsal striatum that recovers over time. There was no effect of contingency on these measures as comparison between contingent and yoked self-administration groups revealed no significant differences. This suggests that divergent neuroadaptations following dissimilar chronic MA regimens may be for the most part due to varying levels and time course of MA exposure.

\section{Chapter 2: Effects of D1-like and D2-like receptor antagonists on MA self-administration}

\section{Overview}

MA self-administration during six hour sessions reduced DAT densities, that persisted up to 14 days (Schwendt, et al., 2009), increased DA turnover rates, and produced transient reductions in tissue levels of DA (Chapter 1). MA selfadministration during two hour sessions, also produced a reduction in D1- and D2like receptor binding densities 24 hours, but not seven days, following the last selfadministration session (Stefanski, et al., 1999; Stefanski, et al., 2002). These transient neuroadaptations may have functional implications for MA dependence because D1- and D2-like receptor mechanisms have been suggested to mediate crucial aspects of drug self-administration (Martinez, et al., in press; Missale, et al., 1998; Self, 1998). Pharmacological manipulations targeting D1- and D2-like receptor subtypes provide a means of investigating the role of these receptor mechanisms in MA self-administration.

Most studies on the effect of DA receptor antagonists in the maintenance of psychostimulant self-administration and their reinforcing effects have studied cocaine self-administration, and both D1- and D2-like receptor subtypes have been implicated (Barrett, et al., 2004; Hubner \& Moreton, 1991; McGregor \& Roberts, 1995; Self, 2004b). For instance, when cocaine self-administration was maintained 
by simple FR schedules of reinforcement, both D1- and D2-like receptor antagonists shifted the dose-response curve downward and to the right (Caine \& Koob, 1994; Caine, et al., 1999). Further, the reinforcing efficacy of, and incentive motivation for cocaine, as measured by BPs on a PR schedule of reinforcement, was reduced by D1- (McGregor \& Roberts, 1995; Ranaldi \& Wise, 2001) and D2-like receptor antagonists (Bari \& Pierce, 2005; Hubner \& Moreton, 1991). A small number of studies suggest a role of DA in MA self-administration as responding on both FR and PR schedules of reinforcement has been altered by pre-treatment with DA antagonists (Wee, et al., 2007; Yokel \& Wise, 1976). The selective contribution of D1- vs. D2-like receptor mechanisms to self-administration of MA, however, is not understood.

The aim of Chapter 2 was, therefore, to investigate the contribution of DA D1- and D2-like receptors to MA self-administration. This was achieved in the third experiment by analyzing changes in the number of responses maintained by an FR1 schedule of reinforcement following pre-treatment with a D1- or D2-like receptor antagonist. The fourth experiment generated a dose-response curve of BPs under a PR schedule of reinforcement. The MA dose that generated the highest BP was then used for further investigation of the contribution of D1- and D2-like receptors to MA's reinforcing efficacy. SCH 23390 and eticlopride are highly selective antagonists at DA D1- and D2-like receptors, respectively (Bourne, 2001; Martelle \& Nader, 2008), and were used to investigate the contribution of these receptor subtypes to the maintenance of MA self-administration under both the FR and PR schedules of reinforcement. Because two hour MA self-administration sessions produced down-regulated D1- and D2-like receptor densities, but no effects on DAT and TH levels (Stefanski, et al., 1999), the present experiments used a two hour session duration in order to minimize dopaminergic deficits and neurotoxicity due to MA self-administration, and elucidate the functional implications of DA receptor down-regulation. Two hour MA self-administration sessions produced greater reductions in D2- over D1-like receptor binding densities (Stefanski, et al., 1999), suggesting D2-like receptor mechanisms may be less responsive to pharmacological manipulations. It was, therefore, hypothesized that greater effects on MA selfadministration would be observed following D1-like antagonist pre-treatment. 


\section{Subjects}

Adult male Sprague-Dawley rats were used for self-administration experiments in accordance with the general methods ( $n=22$ for FR experiments; $n=$ 18 for PR experiments).

\section{Experiment 3: Effects of D1- and D2-like Receptor Antagonists on Methamphetamine Self-administration on an FR1 Schedule of reinforcement}

Following acquisition, tests were conducted to assess the effects of $\mathrm{SCH}$ 23390 and eticlopride on responding maintained by a range of MA (0.05-0.2 $\mathrm{mg} / \mathrm{kg} /$ infusion) doses. SCH $23390(0.01-0.02 \mathrm{mg} / \mathrm{kg}, \mathrm{SC}, n=10)$ was administered in the home cage $15 \mathrm{~min}$ prior to the onset of the self-administration test session. These doses and pre-treatment times were based on previous studies from our laboratory on the effects of SCH 23390 on MDMA (Daniela, et al., 2004), and cocaine (Brennan, Lake, et al., 2007) self-administration. Tests of the effect of eticlopride $(0.0-0.05 \mathrm{mg} / \mathrm{kg}, \mathrm{IP}, n=12)$ on responding maintained by MA were conducted in a separate group of rats. Procedures were identical to those used for SCH 23390 tests, except that eticlopride was administered in the home cage $30 \mathrm{~min}$ prior to the onset of self-administration testing. These doses and pre-treatment times were based on previous studies from our laboratory on MDMA self-administration (Brennan, et al., 2009) and reinstatement (Schenk \& Gittings, 2003).

A recurring series of tests comprised of baseline and test days ensued. Antagonists were only administered when there were at least two prior consecutive baseline test days during which responding on the active lever did not vary by more than $20 \%$.

The initial test dose of MA was $0.05 \mathrm{mg} / \mathrm{kg} / \mathrm{infusion}$. Once the effect of all doses of the antagonist, administered in random order, on responding maintained by this dose had been determined, the dose was increased to 0.1 or $0.2 \mathrm{mg} / \mathrm{kg} /$ infusion for individual rats, and testing of the antagonist on responding maintained by this dose commenced. Only 


\section{Experiment 4: Effects of D1- and D2-like Receptor Antagonists on Methamphetamine Self-administration on a PR Schedule of reinforcement}

\subsection{Training}

Following acquisition of self-administration, an FR2 schedule of reinforcement was imposed until there was less than $20 \%$ variation across three days. The reinforcement requirements were then increased to FR5 for a minimum of five days. Once responding was stable ( $<20 \%$ variation across the last three days) the schedule of reinforcement was changed to PR, and the $30 \mathrm{sec}$ TO period was omitted. One experimenter triggered infusion was delivered to clear the catheter line of the heparinised solution at the start of each daily session. The next infusion was delivered contingent on a single active lever response. Thereafter, the number of responses required to obtain subsequent infusions was increased according to the following progression: 2, 4, 6, 9, 12, 15, 20, 25, 32, 40, 50, 62, 77, 95, 118, 145, 178, 219, 268, 328, 402, 492, 603, 737, 901, 1102, 1347, 1647, 2012. This PR series was derived from the following equation: Response ratio (rounded to nearest integer $)=\left[5 e^{(\text {injection number x 0.2) }}\right]-5$

This series was used because it has been used extensively in studies of cocaine and MA self-administration (Brebner, Phelan, \& Roberts, 2000; Richardson \& Roberts, 1996; Roberts, et al., 1996).

The ratio continued to increase within session until it failed to be completed within a one hour period. If any individual session continued for more than six hours, a water spout was inserted into the experimental chamber for the remainder of the session. If any individual session continued for longer than 12 hours, testing was omitted the following day. BP was defined as the last ratio completed during a session. Inactive lever responding for the PR experiments was not recorded.

\subsection{Effects of D1- and D2-like antagonism}

The dose response curve for MA self-administration was generated across days. The first dose tested was $0.1 \mathrm{mg} / \mathrm{kg} / \mathrm{infusion}$. Tests of this dose continued for at least five days, or until there were three consecutive days during which ranked BP did not vary by more than $20 \%$. The dose of MA for each individual rat was then changed to 0.05 , or $0.2 \mathrm{mg} / \mathrm{kg} /$ infusion. This procedure was repeated until all rats had been tested on each dose of MA $(n=12)$. Some rats failed to meet the 
responding stability criteria and were excluded from the study. Tests of the effects of SCH 23390 (0.0-0.04 mg/kg, SC, $n=6)$, and eticlopride (0.0-0.05 mg/kg, IP, $n=6)$ on self-administration of the dose of MA that generated the highest BPs $(0.2$ $\mathrm{mg} / \mathrm{kg} /$ infusion) were then conducted.

For these tests, a recurring series comprised of baseline and test days ensued. A minimum of two baseline testing days, in which there was less than $20 \%$ variation in number of infusions obtained, were given between tests of antagonist effects. SCH 23390 and eticlopride administration was identical to that in the FR experiments. Doses were administered in random order.

\section{Drugs}

SCH 23390 and eticlopride (Sigma Aldrich, Australia) were dissolved in a sterile $0.9 \%$ saline solution, and injected in a volume of $1 \mathrm{ml} / \mathrm{kg}$. All drug doses refer to the weight of the salt.

\section{Statistical analyses}

Effects of the D1- and D2-like receptor antagonists on MA selfadministration reinforced according to the FR1 schedule were analysed using separate ANOVAs (antagonist dose x MA dose) to determine the effect of each antagonist, with Tukey's post-hoc analyses conducted where appropriate. Further, two-way ANOVAs (antagonist dose x lever) were used to determine the effects of each antagonist on active vs. inactive lever responding. The dose response curve for MA self-administration reinforced according to the PR schedule was analyzed using a repeated measures ANOVA, and $t$-tests to determine differences between doses. Separate repeated measures ANOVAs, with post hoc $t$-tests where appropriate, were used to determine the effect of each antagonist on BPs maintained by MA on the PR schedule of reinforcement. 


\section{Results}

\subsection{Responding on the FR1 schedule of reinforcement}

\subsubsection{Effects of SCH 23390}

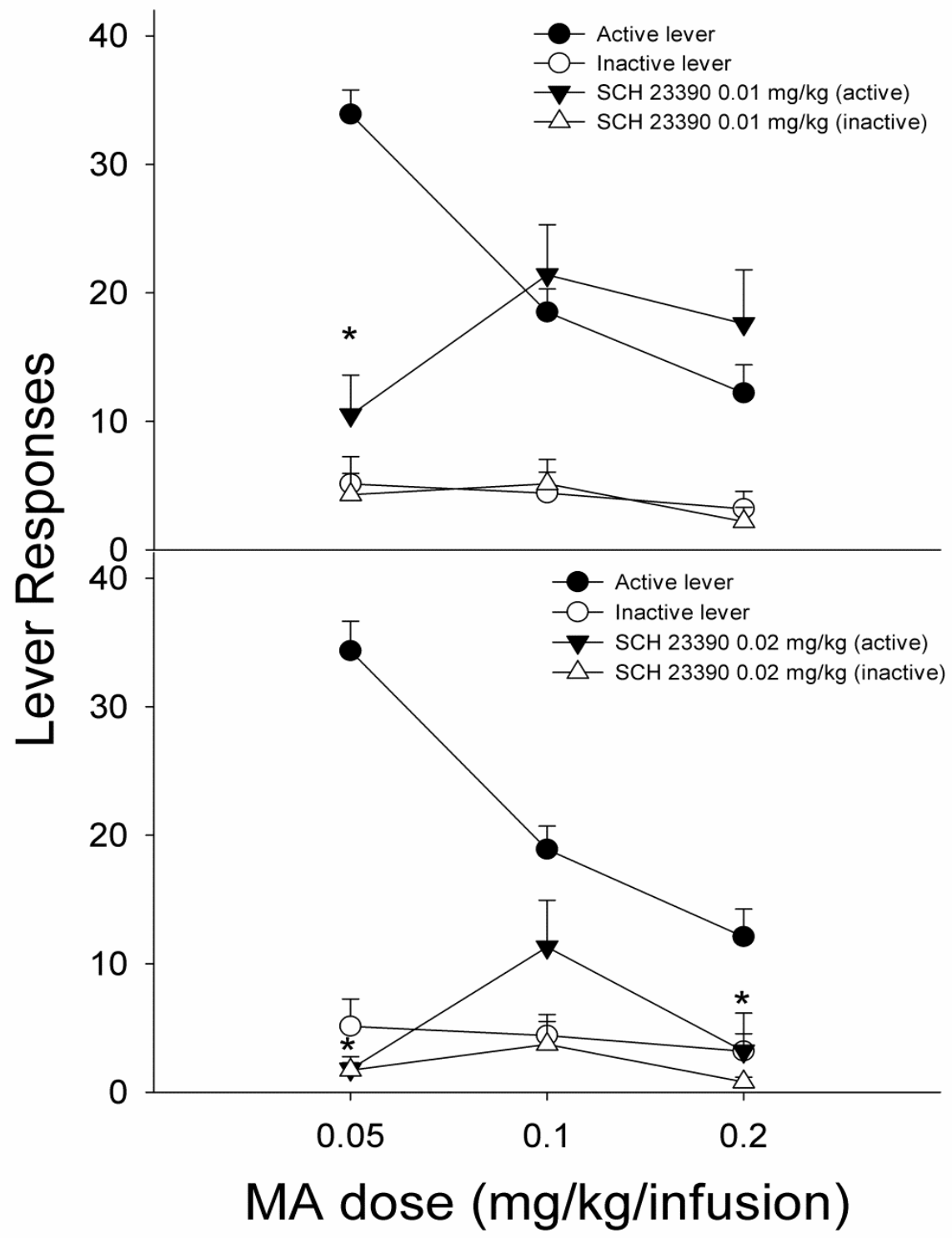

Figure 6. Effects of two doses of SCH 23390 [A) 0.01 ; B) $0.02 \mathrm{mg} / \mathrm{kg}$ ] on lever responding maintained by various doses of MA $(0.05,0.1,0.2 \mathrm{mg} / \mathrm{kg} /$ infusion $)$. Symbols represent mean number of responses (+SEM). *Significant difference between vehicle and SCH 23390 pre-treated groups $(p<0.05)$.

Figure 6 shows the effects of SCH 23390 on MA self-administration according to an FR1 schedule of reinforcement. SCH 23390 pre-treatment produced significant changes in responding maintained by MA infusions: $0.01 \mathrm{mg} / \mathrm{kg}$ decreased responding maintained by $0.05 \mathrm{mg} / \mathrm{kg} / \mathrm{infusion} \mathrm{MA}$; whereas the 0.02 $\mathrm{mg} / \mathrm{kg}$ decreased responding maintained by 0.05 and $0.2 \mathrm{mg} / \mathrm{kg} /$ infusion MA. A two-way ANOVA (SCH 23390 x MA) revealed a significant main effect of SCH 
23390 dose $[\mathrm{F}(2,200)=12.45, p=0.0008]$, a main effect of MA dose $[\mathrm{F}(2,200)=$ 6.040, $p=0.00058]$, and an interaction between these factors $[\mathrm{F}(3,200)=5.51, p=$ 0.00031]. Tukey post hoc tests confirmed that $0.02 \mathrm{mg} / \mathrm{kg} \mathrm{SCH} 23390$ decreased MA $(0.05,0.2 \mathrm{mg} / \mathrm{kg} /$ infusion, $p=0.0003)$ maintained responding, and the 0.01 $\mathrm{mg} / \mathrm{kg}$ dose decreased responding for MA $0.05 \mathrm{mg} / \mathrm{kg} /$ infusion $(p=0.003)$. A twoway ANOVA was conducted to clarify the effects of SCH 23390 on active vs. inactive lever responding. Active lever responding was significantly decreased by SCH 23390 pre-treatment $[\mathrm{F}(2,87)=17.23, p=0.0005]$, but inactive lever responding was not significantly affected $[\mathrm{F}(2,87)=2.54, p=0.084]$. 


\subsubsection{Effects of eticlopride}

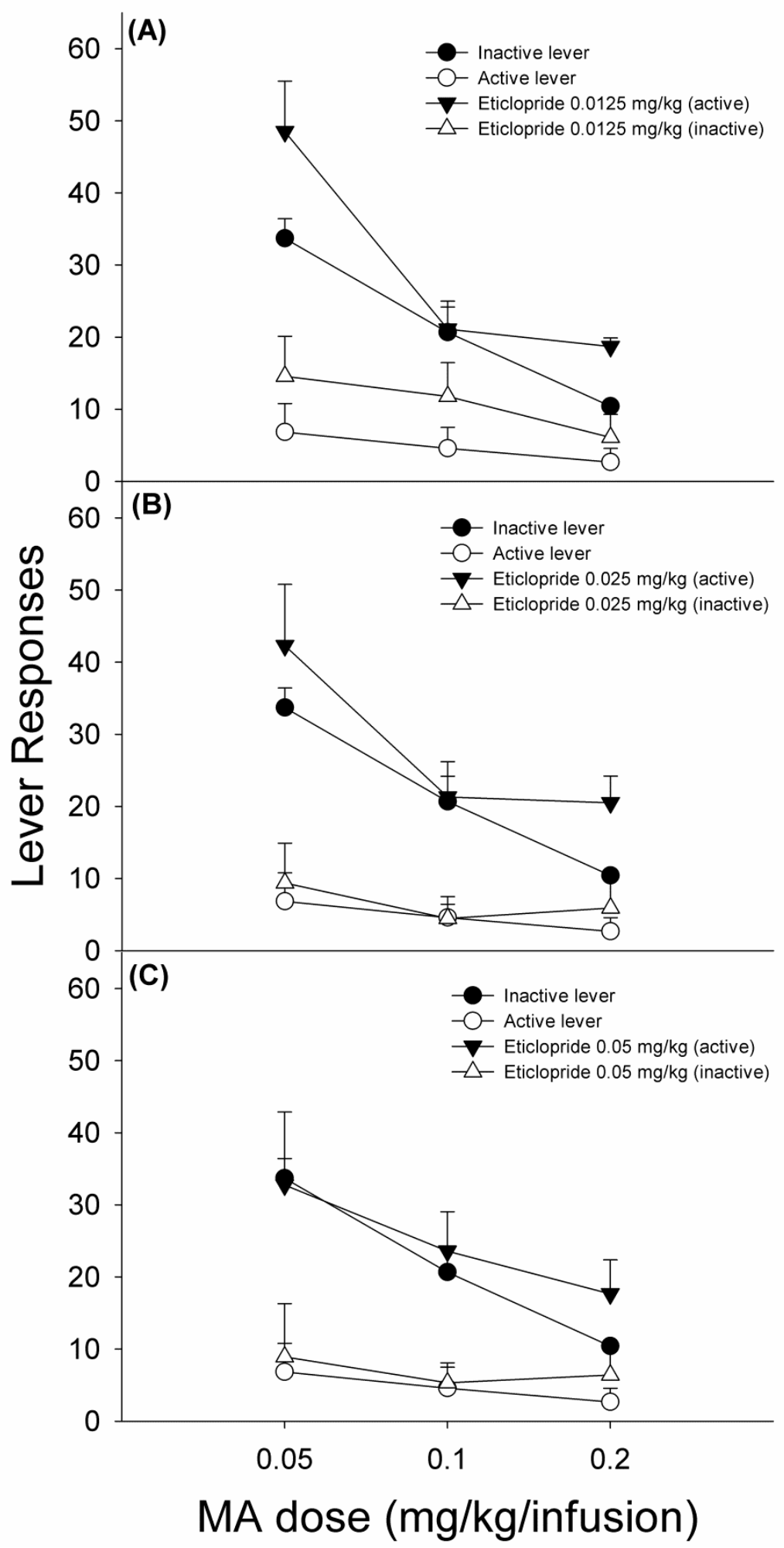

Figure 7. Effects of three eticlopride doses [A) $0.0125 \mathrm{mg} / \mathrm{kg}$; B) $0.025 \mathrm{mg} / \mathrm{kg}$; C) $0.05 \mathrm{mg} / \mathrm{kg}$ ] on lever responding maintained by various doses of MA $(0.05,0.1,0.2 \mathrm{mg} / \mathrm{kg} / \mathrm{inf}$ usion). Symbols represent the mean number of responses (+SEM). 
Figure 7 shows the effects of eticlopride pre-treatment on MA selfadministration reinforced according to an FR1 schedule of reinforcement. Eticlopride pre-treatment failed to significantly alter MA self-administration. ANOVA (eticlopride dose x MA dose) revealed a significant main effect of MA dose $[F(2,299)=11.85, p=0.0023]$, but no significant effect of eticlopride dose $[\mathrm{F}(3,299)=1.21, p=0.307]$, or interaction between MA and eticlopride dose $[\mathrm{F}(5$, $299)=0.45, p>0.847]$. A two-way ANOVA was conducted to investigate effects of eticlopride on active vs. inactive lever responding. Neither active lever $[\mathrm{F}(3,135)=$ $1.18, p=0.320]$, nor inactive lever $[\mathrm{F}(3,135)=0.44, p=0.724]$ responding was significantly affected by eticlopride pre-treatment. There appeared to be a trend toward an increase in responding on both levers across each dose of eticlopride, although this did not reach significance.

\subsection{Responding on the PR schedule of reinforcement}

\subsubsection{Dose-response effects of MA}

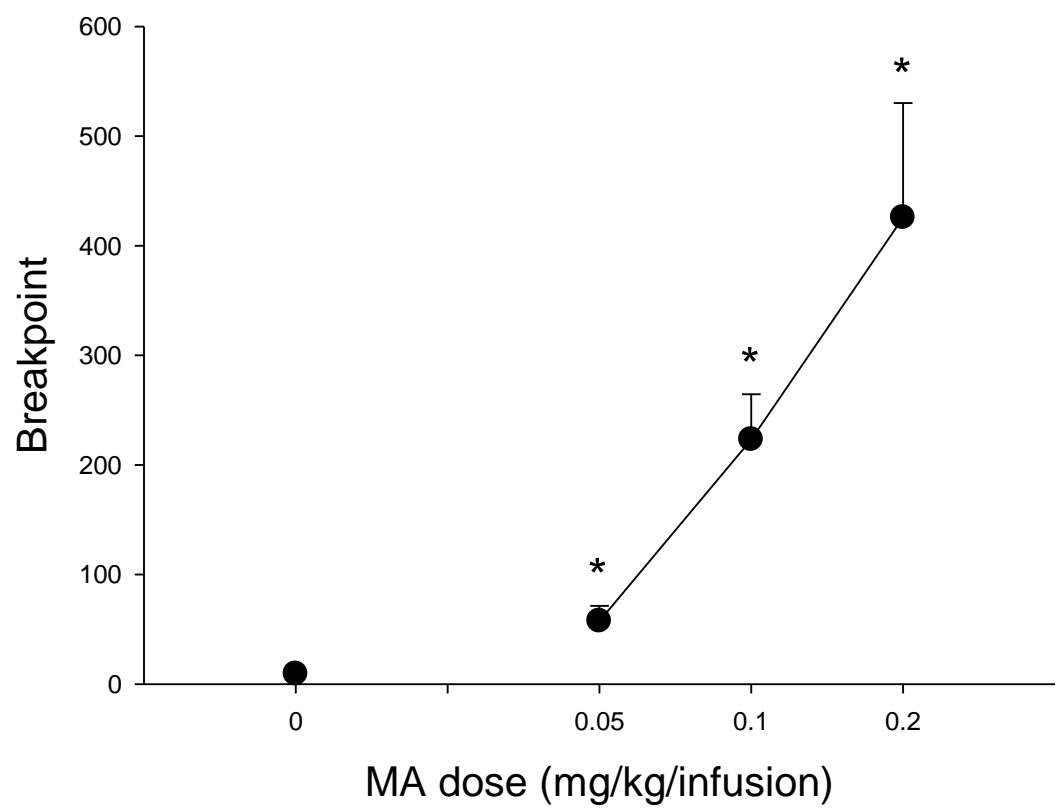

Figure 8. Mean (+SEM) BPs maintained by various doses of MA (0.05, 0.1, $0.2 \mathrm{mg} / \mathrm{kg} / \mathrm{infusion})$. *Significantly different from vehicle maintained BPs $(p<0.05)$.

Figure 8 illustrates the effect of MA dose on BPs reinforced according to a PR schedule of reinforcement. Repeated measures ANOVA revealed that BP increased with increasing dose $[\mathrm{F}(3,27)=20.974, p=0.00032]$, and $t$-tests confirmed that the BP for each MA dose was significantly higher than for vehicle 
infusions $(p=0.008)$. The BP produced by $0.2 \mathrm{mg} / \mathrm{kg} /$ infusion MA was the highest and was used for subsequent testing of the effects of D1- and D2-like antagonists on MA self-administration.

\subsubsection{Effects of SCH 23390}

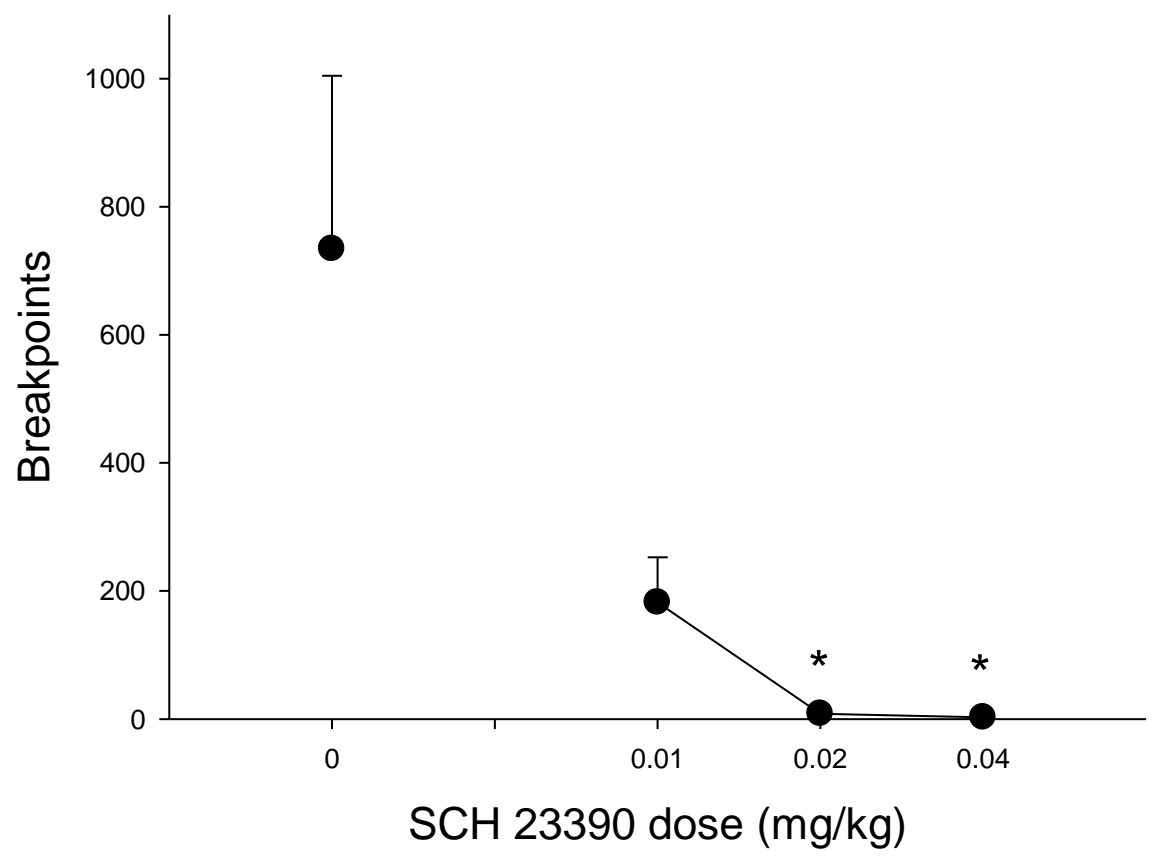

Figure 9. The effect of pre-treatment with various doses of SCH $23390(0.01,0.02,0.04 \mathrm{mg} / \mathrm{kg})$ on mean BPs (+SEM) maintained by MA $(0.2 \mathrm{mg} / \mathrm{kg} /$ infusion). *Significantly different to vehicle pre-treatment $(p<$ 0.05).

Figure 9 shows the effect of SCH 23390 pre-treatment on BPs maintained by $0.2 \mathrm{mg} / \mathrm{kg} /$ infusion MA. ANOVA revealed that $\mathrm{SCH} 23390$ produced a dosedependent decrease in $\mathrm{BP}[\mathrm{F}(3,15)=6.27, p=0.006]$, and post hoc $t$-tests confirmed that the BP was significantly attenuated by the 0.02 and $0.04 \mathrm{mg} / \mathrm{kg}$ doses $(p=0.043)$. 


\subsubsection{Effects of eticlopride}

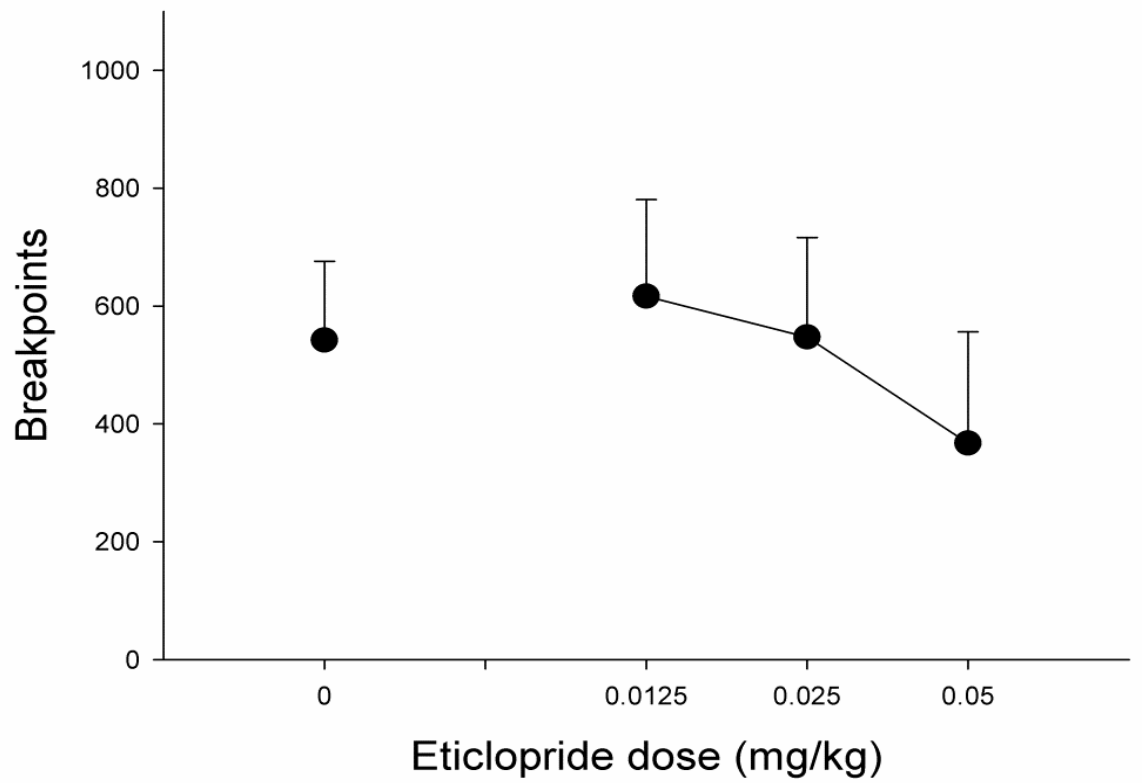

Figure 10. The effect of pre-treatment with various doses of eticlopride $(0.0125,0.025,0.05 \mathrm{mg} / \mathrm{kg})$ on mean BPs (+SEM) maintained by MA $(0.2 \mathrm{mg} / \mathrm{kg} / \mathrm{infusion})$.

Figure 10 depicts the effect of eticlopride pre-treatment on the MA-produced BP. Catheter patency was lost for two of the rats prior to completion of the full doseresponse curve, and therefore, data from these rats were excluded from analysis (final $n=4$ ). Repeated measures ANOVA revealed that there was no significant change in $\mathrm{BP}$ as a function of eticlopride pre-treatment $[\mathrm{F}(3,9)=0.52, p=0.678]$.

\section{Discussion}

Self-administration was produced according to both the FR and PR schedule of reinforcement. Responding under both schedules of reinforcement was dosedependent: rate of responding decreased with increased dose according to the FR schedule; and BPs increased with increased dose according to the PR schedule. These findings reflect typical aspects of MA self-administration under these schedules, and are characteristic of dose manipulations on each schedule (Arnold \& Roberts, 1997; Richardson \& Roberts, 1996; Roberts, et al., 2007). Under FR schedules of reinforcement, rate of responding changes, within certain dose limits, so that a relatively constant level of drug intake is maintained. Accordingly, as the unit dose per infusion is increased, rate of responding decreases in a compensatory manner, characteristic of the descending limb of the dose-response curve. On the 
other hand, under PR schedules, the final ratio completed increases with increasing dose. It has been suggested that the BP reflects a measure of reinforcing efficacy, or incentive motivation for further reinforcement (Arnold \& Roberts, 1997; Hodos, 1961; Richardson \& Roberts, 1996). Hence, as the unit dose per infusion was increased, higher BPs were maintained, suggesting greater reinforcing efficacy and a higher incentive motivation for continued MA reinforcement.

When responding was reinforced according to the FR1 schedule, SCH 23390 pre-treatment produced significant changes in MA self-administration: $0.01 \mathrm{mg} / \mathrm{kg}$ decreased responding maintained by $0.05 \mathrm{mg} / \mathrm{kg} /$ infusion MA; whereas the 0.02 $\mathrm{mg} / \mathrm{kg}$ decreased responding maintained by 0.05 and $0.2 \mathrm{mg} / \mathrm{kg} /$ infusion MA. In contrast, eticlopride pre-treatment failed to significantly alter MA selfadministration at any dose. Similar results were produced when responding was reinforced according to the PR schedule. Under these conditions D1-like receptor blockade produced a reduction in BPs, whereas D2-like receptor blockade had no significant effect. Due to two rats losing catheter patency prior to completing the full dose range of eticlopride pre-treatment, the results from this experiment may be considered preliminary. It is possible that increasing the number of rats in this experiment may have revealed more pronounced effects of eticlopride pre-treatment. Nonetheless, this finding, together with the results from the experiment using the FR1 schedule of reinforcement suggest a preferential role of DA D1-, over D2-like, receptors in the maintenance of MA self-administration, and in the incentive motivation for further MA reinforcement.

Pharmacological blockade of DA receptors often produces non-specific motor impairments that might explain the decrease in MA self-administration (Grace, 2002; Missale, et al., 1998). SCH 23390 pre-treatment, however, did not significantly affect inactive lever responding in the FR experiments. The $0.02 \mathrm{mg} / \mathrm{kg}$ dose of SCH 23390 has previously been shown to increase operant responding reinforced by some doses of both MDMA (Daniela, et al., 2004), and cocaine (Brennan, Lake, et al., 2007) in our laboratory. Furthermore, responding on the PR schedule of reinforcement reached BPs of roughly 200 following pre-treatment with

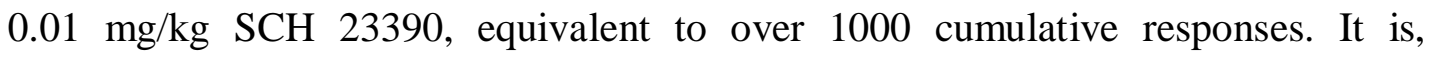
therefore, unlikely that the decrease in responding produced by D1-like receptor blockade reflects an inability to perform the lever press operant. SCH 23390 is also an agonist at the 5-HT2c receptor, which may have affected MA self-administration 
(Munzar, et al., 1999), but the doses required are 10-fold higher than those required for D1-like receptor blockade (Bourne, 2001; Millan, Newman-Tancredi, Quentric, \& Cussac, 2001). The most parsimonious explanation of the data is that D1-like receptors are critical for MA self-administration. The current failure of eticlopride to alter MA self-administration is not likely due to inadequate dosage since MDMA self-administration was increased by these doses when administered under almost identical conditions to those used in the present study (Brennan, et al., 2009). Tests with higher doses of eticlopride were not conducted because they have been shown to produce non-specific generalized decreases in motor activity (Green \& Schenk, 2002; Schenk \& Gittings, 2003).

The effect of SCH 23390 on MA self-administration is consistent with studies that have suggested an important role of this receptor subtype in the selfadministration of other drugs with abuse potential. For instance, pre-treatment with D1-like antagonists altered self-administration of MDMA (Daniela, et al., 2004); heroin (Gerrits, Ramsey, Wolterink, \& Ree, 1994); N-benzylpiperazine (BZP; Brennan, Lake, et al., 2007); methylphenidate (Botly, et al., 2008) and cocaine (Bari \& Pierce, 2005; Barrett, et al., 2004; Caine, et al., 1995; Caine \& Koob, 1994; Hubner \& Moreton, 1991; McGregor \& Roberts, 1995; Ranaldi \& Wise, 2001) on FR schedules of reinforcement. Furthermore, BPs on PR schedules of reinforcement maintained by methylphenidate (Botly, et al., 2008); 'speedball' (cocaine + heroin combination; Cornish, et al., 2005); and cocaine (Bari \& Pierce, 2005; Hubner \& Moreton, 1991; McGregor \& Roberts, 1995; Ranaldi \& Wise, 2001) were reduced following pre-treatment with D1-like antagonists. Together, these findings suggest that D1-like receptor mechanisms are crucial for the self-administration of drugs of abuse, including MA.

The failure of D2-like receptor blockade to decrease MA self-administration is in contrast to other reports of the effects of D2-like receptor antagonists on opiate and psychostimulant self-administration (Botly, et al., 2008; Caine \& Koob, 1994; Caine, et al., 1999; Hubner \& Moreton, 1991; D. Zhang, et al., 2010). The D2 partial agonist $^{2}$ terguride, for example, dose-dependently reduced heroin self-administration on a FR schedule of reinforcement (D. Zhang, et al., 2010) and reduced BPs

${ }^{2}$ Partial agonists bind to DA receptors with high affinity and low intrinsic activity, acting as functional agonists during low dopaminergic tone, and antagonists during high dopaminergic tone, such as during drug self-administration. 
produced by heroin and amphetamine self-administration (Izzo, et al., 2001; D. Zhang, et al., 2010). Eticlopride pre-treatment increased methylphenidate (Botly, et al., 2008) and MDMA self-administration (Brennan, et al., 2009) on a FR schedule of reinforcement, and reduced BPs maintained by methylphenidate selfadministration (Botly, et al., 2008). Pre-treatment with eticlopride also produced rightward shifts in the dose-response function in cocaine self-administration (Barrett, et al., 2004) on an FR schedule of reinforcement. Other D2-like antagonists have produced similar alterations in cocaine self-administration. Spiperone pre-treatment increased responding reinforced by smaller doses on an FR schedule of reinforcement, and decreased responding maintained by higher doses (Hubner \& Moreton, 1991). Self-administration on an FR5 timeout (TO) 20-sec schedule of reinforcement was increased following spiperone pre-treatment, and decreased on an FR15 TO 2-min multiple schedule (Caine \& Koob, 1994).

The experiment using the FR1 schedule of reinforcement enabled a comparison of the effects of the DA antagonists on cocaine and MDMA selfadministration previously investigated in our laboratory. Under these experimental conditions, MA self-administration was preferentially altered by manipulation of D1-like receptor mechanisms, with no significant effect of D2-like manipulations, whereas MDMA (Brennan, et al., 2009; Daniela, et al., 2004) and cocaine (Brennan, Lake, et al., 2007) self-administration were significantly altered by manipulations of both receptor mechanisms. These discrepancies may relate to the divergent consequences of chronic drug exposure on DA neurochemistry and synaptic transmission.

Following a progressively increasing dose regimen of experimenter administered IV infusions of MA, a greater reduction in D2-like receptor binding, compared to D1-like receptor binding, was found in the striatum of rats (Segal, et al., 2005). Similar findings have been reported following self-administered MA exposure. For instance, following approximately 25 daily two hour MA selfadministration sessions, under conditions almost identical to the current study, there were greater decreases in D2-like (21-34\%), over D1-like receptor binding densities (15\%; Stefanski, et al., 1999). A greater effect of D1- over D2-like antagonists, on MA self-administration might, therefore, be expected. Cocaine self-administration under similar protocols did not produce any comparable changes in DA receptor binding densities (Stefanski, et al., 2007), potentially explaining why both D1- and 
D2-like receptor antagonists decreased cocaine self-administration (Botly, et al., 2008; Brennan, Lake, et al., 2007; Caine, et al., 2002). The present results extend the finding of reduced D1- and D2-like receptor binding densities following MA selfadministration (Stefanski, et al., 1999), to suggest a functional disruption in D2-like receptor mediated mechanisms. Further, the current findings show similar effects of D1- and D2-like antagonism when MA self-administration was reinforced according to both the FR and PR schedules. This suggests that these results reflect neuroadaptations as a function of self-administered MA exposure, rather than procedural differences in the schedules of reinforcement. Taken together, these findings suggest that novel neuroadaptations may take place following MA selfadministration, compared to self-administration of other psychostimulants, rendering D2-like receptor mechanisms less responsive to selective pharmacological manipulation.

The present data suggest that following chronic MA self-administration DA D1- and D2-like receptor mechanisms contribute differentially to MA selfadministration, with a greater role of D1- over D2-like mechanisms. This finding is in contrast to the results of previous studies on the effects of D1- and D2-like receptor blockade on drug self-administration, but is consistent with reports on the different neuroadaptations that result from chronic self-administration of other psychostimulants, particularly cocaine. On the basis of these results, it seems that MA self-administration, through novel neuroadaptations not seen following selfadministration of other psychostimulants, becomes preferentially dependent on DA D1-like, over D2-like, receptor mechanisms. It will be of interest to investigate how pharmacological manipulation of these receptor mechanisms influences other behavioural indices of MA self-administration. 


\section{Chapter 3: Role of D1- and D2-like receptor mechanisms in drug-seeking following MA self- administration}

\section{Overview}

Drug abusers reported great difficulty in controlling their drug use despite a strong desire to do so (Cadet, et al., 2003; Julien, 2001), and the high rate of relapse to drug abuse is a critical obstacle to effective long-term treatment (Copeland \& Sorensen, 2001; N. K. Lee, Pohlman, Baker, Ferris, \& Kay-Lambkin, 2010; N. K. Lee \& Rawson, 2008; Shaham, et al., 2003; Shalev, et al., 2002). MA users run a particularly high risk of relapse (Copeland \& Sorensen, 2001) and persistent drug craving can lead to relapse even after long periods of abstinence (Epstein \& Preston, 2003; Epstein, et al., 2006; Hartz, et al., 2001). This emphasizes the importance of research that identifies the relevant neurobiological mechanisms of relapse to MAseeking and MA-taking behaviour.

Self-administration procedures have been effectively used to study reinstatement of drug-seeking and relapse following abstinence. One model in particular is able to determine factors that can reinstate responding following extinguished drug self-administration (de Wit \& Stewart, 1981), such as exposure to the previously self-administered drug, drug-associated stimuli, or stress (Epstein, et al., 2006; Shaham, et al., 2003). Drug-seeking has often been attributed to dopaminergic mechanisms (Green \& Schenk, 2002; Marinelli, Cooper, Baker, \& White, 2003; Schenk, et al., 2011; H. D. Schmidt \& Pierce, 2006; Self, 2004b), as following extinguished drug self-administration, priming injections of DA agonists, including the previously self-administered drug, reinstated drug-seeking behaviour (De Vries, et al., 1999; H. D. Schmidt \& Pierce, 2006; Shaham \& Stewart, 1996; Stewart, 2000). Further, DA D1- and D2-like receptor mechanisms appear critical for this response as priming injections of selective D2 receptor agonists reinstated cocaine- and heroin-seeking (De Vries, et al., 2002; Self, 2004b), and cocaine- and 
heroin-seeking behaviour was attenuated by selective D1- and D2-like receptor antagonists (Self, 1998, 2004b; Self \& Nestler, 1998; Shaham \& Stewart, 1996).

Reinstatement of drug-seeking has also been demonstrated following extinguished MA self-administration (Anggadiredja, et al., 2004; Hiranita, et al., 2010; Moffett \& Goeders, 2007; Rogers, et al., 2008; Shelton \& Beardsley, 2008; Shepard, et al., 2004), but the contribution of DA D1- and D2-like mechanisms to this behaviour have yet to be thoroughly investigated. The finding that pharmacological blockade of DA D1-like receptor mechanisms decreased MA selfadministration on an FR1 and PR schedule of reinforcement (Chapter 2) is consistent with an important role of this receptor in the maintenance of MA abuse and the reinforcing efficacy of MA, and other drugs of abuse (Bari \& Pierce, 2005; Le Merrer, Gavello-Baudy, Galey, \& Cazala, 2007; Ranaldi \& Wise, 2001). Conversely, the lack of effect of a D2-like receptor antagonist on these measures (Chapter 2) is different from the vast body of literature showing a role of D2-like mechanisms in self-administration of other drugs of abuse (Botly, et al., 2008; Caine \& Koob, 1994; Caine, et al., 1999; Hubner \& Moreton, 1991; D. Zhang, et al., 2010). Because the maintenance of MA self-administration was differentially responsive to pharmacological manipulations of D1- and D2-like receptor mechanisms (Chapter 2), it is possible that reinstatement of MA-seeking is also preferentially altered by pharmacological manipulation of D1-like receptor mechanisms. Self-administered MA during two hour sessions produced a greater reduction of DA D2-like receptor densities when measured after 24 drug free hours (Stefanski, et al., 1999), but this effect was transient and the binding measure was comparable to control levels after seven drug free days (Stefanski, et al., 2007). Self-administered MA during six hour sessions, on the other hand, also produced transient reductions in striatal DA levels following 24 hours withdrawal; the deficits were also no longer observed following seven drug free days (Chapter 1). Therefore, the present experiments used two hour self-administration sessions, similar to Stefanski's protocols (1999; 2002), to investigate the contribution of DA D1- and D2-like receptor mechanisms to the reinstatement of extinguished MA-seeking behaviour. This may provide an indication of the functional status of these receptor mechanisms at withdrawal times when receptor densities may be returning to control levels (Stefanski, et al., 2002), in the absence of persistently reduced DA levels (Chapter 1). 
Considering this, the aim of Chapter 3 was to determine the involvement of dopaminergic mechanisms in reinstatement of MA-seeking behaviour. A role of DA was investigated in two ways: by comparing the effects of either a D1- or D2-like receptor antagonist on drug-seeking produced by MA-priming injections; and by comparing the effects of priming injections of a DAT inhibitor, and selective DA D1 and D2 receptor agonists on drug-seeking. The results from Chapter 2 suggest that D1-like receptor mechanisms might play a significant role in reinstatement of MAseeking. Due to the recovery of both D1 and D2 receptor density reductions seven days following MA self-administration (Stefanski, et al., 2002), it was of particular interest to examine the involvement of D2-like receptor mechanisms in this behaviour.

The effects of various doses of experimenter-administered MA on drugseeking were determined in the fifth experiment. Two doses of MA were tested to establish a baseline measure of MA-produced reinstatement of drug-seeking. The dose that generated the highest number of drug-seeking responses was then used for subsequent experiments investigating the selective involvement of D1- and D2-like receptors in relapse to MA-produced drug-seeking behaviour.

In the sixth experiment, the effects of acute pre-treatment with various doses of either the D1-like antagonist, SCH 23390, or the D2-like antagonist, eticlopride, on MA-primed drug-seeking were investigated. For comparison, the effect of pharmacological blockade of D1- and D2-like receptors on MA-seeking produced by cocaine-priming injections was also investigated. Cocaine-primed reinstatement of drug-seeking has previously been attenuated by pre-treatment with both D1- and D2like antagonists (Anderson, et al., 2003; Khroyan, et al., 2000; Schenk \& Gittings, 2003). The purpose of this experiment was, therefore, to determine if there would be any differential effects between MA and cocaine priming-injections and D1- or D2like antagonist pre-treatment, or if a history of MA self-administration had produced neuroadaptations that rendered pre-treatment with the D2-like antagonist ineffective. That is, if the findings between drug primes are similar, this would suggest the effects of D1- or D2-like antagonist pre-treatment are reflective of changes taking place in DA receptor mechanisms as a function of MA self-administration. Furthermore, the effect of eticlopride pre-treatment on MDMA-produced drugseeking in rats experienced with MDMA self-administration was also investigated. Previous research suggests a significant role of D2-like receptor mechanisms in 
relapse to drug-seeking, with D2-like antagonist pre-treatment attenuating drugprimed reinstatement (Khroyan, et al., 2000; Schenk \& Gittings, 2003; Shaham \& Stewart, 1996). This experiment was, therefore, conducted as a positive control of the effects of eticlopride pre-treatment on reinstatement of drug-seeking behaviour following psychostimulant self-administration.

Finally, the seventh experiment further investigated the role of DA in reinstatement of MA-seeking behaviour by measuring drug-seeking following priming injections of various doses of the DAT inhibitor, GBR 12909 (Andersen, 1989), a direct D1 agonist, SKF 81297 (Self, Belluzzi, et al., 1996), and a direct D2 agonist, quinpirole (Malmberg \& Mohell, 1995). In view of previous findings of dopaminergic involvement of drug-seeking following self-administration of other drugs of abuse, it was hypothesized that the indirect DA agonists (MA, cocaine, GBR 12909) would reinstate MA-seeking behaviour. Direct stimulation of D1 receptors has previously failed to potentiate drug-seeking (Alleweireldt, et al., 2002; Khroyan, et al., 2000; Khroyan, et al., 2003; Self, Barnhart, et al., 1996), and blocking both D1- and D2-like receptors attenuated drug-seeking (Bossert, et al., 2007; Green \& Schenk, 2002; Khroyan, et al., 2000; Schenk \& Gittings, 2003; Shaham \& Stewart, 1996), while direct stimulation of D2 receptors has reinstated extinguished drug-seeking behaviour (De Vries, et al., 2002; De Vries, et al., 1999; Khroyan, et al., 2000; Schenk, et al., 2011; Self, Barnhart, et al., 1996; Wise, et al., 1990). Considering the preferential effect of D1-, over D2-like antagonism on maintenance of MA self-administration (Chapter 2), however, it was predicted that D1-, but not D2-like, antagonism would attenuate MA-seeking, while direct stimulation of neither D1- nor D2-like receptor mechanisms would potentiate MAseeking.

\section{Subjects}

Adult, male Sprague-Dawley rats $(n=43)$ were used according to the general methods.

\section{MA \& MDMA Self-administration}

Daily MA self-administration took place as in the general methods. Following at least 10 days of MA self-administration and the fulfilment of 
acquisition criteria, the schedule of reinforcement was increased to FR2 until there was a minimum of three consecutive days of stable responding $(<20 \%$ variation in number of active lever responses), and finally to FR5 for a minimum of 10 days.

Acquisition of MDMA self-administration was conducted during daily six hour sessions. Each depression of the active lever (FR1) produced an infusion of MDMA $1.0 \mathrm{mg} / \mathrm{kg} /$ infusion. Infusion volume and duration remained the same as for MA self-administration, but the $30 \mathrm{sec}$ TO period was omitted. This session-time and dose of MDMA was chosen based on previous studies from our laboratory (Colussi-Mas, Wise, Howard, \& Schenk, 2010; Schenk, Gittings, Johnstone, \& Daniela, 2003; Schenk, et al., 2007). Acquisition criteria for MDMA selfadministration remained the same as for MA. Due to the low responding maintained by this dose of MDMA (Daniela, et al., 2004; Schenk, et al., 2003), the dose was decreased to $0.5 \mathrm{mg} / \mathrm{kg} /$ infusion once the acquisition criteria had been met. Responding was then reinforced according to a FR1 schedule until active lever responding varied by less than $20 \%$ for three consecutive days. Response requirements were then increased to FR2 for a minimum of three days, and then to FR5. The FR5 schedule was maintained for at least five days before tests of drugseeking commenced.

\section{Drug-seeking}

Following stable responding maintained by the FR5 schedule of reinforcement, operant behaviour was extinguished by replacing the drug with a heparinzed ( $3 \mathrm{IU} / \mathrm{ml}$ ) saline solution, and disconnecting the light stimulus that had previously been paired with self-administered drug infusions. The vehicle solution was delivered according to an FR5 schedule. Once the number of active lever presses decreased to $<20 \%$ of baseline, tests of drug-seeking were conducted. On these days, antagonists were administered in the home cage, and agonists were administered in the testing room immediately prior to the start of the test. Responding was reinforced according to an FR5 schedule by the delivery of the vehicle solution and the illumination of the light stimulus, previously paired with self-administered drug infusions during training. The $30 \mathrm{sec}$ TO period was maintained for MA-seeking experiments, but was omitted in MDMA-seeking tests. 


\subsection{Experiment 5: MA-produced drug-seeking}

Initial tests determined the effects of various doses of experimenteradministered MA $(0.0,1.0$, or $2.0 \mathrm{mg} / \mathrm{kg}$, IP, $n=8)$ on drug-seeking behaviour following extinction of MA self-administration. All doses of MA were administered to all rats in a random order. Repeated tests were conducted during a recurring series of drug self-administration (Phase 1; minimum two days), extinction (Phase 2; minimum two days), and reinstatement tests (Phase 3; one day) until all rats had been tested with all three doses of MA.

\subsection{Experiment 6: Effects of D1- and D2-like antagonism on drug- primed drug-seeking}

On the basis of the MA dose-response data obtained in experiment 5, subsequent groups were tested with $2.0 \mathrm{mg} / \mathrm{kg}$ MA. Effects of either the D1-like antagonist, SCH $23390(0.0-0.04 \mathrm{mg} / \mathrm{kg}, \mathrm{SC}, n=5)$, or the D2-like antagonist, eticlopride $(0.0-0.05 \mathrm{mg} / \mathrm{kg}, \mathrm{IP}, n=7)$, on drug-seeking produced by a priming injection of MA (2.0 mg/kg, IP) was measured. Pre-treatments were administered in the home cage 15 (SCH 23390), or 30 (eticlopride) minutes prior to the MA priming-injection. These pre-treatment times and doses were based on previous studies from our laboratory (Brennan, et al., 2009; Brennan, Lake, et al., 2007; Daniela, et al., 2004; Schenk \& Gittings, 2003).

Cocaine-primed MA-seeking tests were conducted to investigate whether the effects of pre-treatment with a D1- or D2-like antagonist were similar to previous studies of cocaine-primed reinstatement of drug-seeking (Anderson, et al., 2003; Khroyan, et al., 2000; Schenk \& Gittings, 2003). Similar effects between drug primes may then suggest DA receptor mechanism changes following MA selfadministration. SCH $23390(n=4)$, and eticlopride $(n=6)$ pre-treatment procedures were identical to those used for MA-primed reinstatement, except that a cocaine (20 $\mathrm{mg} / \mathrm{kg}$, IP) priming-injection was administered immediately prior to placing the rats in the experimental chambers. This dose of cocaine was chosen based on previous studies of drug-seeking from our laboratory (Schenk \& Gittings, 2003; Schenk, et al., 2008). Doses of the antagonists were administered in random order and tests were conducted according to the recurring series outlined above.

MDMA-primed MDMA-seeking tests were conducted as a positive control of the effects of D2-like antagonism on reinstated drug-seeking following selfadministration of another psychostimulant, MDMA. Procedures were identical to the 
other tests on the effects of eticlopride on drug-seeking behaviour, except an MDMA (10.0 mg/kg, IP, $n=5)$ priming-injection was administered immediately prior to placing the rats in the experimental chambers. This dose of MDMA was chosen based on previous studies demonstrating MDMA-seeking in our laboratory (Colussi-Mas, et al., 2010; Schenk, et al., 2008).

\subsection{Experiment 7: DA agonist-primed MA-seeking}

Tests of drug-seeking produced by the DAT inhibitor, GBR 12909 (0.0, 1.0, $10.0 \mathrm{mg} / \mathrm{kg}, \mathrm{IP}, n=4)$, the selective D1 agonist, SKF $81297(0.0-4.0 \mathrm{mg} / \mathrm{kg}, \mathrm{IP}, n=$ 4), and the selective D2 agonist, quinpirole (0.0, $1.0 \mathrm{mg} / \mathrm{kg}, \mathrm{IP}, n=6)$, were measured as above. Drug doses were administered in random order in the experimental room immediately before placing the rat in the experimental chamber. These doses are based on preliminary work and previous studies on the effects of DA agonists on drug-seeking (De Vries, et al., 2002; H. D. Schmidt \& Pierce, 2006; Self, Barnhart, et al., 1996).

\subsection{Drugs}

MDMA hydrochloride was obtained from ESR (Porirua, New Zealand) and was dissolved in a sterile solution of heparinised (3 IU/ml) physiological saline solution for self-administration. GBR 12909 was dissolved in distilled water, while all other drugs (Sigma Aldrich, Australia) were dissolved in a $0.9 \%$ saline solution. Systemic injections were in a volume of $1.0 \mathrm{ml} / \mathrm{kg}$. All drug doses refer to the weight of the salt.

\subsection{Data analysis}

The dose-response curve for MA-primed MA-seeking, effects of the D1- and D2-like antagonists on drug-primed drug-seeking, and drug-seeking produced by the DA agonists were analyzed using separate repeated measures analysis of variance (ANOVA: drug dose x lever). Simple contrasts were used to determine differences between drug doses and vehicle treatment. A number of rats were included in several drug-seeking tests. Extent of MA exposure can influence the magnitude of reinstated MA-seeking (Kitamura, et al., 2006; Rogers, et al., 2008; Shalev, et al., 2002), and, consequently, the ability of DA agonists and antagonists to influence this behaviour. Therefore, separate analyses taking into account the amount of each individual rat's self-administered MA exposure over and above their first drug-seeking test were included in subsequent assays of dopaminergic manipulation of MA-seeking as a 
covariate in analysis of covariance (ANCOVA: drug dose x lever x MA exposure). For illustrative purposes, separate repeated measures ANOVAs only on rats' first reinstatement test (i.e. excluding animals with additional exposure to selfadministered MA, where $n \geq 4$ ), and ANOVAs without MA exposure as a covariate, were also conducted.

\section{Results}

\subsection{Self-administration and extinction responding}

\subsubsection{MA self-administration}

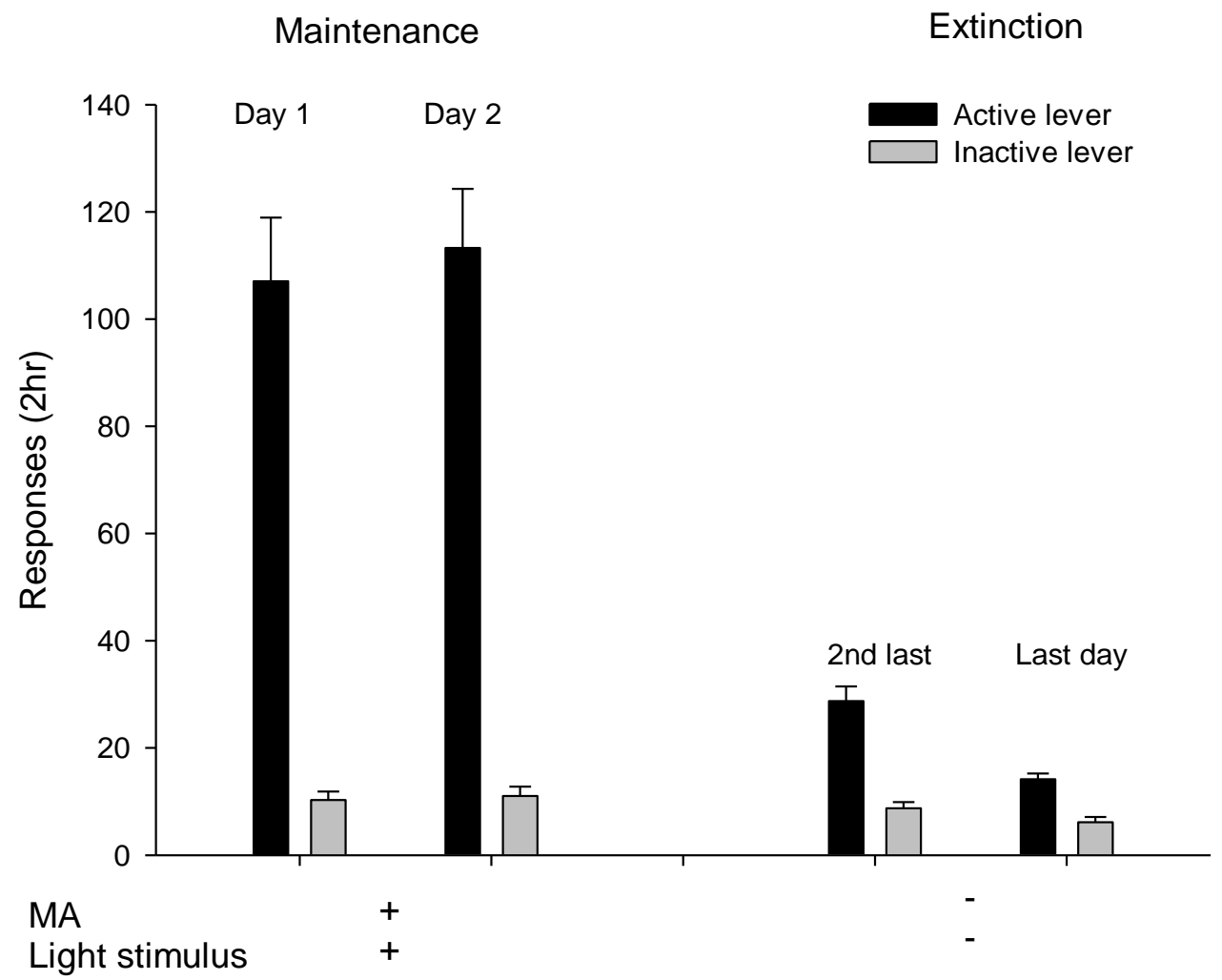

Figure 11. Average (+SEM) responding for MA self-administration on active (black bars), and inactive (grey bars) levers during two days of maintenance (Phase 1), and the last two days of extinction (Phase 2; FR5; $\mathbf{n}=10$ ).

Figure 11 shows the average number of responses on the active and inactive levers during two days of MA self-administration (Phase 1) and the last two days of extinction responding (Phase 2), prior to the start of the reinstatement tests (Phase 3). Responding was high during drug self-administration. When the drug was replaced with vehicle solution and the stimulus light was disconnected, active lever 
responding decreased. The average number of days to reach extinction criteria was $3.3( \pm 0.15)$ days. Repeated measures ANOVA revealed a significant main effect of condition (Phase 1 vs. Phase 2; $[\mathrm{F}(1,9)=38.18, p=0.0002]$ ), lever (active vs. inactive; $[\mathrm{F}(1,9)=51.87, p=0.0005])$, and a significant interaction between these factors $[F(1,9)=32.51, p=0.0002]$. A repeated measures ANOVA was conducted to examine the effect of vehicle priming injections, and the illumination of the stimulus light previously paired with MA-infusions on reinstatement of MA-seeking. Vehicle-priming and the illumination of the previously drug-paired stimulus light reinstated MA-seeking as demonstrated by a main effect of lever $[\mathrm{F}(1,7)=25.75, p$ $=0.001]$, a main effect of condition (Phase 2 vs. Phase $3 ;[F(1,7)=9.15, p=0.019]$, and an interaction between these factors $[\mathrm{F}(1,7)=7.60, p=0.028]$.

\subsubsection{MDMA self-administration}

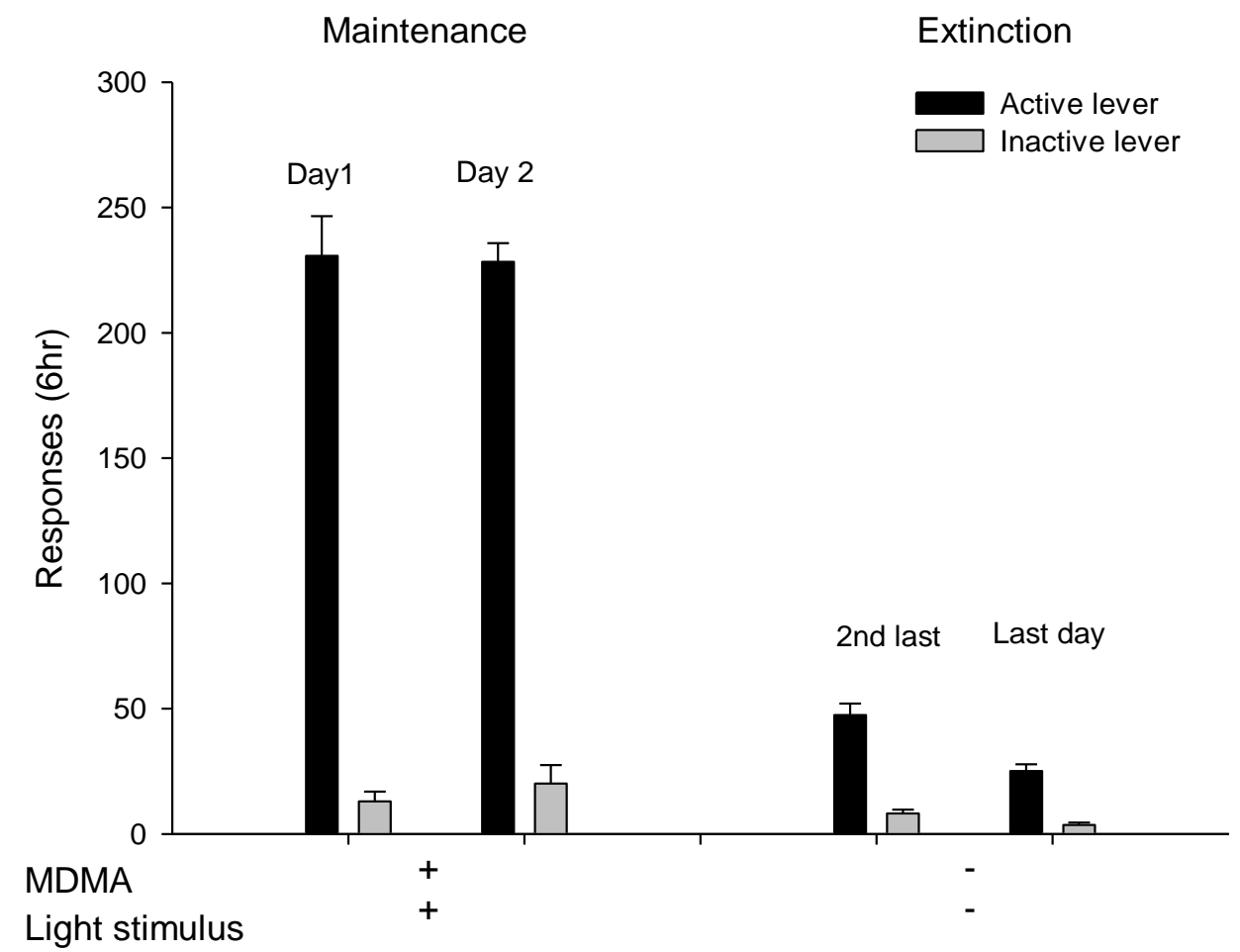

Figure 12. Average (+SEM) responding for MDMA self-administration on active (black bars), and inactive (grey bars) levers during two days of maintenance (Phase 1), and the last two days of extinction (Phase 2; FR5; $\mathbf{n}=10$ ).

Figure 12 shows the average number of responses on active and inactive levers during two days of MDMA self-administration (Phase 1) and the last two days of extinction responding (Phase 2), prior to the start of the reinstatement tests (Phase 3). Responding was high during drug self-administration, and rapidly 
decreased when the drug was replaced with vehicle solution and the stimulus light was omitted. Repeated measures ANOVA revealed a significant main effect of lever $[\mathrm{F}(1,4)=643.20, p=0.0001]$, condition (Phase 1 vs. Phase $2 ;[\mathrm{F}(1,4)=254.85, p=$ $0.00009]$, and an interaction between these factors $[\mathrm{F}(1,4)=335.06, p=0.00005]$.

\subsection{Experiment 5: MA-produced drug-seeking}

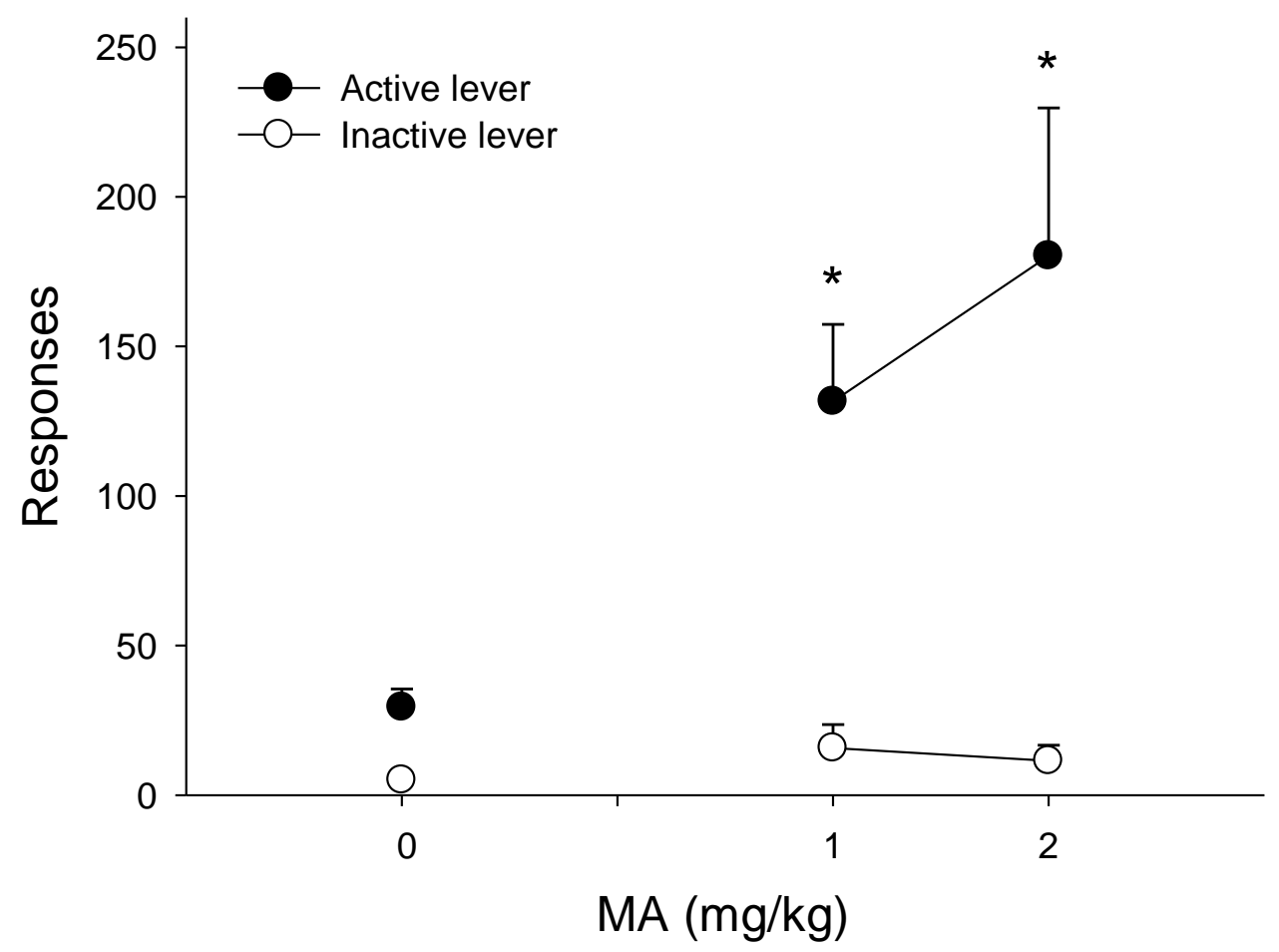

Figure 13. Average responding (+SEM) in drug-seeking tests elicited by various doses of MA $(0 ; 1.0 ; 2.0$ $\mathrm{mg} / \mathrm{kg})$. *Significantly different to vehicle dose $(p<0.05)$

Figure 13 shows the effect of MA $(0.0-2.0 \mathrm{mg} / \mathrm{kg}$, IP) on drug-seeking behaviour. MA-priming injections dose-dependently reinstated previously extinguished drug-seeking behaviour. Repeated measures ANOVA revealed a main effect of lever $[\mathrm{F}(1,7)=34.16, p=0.0006]$, MA-prime $[\mathrm{F}(2,14)=5.509, p=0.017]$ and a significant interaction between MA-prime and lever was observed $[\mathrm{F}(2,14)=$ 4.897, $p<0.024$ ]. Simple contrasts revealed that both doses of the MA-prime significantly increased active lever responding compared to vehicle priming injections $(p=0.025)$. 
32.3 Experiment 6: Effects of D1- and D2-like antagonism on drugprimed drug-seeking

\subsubsection{MA-primed MA-seeking}

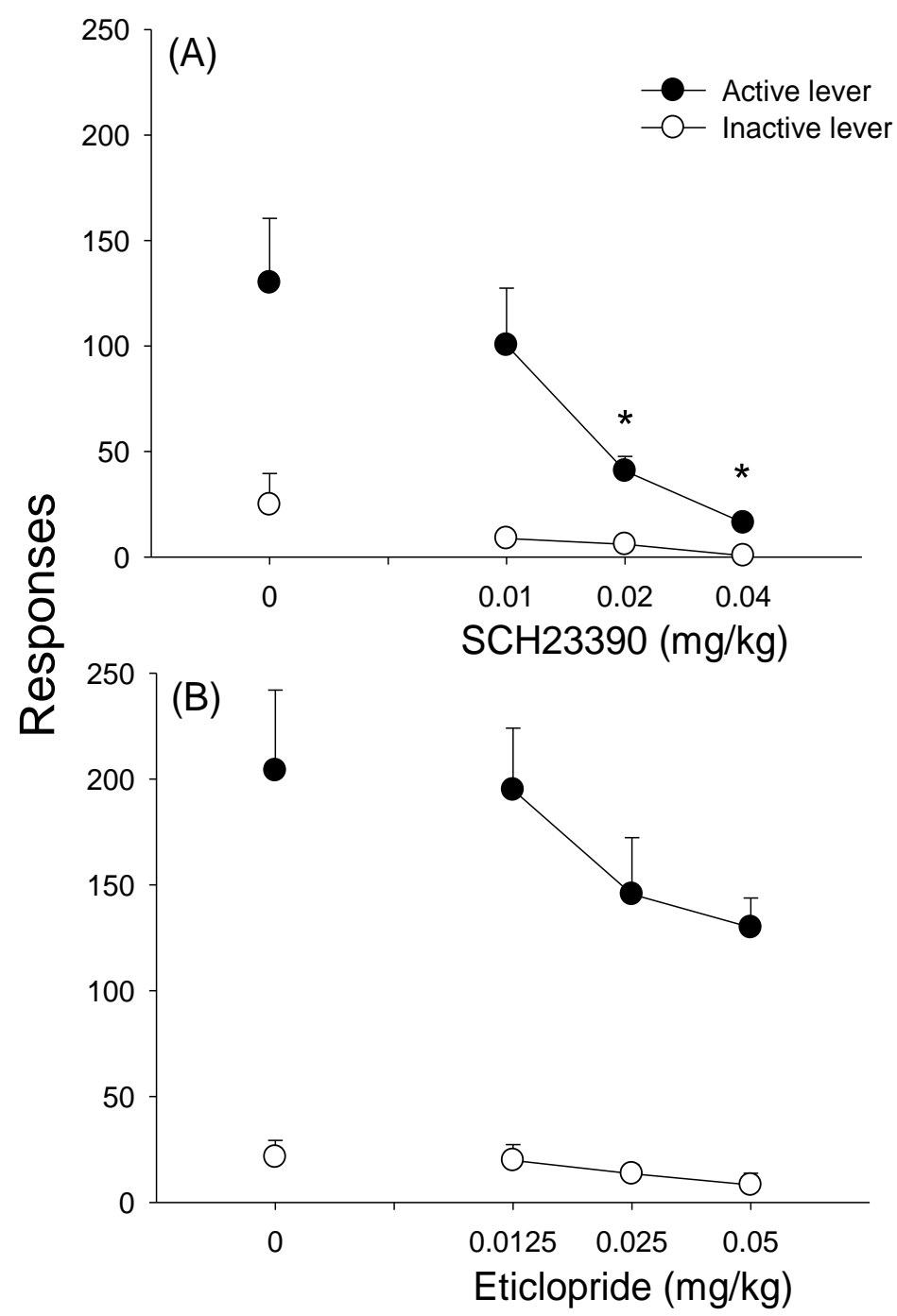

Figure 14. Effects of SCH 23390 [A) $0.0 ; 0.01 ; 0.02 ; 0.04$ ], and eticlopride [B) $0.0 ; 0.0125 ; 0.025 ; 0.05$ $\mathrm{mg} / \mathrm{kg}]$ on drug-seeking produced by MA $(2.0 \mathrm{mg} / \mathrm{kg})$ priming. *Significantly different compared to vehicle $(p<0.05)$

Figure 14 illustrates the effect of pre-treatment with a D1- or D2-like antagonist on drug-seeking produced by MA $(2.0 \mathrm{mg} / \mathrm{kg}$, IP). Pre-treatment with SCH 23390 dose-dependently attenuated MA-produced drug-seeking. Repeated measures ANOVA revealed a main effect of lever $[\mathrm{F}(1,4)=21.77, p=0.01]$, a main effect of SCH 23390 dose $[\mathrm{F}(3,12)=9.15, p=0.002]$, and an interaction between lever and SCH 23390 dose $[F(3,12)=6.71, p=0.007]$. Simple contrasts confirmed 
that the 0.02 and $0.04 \mathrm{mg} / \mathrm{kg}$ doses significantly decreased drug-seeking behaviour $(p=0.0049)$.

Eticlopride did not significantly reduce MA-produced drug-seeking behaviour. A repeated measures ANCOVA, failed to show a significant effect of eticlopride pre-treatment on MA-produced drug-seeking $[\mathrm{F}(3,15)=1.641, p=$ 0.222]. This was corroborated by repeated measures ANOVA on drug-seeking behaviour among rats in their first reinstatement test alone $[\mathrm{F}(3,9)=1.17, p=$ 0.375], and among all rats tested with eticlopride without MA-exposure as a covariate $[\mathrm{F}(3,18)=2.04, p=0.144]$, both showing no significant effect of eticlopride. 


\subsubsection{Cocaine-primed MA-seeking}

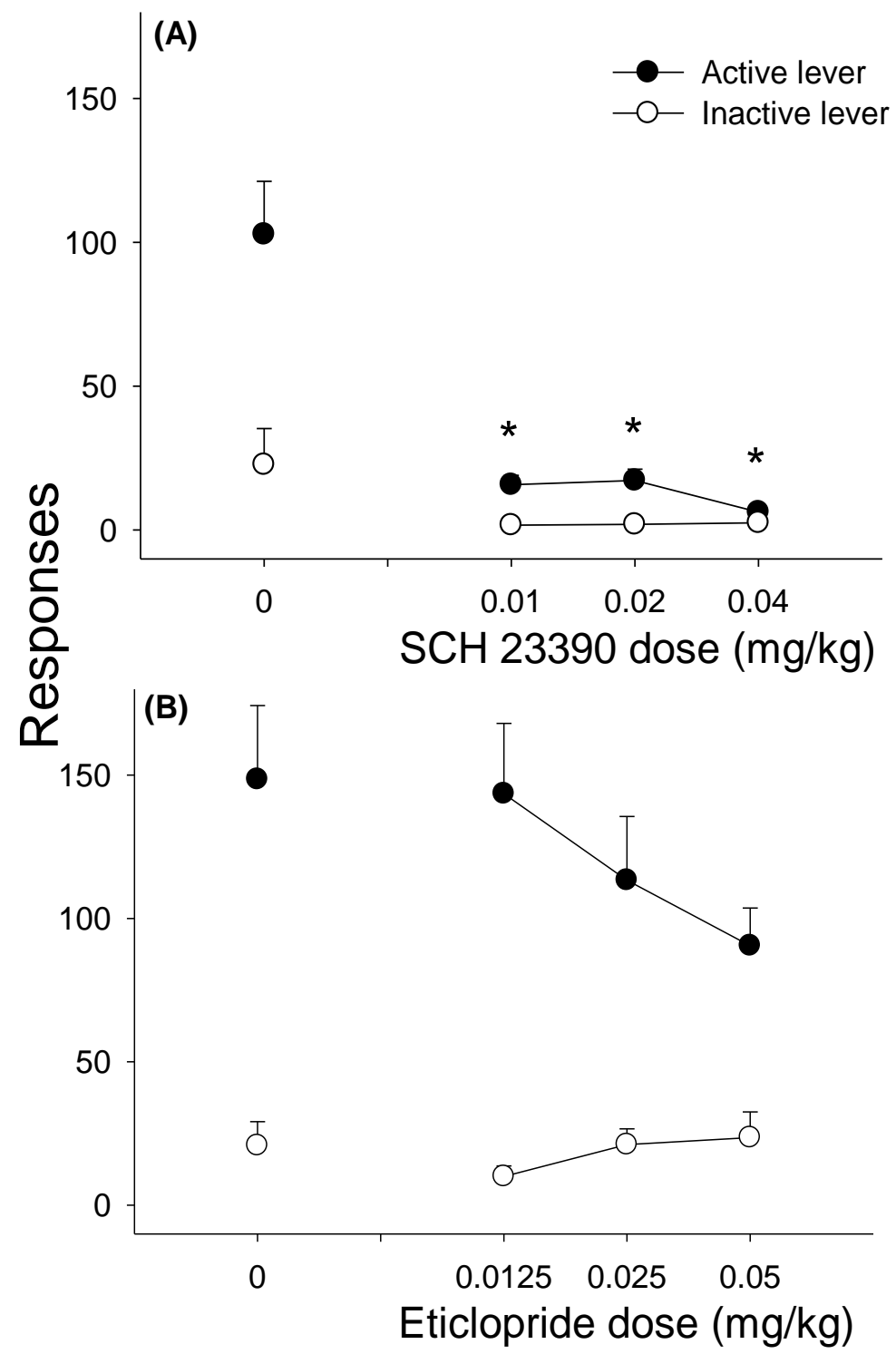

Figure 15. Effects of SCH 23390 [A) $0.0 ; 0.01 ; 0.02 ; 0.04$ ], and eticlopride [B) $0.0 ; 0.0125 ; 0.025 ; 0.05$ $\mathrm{mg} / \mathrm{kg}$ ] on MA-seeking produced by cocaine $(20.0 \mathrm{mg} / \mathrm{kg})$ priming. *Significantly different compared to vehicle $(\mathrm{p}<0.05)$

Figure 15 shows cocaine-produced $(20.0 \mathrm{mg} / \mathrm{kg}$, IP) MA-seeking and the effect of DA D1- and D2-like antagonist pre-treatment on reinstated responding. Pre-treatment with SCH 23390 reduced cocaine-produced MA-seeking. Repeated measures ANCOVA revealed a main effect of lever $[\mathrm{F}(1,2)=18.91, p=0.049]$, $\mathrm{SCH} 23390$ dose $[\mathrm{F}(3,6)=4.91, p=0.047]$, and an interaction between these factors $[\mathrm{F}(3,6)=14.30, p=0.004]$. The effect of D1-like antagonist pre-treatment was supported by repeated measures ANOVA without MA-exposure as a covariate showing a main effect of SCH 23390 pre-treatment $[\mathrm{F}(3,9)=16.44, p=0.001]$. 
Simple contrasts confirmed that all doses of SCH 23390 were effective in reducing MA-seeking behaviour $(p=0.033)$.

Eticlopride failed to reduce cocaine-produced MA-seeking. Repeated measures ANCOVA revealed that pre-treatment with eticlopride did not significantly decrease drug-seeking behaviour $[\mathrm{F}(3,12)=0.46, p=0.188]$, supported by repeated measures ANOVA without MA-exposure as covariate, showing no significant effect of eticlopride $[\mathrm{F}(3,15)=1.689, p=0.212]$.

\subsubsection{MDMA-primed MDMA-seeking}

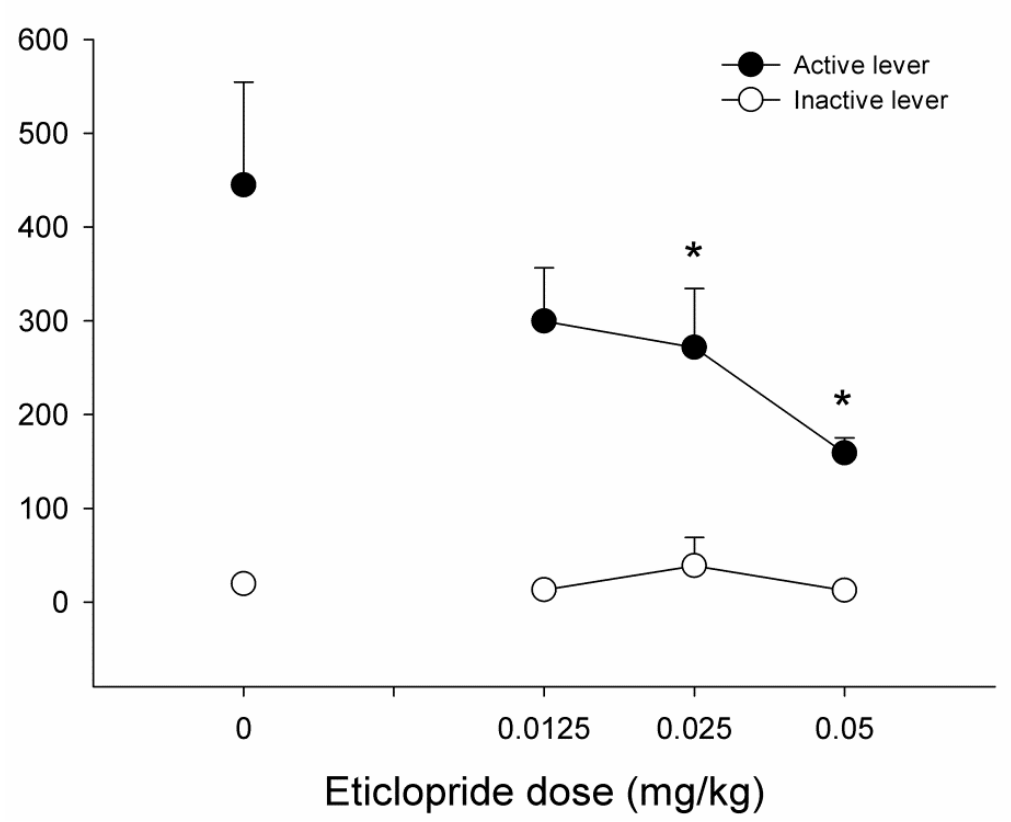

Figure 16. Effect of eticlopride $(0.0 ; 0.0125 ; 0.025 ; 0.05 \mathrm{mg} / \mathrm{kg})$ on drug-seeking produced by MDMA (10.0 $\mathrm{mg} / \mathrm{kg})$ priming.

Figure 16 illustrates the effect of eticlopride pre-treatment on drug-seeking produced by MDMA (10.0 mg/kg, IP) priming-injections. Eticlopride pre-treatment dose-dependently reduced drug-primed MDMA-seeking behaviour. Repeated measures ANOVA exposed a significant main effect of lever $[\mathrm{F}(1,4)=30.10, p=$ $0.006]$, eticlopride dose $[\mathrm{F}(3,12)=3.51, p=0.049]$, and an interaction between these factors $[F(3,12)=4.34, p=0.026]$. Simple contrasts revealed that the two larger doses of eticlopride significantly attenuated MDMA-seeking behaviour ( $p=$ 0.049). 
32.4 Experiment 7: DA agonist-primed MA-seeking

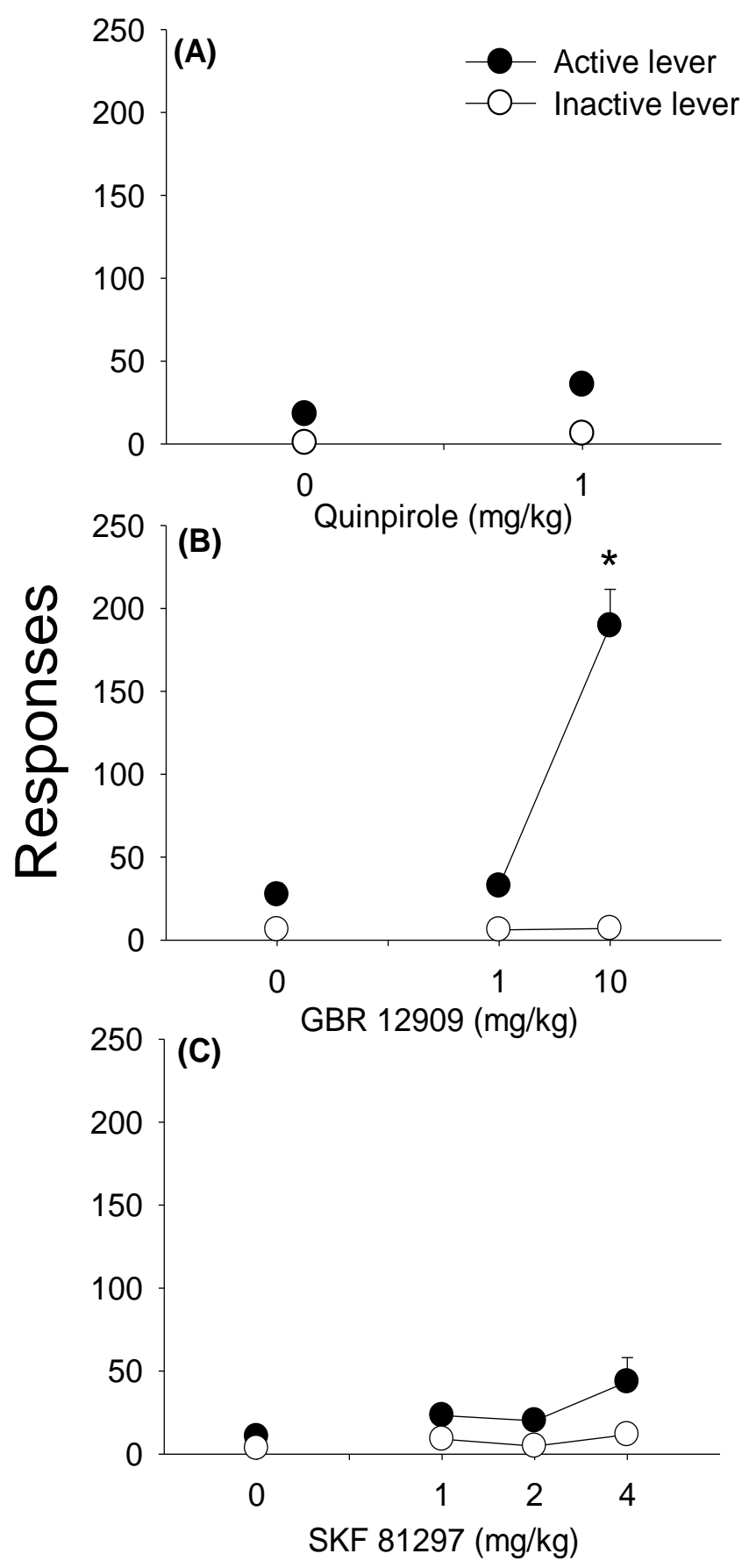

Figure 17. Effect of priming injections of various DA agonists [A) quinpirole $(0.0,1.0 \mathrm{mg} / \mathrm{kg}), \mathrm{B})$ GBR 12909 (0.0, 1.0, 10.0 mg/kg), C) SKF $81297(1.0,2.0,4.0 \mathrm{mg} / \mathrm{kg})]$ on MA-seeking behaviour.

Figure 17 shows the effect of priming-injections of quinpirole (A: 0-1.0 mg/kg, IP), GBR 12909 (B; 0.0-10.0 mg/kg, IP), and SKF 81297 (C; 0.0-4.0 mg/kg, IP) on drug-seeking behaviour. Priming injections of GBR 12909 dose-dependently increased drug-seeking behaviour. Repeated measures ANCOVA revealed a main effect of lever $[\mathrm{F}(1,4)=148.65, p=0.0002]$, a main effect of $\operatorname{GBR} 12909[\mathrm{~F}(2,8)=$ $16.82, p=0.001]$, and an interaction between these factors $[\mathrm{F}(2,8)=27.75, p=$ 
0.0002]. The effect of the GBR 12909 priming-injections was confirmed by repeated measures ANOVA on drug-seeking behaviour among rats in their first reinstatement test alone $[\mathrm{F}(2,6)=14.23, p=0.005]$ and among all rats tested with GBR 12909 without MA-exposure as a covariate $[F(2,10)=29.326, p=0.00006]$. Simple contrasts confirmed that the increase in active lever responding was produced by the $10.0 \mathrm{mg} / \mathrm{kg}$ dose $(p<0.003)$.

Priming-injections of quinpirole did not significantly reinstate MA-seeking behaviour $[\mathrm{F}(1,4)=3.92, p=0.119]$. This was verified by a repeated measures ANOVA on drug-seeking behaviour among rats in their first reinstatement test alone $[\mathrm{F}(1,4)=3.92, p=0.119]$ and among all rats tested with quinpirole without MAexposure as a covariate $[\mathrm{F}(1,5)=5.92, p=0.059]$.

SKF 81297 priming-injections also failed to reinstate MA-seeking behaviour $[\mathrm{F}(3,6)=0.83, p=0.523]$, further supported by a repeated measures ANOVA without MA-exposure as a covariate, also showing no significant effect of SKF $81297[\mathrm{~F}(3,9)=2.81, p=0.1]$.

\section{Discussion}

The results of this study support the idea that dopaminergic mechanisms are important for reinstatement of MA-seeking, and further suggest an important role of D1-, but not D2-like receptor mechanisms. Prior to the first reinstatement test, rats received substantial exposure to self-administered MA during training (32.4-93.9 $\mathrm{mg} / \mathrm{kg}$ over an average of 32 days). When MA was replaced with the vehicle and the drug-paired light stimulus was omitted, responding decreased to less than $20 \%$ of baseline levels. MA-seeking was produced by priming-injections of the DAT inhibitor, GBR 12909, cocaine, and MA indicating an important role for DA in relapse to MA-seeking. However, selective stimulation of DA D1 and D2 receptors by priming-injections of SKF 81297 and quinpirole, respectively, did not reinstate MA-seeking behaviour. Only one dose of quinpirole was tested in the present study and it is possible that a greater dose range may have revealed different effects. The present dose, however, is larger than and equivalent to doses that have reinstated drug-seeking in rats previously self-administering cocaine (De Vries, et al., 2002; De Vries, et al., 1999; Self, Barnhart, et al., 1996), heroin (De Vries, et al., 2002) and MDMA (Schenk, et al., 2011). Dopaminergic mechanisms also appear to play an 
important role in reinstatement of MDMA-seeking behaviour, because; in contrast to MA-seeking, the D2-like antagonist, eticlopride, significantly reduced drug-primed MDMA-seeking. The different findings between the present studies and previous research on the effects of pharmacological manipulation of D2 receptors may be explained by the effects of MA on D2-like receptor mechanisms (Segal, et al., 2005; Stefanski, et al., 1999), discussed in greater detail below.

\subsection{D1- and D2-like antagonist effects on MA-seeking}

SCH 23390 dose-dependently decreased MA-seeking produced by both cocaine and MA priming-injections. A large number of other studies have reported similar effects of SCH 23390, and other D1-like antagonists, consistent with an important role of this receptor in drug-seeking behaviour (Self, 1998, 2004b; Self \& Nestler, 1998; Shaham, et al., 2003; Shalev, et al., 2002). For instance, cocaineseeking produced by stress (foot schock; Capriles, 2003) and drug-associated cues (Alleweireldt, et al., 2002; Bossert, et al., 2007; Liu, et al., 2010) was attenuated by pre-treatment with D1-like antagonists. Heroin- (Bossert, et al., 2007) and nicotineseeking (Liu, et al., 2010) produced by exposure to drug associated cues was also reduced by D1-like antagonist pre-treatment. Cocaine-seeking produced by priming injections of caffeine (Green \& Schenk, 2002), WIN 35,428 (Schenk \& Gittings, 2003), and cocaine (Anderson, et al., 2003; Khroyan, et al., 2000; Schenk \& Gittings, 2003) was reduced by pre-treatment with DA D1-like receptor antagonists. Further, D1-like antagonist pre-treatment also attenuated drug-primed heroin- (Shaham \& Stewart, 1996) and MDMA-seeking behaviour (Schenk, et al., 2011). These findings indicate that, similar to reinstatement of drug-seeking following self-administered exposure to other drugs of abuse, DA D1-like receptor mechanisms appear critical for relapse to MA-seeking behaviour.

The smallest dose of SCH 23390 attenuated cocaine-produced (fig. 15a), but not MA-produced (fig. 14a) drug-seeking. This may relate to the different mechanisms of action of MA and cocaine, their duration of action and subjective effects (Julien, 2001; Krasnova \& Cadet, 2009; Newton, et al., 2005). It has been suggested that reinstatement of drug-seeking is produced by drugs that share discriminative stimulus properties that generalize to the reinforcing effects of the previously self-administered drug (Schenk \& Partridge, 1999; Shaham, et al., 2003; Stewart, 2000). Amphetamine, methylphenidate and caffeine priming-injections, for 
instance, reinstated extinguished cocaine-seeking behaviour (Green \& Schenk, 2002; Schenk \& Partridge, 1999), suggesting they generalized to cocaine's reinforcing effects. Drugs from other pharmacological classes, such as ${ }^{\Delta} 9 \mathrm{THC}$, morphine and nicotine, on the other hand, failed to reinstate cocaine-seeking (Schenk \& Partridge, 1999), suggesting they did not produce discriminative stimulus properties that generalized to cocaine's reinforcing effects. Indeed, GBR 12909 and MA generalized to cocaine in a drug-discrimination task (Holtzman, 2001), and GBR 12909 and cocaine reinstated MA-seeking in the present study, indicating they share discriminative stimulus properties relating to the reinforcing effects of MA. Nonetheless, cocaine and MA have been reported to produce different subjective effects, with MA having a slower onset and longer effects than cocaine (Newton, et al., 2005), and the combined pharmacological mechanism of MA produces a longer elimination half-life (11-12 hours) than that of cocaine (1-2 hours; Julien, 2001). It is, therefore, possible that while cocaine's discriminative stimulus effects generalized sufficiently to reinstate MA-seeking, the more potent effect of the lower dose of SCH 23390 in reducing cocaine-primed drug-seeking may reflect the more rapid peak and decline, and the different subjective effects, compared to the MAprime.

Eticlopride failed to significantly alter the drug-seeking response following the MA- and cocaine-priming injections. Comparisons between cocaine- and MAprimes were made because cocaine-primed drug-seeking has previously been reduced by pre-treatment with both D1- and D2-like antagonists (Anderson, et al., 2003; Khroyan, et al., 2000; Schenk \& Gittings, 2003). It is, therefore, possible that cocaine-priming may have produced differential effects following D1- and D2-like antagonist pre-treatment, compared to the MA-prime. The lack of effect of eticlopride on drug-seeking following both MA- and cocaine-priming injections, however, suggests that this finding reflects changes taking place in DA receptor mechanisms following a history of MA self-administration. This finding is unusual since many other studies have shown an attenuation of drug-seeking behaviour following administration of eticlopride and other D2-like receptor antagonists (Khroyan, et al., 2000; Schenk \& Gittings, 2003; Self, 1998, 2004b; Self, Barnhart, et al., 1996; Shaham \& Stewart, 1996). Cocaine-seeking behaviour in monkeys, for example, was dose-dependently attenuated by pre-treatment with the D2-like antagonists eticlopride and nemonapride (Khroyan, et al., 2000), and in rats by pre- 
treatment with the novel D1 agonist/D2 antagonist LEK-8829 (Milivojevič, et al., 2004). Reinstatement of heroin-seeking behaviour was attenuated by the D2-like antagonist, raclopride, suggesting that D2-like receptors also contribute to heroininduced relapse (Shaham \& Stewart, 1996). Furthermore, following cocaine selfadministration under almost identical protocols as the present study, eticlopride attenuated cocaine-, WIN 35,428- (Schenk \& Gittings, 2003), and caffeine-produced cocaine-seeking (Green \& Schenk, 2002). It is unlikely that the lack of eticlopride effect seen here is due to an inadequate dose as the current doses are comparable to effective doses used in cocaine-primed reinstatement studies (e.g. Khroyan, et al., 2000; Schenk \& Gittings, 2003). Higher doses were not tested because they have been shown to produce non-specific generalized decreases in motor activity (Green \& Schenk, 2002; Schenk \& Gittings, 2003). In addition, pre-treatment with the same doses of eticlopride significantly attenuated MDMA-seeking behaviour in the present study. This suggests that the dose range was behaviourally effective and demonstrates successful attenuation of extinguished drug-seeking behaviour following psychostimulant self-administration with eticlopride.

This lack of effect following D2-like antagonist pre-treatment may relate to the substantial exposure to self-administered MA in the present study, and the consequential neuroadaptations in DA receptor mechanisms following MA selfadministration. Indeed, previous findings showed that 25 days exposure to selfadministered MA produced roughly a two-fold greater reduction in D2-, over D1like receptors (Stefanski, et al., 1999). The present study utilized almost identical protocols to the Stefanksi et al. (1999) study, suggesting that a preferential effect of D1-, over D2-like antagonism might, therefore, be expected. The failure of eticlopride to attenuate both MA- and cocaine-primed reinstatement of MA-seeking is consistent with the finding that eticlopride failed to significantly alter the doseresponse curve for MA self-administration on an FR1 schedule of reinforcement, and reduce BPs on a PR schedule of reinforcement (Chapter 2). Further, these findings suggest that D2-like receptor mechanisms are not critical for the reinstatement of MA-seeking behaviour.

\subsection{DA agonist effects on MA-seeking}

Priming injections of MA, cocaine and the DAT inhibitor, GBR 12909, dosedependently reinstated MA-seeking behaviour, consistent with previous research 
indicating an important role of DA in reinstatement of previously extinguished drugseeking (H. D. Schmidt \& Pierce, 2006; Shaham, et al., 2003; Shalev, et al., 2002). MA is a potent releaser of 5-HT and NE, in addition to DA (Cruickshank \& Dyer, 2009; Julien, 2001; Rothman, et al., 2001), but there is a relative paucity of research into the effects of 5-HT and/or NE agonists on MA-seeking behaviour. The effect of priming-injections of DA, 5-HT, and NE agonists on their ability to reinstate previously extinguished drug-seeking following self-administration of other drugs of abuse, however, has been investigated. A DAT inhibitor (GBR 12909), but not a SERT (fluoxetine) or NET inhibitor (nisoxetine), dose-dependently reinstated cocaine-seeking behaviour, which was subsequently attenuated by pre-treatment with a DA antagonist (H. D. Schmidt \& Pierce, 2006). Reinstatement of heroin- (De Vries, et al., 1999) and MDMA-seeking behaviour (Schenk, et al., 2011) was also induced by priming-injections of GBR 12909. Priming injections of 5-HT receptor agonists (DOI, mCPP), however, failed to reinstate MDMA-seeking, while a SERT inhibitor (clomipramine) attenuated drug-seeking behaviour produced by MDMAassociated cues, suggesting a modulating role for 5-HT in this behaviour (Schenk, et al., 2011). Taken together, these findings indicate a primary role of DA in drugprimed reinstatement of previously extinguished drug-seeking and the present results extend this idea to reinstatement of MA-seeking behaviour.

Priming-injections of the direct D1 agonist, SKF 81297, did not reinstate MA-seeking. These results are supported by previous studies on the effect of direct D1 agonists on reinstatement of previously extinguished responding, suggesting selective stimulation of this receptor did not reinstate drug-seeking behaviour (Self, 2004b; Self \& Nestler, 1998). For instance, priming-injections of the D1 agonists SKF 81297 (Khroyan, et al., 2000), and SKF 82958 (De Vries, et al., 1999; Dias, et al., 2004; Khroyan, et al., 2000; Self, Barnhart, et al., 1996) failed to reinstate cocaine- and heroin-seeking (De Vries, et al., 1999) behaviour. Priming-injections of low doses $(0.3,1.0 \mathrm{mg} / \mathrm{kg})$ of SKF 81297 produced mild reinstating effects on cocaine-seeking in one study, but these effects were eliminated following higher dose $(3.0 \mathrm{mg} / \mathrm{kg}$ ) primes (Alleweireldt, et al., 2002). The dissociation between D1and D2-like receptors on reinstatement of drug-seeking is intriguing as both receptors mediate reinforcing stimuli (Missale, et al., 1998; Self, Belluzzi, et al., 1996; Wise, et al., 1990) and may indicate that D1- and D2-like receptors mediate qualitatively different aspects of drug-seeking. Indeed, it has been suggested that 
D1-like receptors may play a facilitative or 'permissive' role in reinstatement of drug-seeking behaviour (Missale, et al., 1998; Self, 1998, 2004b; Self, Barnhart, et al., 1996; Self \& Nestler, 1998). That is, although selective stimulation of D1-like receptors failed to reinstate drug-seeking (Alleweireldt, et al., 2002; Dias, et al., 2004; Khroyan, et al., 2000; Self, Barnhart, et al., 1996), significant attenuation of reinstated drug-seeking is produced following pre-treatment with D1-like antagonists (Alleweireldt, Kirschner, Blake, \& Neisewander, 2003; Alleweireldt, et al., 2002; Brennan, et al., 2009; Daniela, et al., 2004; Khroyan, et al., 2000; Khroyan, et al., 2003; Schenk \& Gittings, 2003; Shaham \& Miczek, 2003; Shaham, et al., 2003; Shalev, et al., 2002). Thus, because drug-seeking was not produced by direct D1 receptor agonists, but was attenuated by pre-treatment with D1-like antagonists, these findings suggest that stimulation of D1-like receptors alone is insufficient for reinstatement of drug-seeking, but may be necessary in 'permitting' drug-seeking behaviour induced by other stimuli (e.g. exposure to similar drugs, stress, or drugassociated cues). Nonetheless, the doses of SKF 81297 that previously produced mild drug-seeking (Alleweireldt, et al., 2002), did not reinstate MA-seeking in the present study, nor cocaine-seeking in a previous study (Khroyan, et al., 2000). Together, these findings suggest selective stimulation of D1-like receptor mechanisms does not reinstate drug-seeking behaviour (Self, 1998, 2004b; Self \& Nestler, 1998), and the present results extend this idea to reinstatement of MAseeking behaviour.

Priming injections of the direct D2 agonist, quinpirole, failed to reinstate MA-seeking. This finding is atypical because previous studies have reported reinstated drug-seeking behaviour following D2 agonist priming injections in rats (De Vries, et al., 2002; De Vries, et al., 1999; Dias, et al., 2004; Self, Barnhart, et al., 1996; Wise, et al., 1990) and monkeys (Khroyan, et al., 2000), following selfadministration of psychostimulants (De Vries, et al., 2002; De Vries, et al., 1999; Dias, et al., 2004; Khroyan, et al., 2000; Schenk, et al., 2011; Self, Barnhart, et al., 1996; Wise, et al., 1990), and opiates (De Vries, et al., 2002; Wise, et al., 1990). These findings indicate an important role for D2 receptor activation in the reinstatement of drug-seeking behaviour (Self, 1998, 2004a, 2004b; Self \& Nestler, 1998; Shaham, et al., 2003; Shalev, et al., 2002; Stewart, 2000). Quinpirole, specifically, reinstated drug-seeking behaviour in animals previously selfadministering cocaine (De Vries, et al., 2002; De Vries, et al., 1999; Khroyan, et al., 
2000; Self, Barnhart, et al., 1996) and heroin (De Vries, et al., 2002), while the direct D2 agonists bromocriptine and quinelorane reinstated heroin- (Wise, et al., 1990), and cocaine-seeking (Dias, et al., 2004; Wise, et al., 1990), respectively. Only a single dose of quinpirole was tested in the present study, however, and the limitations inherent in using a single dose suggest that testing with higher doses may have produced greater effects. Nonetheless, the dose of quinpirole used in the present study is identical to reinstatement-producing doses in previous studies on cocaine- (De Vries, et al., 2002; Self, Barnhart, et al., 1996), and MDMA-seeking behaviour (Schenk, et al., 2011). The failure of a D2 agonist to induce MA-seeking behaviour is consistent with the finding that a D2-like antagonist failed to alter responding for MA reinforced under both an FR1 and PR schedule (Chapter 2); and MA- and cocaine-primed reinstatement of MA-seeking (above); and lends further support to the hypothesis that D2-like receptor mechanisms undergo neuroadaptations as a function of MA self-administration, rendering them less responsive to pharmacological manipulation, than D1-like receptor mechanisms.

The failure of eticlopride to attenuate, and quinpirole to induce MA-seeking behaviour, respectively, may be explained by the novel effects of repeated MA exposure on DA neurotransmission. Reduced levels of DA and D2-like receptor availability is a consistent finding in human drug abusers, including those with a history of MA abuse (B. Lee, et al., 2009; Martinez, et al., 2009; Volkow, et al., 2001). Rodents self-administering MA for six hours/day presented no changes in monoamine levels in PFC or NAc (Schwendt, et al., 2009), transient reductions in striatal DA levels (Chapter 1), and a persistent decrease in DAT protein levels in PFC and dorsal Str (Schwendt, et al., 2009). Increasing session duration to 15 hours/day, however, produced transient changes in NE and 5-HT levels, with persistent dose-dependent reductions in DA, DAT and TH levels in the Str and cortex (Krasnova, et al., 2010). More extensive testing, using longer duration selfadministration sessions, may, therefore, reveal neurochemical consequences that are not apparent following shorter sessions, as in the current study.

As previously noted, reduced levels of both D1- and D2-like receptor availability have been demonstrated in laboratory animals following experimenteradministered MA infusions, but there were greater reductions in D2- over D1-like receptor binding densities (Segal, et al., 2005). Self-administered MA, under protocols almost identical to the current study, also produced more substantial 
decreases in DA D2-, over D1-like, receptor binding (Stefanski, et al., 1999). Greater reductions in D2-, than D1-like, receptor binding densities, as a function of self-administered MA exposure, may therefore explain the preferential effect of SCH 23390 on MA-seeking behaviour. Comparable effects on DA D2-like receptor binding densities have not always been observed following exposure to other drugs of abuse. Following cocaine exposure, for example, the findings have been somewhat ambiguous, where decreases (Nader, et al., 2002), increases (Peris, et al., 1990), or no change in D2-like receptor binding (D. R. Wallace, Mactutus, \& Booze, 1996) have been reported. Indeed, exposure to self-administered cocaine (2-6 hr sessions) failed to produce persistent changes in D2-like receptor binding densities (Ben-Shahar, et al., 2007; Briand, Flagel, Seeman, \& Robinson, 2008; Laurier, Corrigall, \& George, 1994; Stefanski, et al., 2007). This could explain why drugseeking following cocaine self-administration was susceptible to pharmacological manipulation of both D1- and D2-like receptor mechanisms, whereas drug-seeking following MA self-administration in the present study was only susceptible to pharmacological antagonism of D1-like receptors.

These results are consistent with the idea that MA self-administration may produce neuroadaptations in the dopaminergic system not seen following selfadministration of other drugs of abuse. The findings that eticlopride failed to alter responding for MA self-administration under an FR1 and a PR schedule of reinforcement, together with the present results, suggests that under the current protocols, DA D2-like receptor mechanisms may undergo novel neuroadaptations as a function of self-administered MA exposure, rendering these behaviours more susceptible to pharmacological manipulations of DA D1-like receptor mechanisms.

\section{General discussion}

The aim of this thesis was to understand the neurobiological mechanisms and consequences of MA self-administration, focusing on the dopaminergic system. In particular, a focus was to determine whether there are persistent changes in striatal DA and frontal cortex 5-HT levels following self-administered MA exposure and whether these changes have functional implications for MA self-administration and reinstatement of MA-seeking. These objectives were accomplished in three ways. First, two drug free periods were chosen ( 24 hours vs. seven days) to determine 
whether was recovery of DA and 5-HT levels, their respective metabolites and rates of turnover, following self-administered MA exposure. The effects on contingency on these measures were determined using a yoked-control self-administration protocol. Second, the effects of selective pharmacological manipulation of DA D1and D2-like receptor mechanisms on the maintenance of MA self-administration were determined under an FR1 schedule of reinforcement. For comparison, the effect of these manipulations on changes in the reinforcing efficacy and incentive motivation for further MA reinforcement was determined using a PR schedule of reinforcement. Finally, the contribution of DA and DA D1- and D2-like receptor mechanisms to relapse to MA-seeking behaviour was investigated using the reinstatement paradigm. The current experiments were used to evaluate the above questions and the results can be briefly summarised as follows.

1. Self-administered MA does not produce neurotoxicity in rats, but does produce transient neurochemical disruption and changes in DA regulation.

2. MA exposure produces neuroadaptations predominantly in dopaminergic, rather than serotonergic systems and the striatum appears highly susceptible to MA-produced reductions in tissue levels of DA.

3. These changes likely represent the purely pharmacological effects of exposure to self-administered MA as there were no significant differences between selfadministered MA and passive (yoked) MA infusions on neurochemical measures.

4. DA D1- and D2-like receptor mechanisms contribute differentially to the maintenance of MA self-administration. A greater role of D1- over D2-like mechanisms was suggested since there were significant changes in MA selfadministration maintained by both FR1 and PR schedules of reinforcement following pre-treatment with a selective D1-, but not a selective D2-like receptor antagonist.

5. Similar to other drugs of abuse, dopaminergic mechanisms appear crucial for relapse to MA-seeking. This was suggested by reinstatement of MA-seeking behaviour following priming injections of the indirect DA agonists cocaine, GBR 12909, and MA.

6. DA D1- and D2-like receptor mechanisms contribute differentially to reinstatement of MA-seeking behaviour. MA-seeking was attenuated by pretreatment with a selective D1-like receptor antagonist, but drug-seeking was 
not potentiated by a direct D1 agonist. MA-seeking behaviour was not significantly attenuated by selective D2-like receptor antagonist pre-treatment, and was drug-seeking was not potentiated by priming injections of a selective D2 agonist.

In Chapter 1, a typical experimenter-administered exposure regime produced persistent reductions of DA and 5-HT, in the dorsal Str, and FC, respectively, and transient increases in DA, but not 5-HT, turnover. Similar findings have been produced in other studies utilizing an experimenter-administered MA regime (Friedman, Castañeda, \& Hodge, 1998; Herring, Schaefer, Gudelsky, Vorhees, \& Williams, 2008; Pubill, et al., 2003; Quinton \& Yamamoto, 2006). The effects of chronically self-administered MA on these measures were less pronounced, however, and only transient decreases in striatal DA levels and elevated rates of DA turnover in the dorsal Str were produced. This finding provided an important basis for subsequent experiments by providing a means with which to assess the functional status of DA D1- and D2-like receptors in the absence of substantial and persistent changes in tissue monoamine levels and toxicity. In line with these results, two hour MA self-administration sessions using almost identical parameters to the experiments in Chapters 2 and 3 produced no substantial changes in monoamine neurotransmission or toxicity, but produced greater reductions in D2-, over D1-like receptor densities (Stefanski, et al., 1999; Stefanski, et al., 2002).

Because the rats in the experimenter-administered and self-administering groups were of different ages it was not possible to make direct comparisons between effects of the two exposure regimens. Self-administered MA, however, produced smaller and more transient neurochemical changes. This might reflect differences in susceptibility to neurotoxicity as a function of age (J. E. Hanson, et al., 2009; Imam \& Ali, 2001; Volz, et al., 2009). A test of this possibility would require measuring effects of self-administered MA in younger rats. Regardless, selfadministration is generally considered a valid means of determining effects of drug dependence (Gardner, 2000, 2008; Lynch, et al., 2010; Markou, 2009; Markou, et al., 1993; Panlilio \& Goldberg, 2007; Sanchis-Segura \& Spanagel, 2006), and because self-administered MA failed to produce comparable and persistent deficits in the various neurochemical measures, the data suggest that acute high-dose experimenter-administered MA treatment should not be used to model the effects of chronic MA dependence. 
Other studies have also reported less pronounced and/or less persistent changes in various neurochemical markers following self-administered MA. For example, increased TH mRNA and protein levels were reported 1 day, but not 30 days, following 10 days of MA self-administration (Shepard, et al., 2006). Decreased DAT densities in the striatum and pre-frontal cortex were produced 14 days following the last MA self-administration session, but there were no changes in DA and 5-HT tissue levels, or SERT densities (Schwendt, et al., 2009). Following longer self-administration sessions there were reductions in DA levels (30\%) that persisted for up to 14 days (Krasnova, et al., 2010). Experimenter-administered MA produced greater and more persistent reductions in DA (Friedman, et al., 1998). Taken together with the present results, this suggests that MA self-administration does not produce the extensive neurotoxicity and/or lasting neurochemical disruption that has been observed following high-dose, experimenter-administered treatment regimes (Chapman, et al., 2001; Friedman, et al., 1998).

A small number of studies assessing effects of DA antagonists have suggested that dopaminergic mechanisms underlie the maintenance of MA selfadministration (Higley, et al., 2011; Orio, et al., 2010; Wee, et al., 2007; Yokel \& Wise, 1976). The relative contribution of D1- and D2-like receptor mechanisms, however, has not been thoroughly investigated. Accordingly, Chapter 2 determined effects of selective antagonists on MA self-administration reinforced by an FR1, and a PR schedule of reinforcement. Consistent with findings from studies of cocaine and other drug self-administration (Bari \& Pierce, 2005; Botly, et al., 2008; Brennan, et al., 2009; Caine, et al., 1995; Caine \& Koob, 1994; Cornish, et al., 2005; Hubner \& Moreton, 1991; McGregor \& Roberts, 1995; Phillips, et al., 1994; Ranaldi \& Wise, 2001), D1-like antagonism attenuated MA self-administration. In contrast to the majority of research on effects of DA antagonists on self-administration of other drugs of abuse (Bari \& Pierce, 2005; Botly, et al., 2008; Brennan, et al., 2009; Caine \& Koob, 1994; Caine, et al., 1999; David, et al., 2002; Hubner \& Moreton, 1991; Izzo, et al., 2001; Phillips, et al., 1994; D. Zhang, et al., 2010), the D2-like receptor antagonist, eticlopride, failed to significantly alter MA self-administration. Pretreatment with compounds selective for the D3 receptor, however, have been shown to reduce extended access MA self-administration reinforced according to both an FR and PR schedule. These manipulations, however, had lesser effects following limited access. Pre-treatment with the partial D3 agonist, CJB090, reduced MA self- 
administration reinforced according to an FR1 schedule and lowered BPs during six hour access (Orio, et al., 2010). During one hour access only the highest dose reduced BPs, with no effect on responding reinforced according to an FR1 schedule. Further, pre-treatment with the D3 antagonist, PG01037, only reduced BPs in the extended access group, with no effect in the limited access group (Orio, et al., 2010). This suggests that MA self-administration may be more sensitive to pharmacological manipulation of D2-like receptor mechanisms following extended access. It is, therefore, possible that two hour sessions may have been insufficient to observe the effects of eticlopride pre-treatment in the present study.

Because most studies on the effects of DA antagonists on self-administration make use of simple FR schedules, the contribution of D1- and D2-like receptors to MA self-administration reinforced according to a PR schedule was also assessed. Because BPs on a PR schedule of reinforcement are independent of the rate of responding, the $\mathrm{BP}$ can provide an effective measure of the incentive motivational properties of MA, complementing changes in response rate observed under the FR1 schedule of reinforcement. This greatly increases the convergent validity of any findings and may provide crucial insight into the contribution of DA receptor mechanisms to MA self-administration behaviour, not attainable with either schedule alone. Similar findings were produced regardless of whether the FR1 or PR schedule was applied. Thus, the data suggest that both the positively reinforcing effects and incentive motivational properties of MA are decreased following D1-, but not D2-like receptor blockade.

The findings from Chapter 2 raised the question of how DA D1- and D2-like receptor mechanisms contribute to other indices of MA dependence. The reinstatement paradigm of extinguished drug self-administration is a measure that has been used to show that the same variables that can induce subjective reports of drug craving and provoke relapse to drug dependence in humans (i.e. exposure to the drug of abuse or similar drugs, drug-associated cues and/or stress) also produce reinstatement of drug-seeking in laboratory animals (Epstein \& Preston, 2003; Epstein, et al., 2006; Shaham \& Miczek, 2003; Shaham, et al., 2003; Shalev, et al., 2002; Volkow, et al., 2006). For this reason, Chapter 3 used the reinstatement paradigm to assess the role of dopaminergic mechanisms in relapse to MA dependence, as has been demonstrated following self-administration of other drugs of abuse (Anker \& Carroll, 2010; de Wit \& Stewart, 1981; Dias, et al., 2004; Kalivas 
\& McFarland, 2003; Neisewander, O'Dell, Tran-Nguyen, Castañeda, \& Fuchs, 1996; Schenk, et al., 2011; Shaham, et al., 2003; Stretch \& Gerber, 1973). The D1-like antagonist, SCH 23390, produced a dose-dependent reduction in the magnitude of drug-seeking produced by priming injections of both MA- and cocaine, and priming injections of a direct D1 agonist did not reinstate MA-seeking behaviour. These findings are consistent with previous studies on drug-seeking (Alleweireldt, et al., 2002; Anderson, et al., 2003; Botly, et al., 2008; Khroyan, et al., 2000; Liu, et al., 2010; Milivojevič, et al., 2004; Schenk, et al., 2011; Self, Barnhart, et al., 1996) and have led to the suggestion that D1- like receptor mechanisms may play a facilitative role in the DA mediated effects on relapse to drug-seeking (Missale, et al., 1998; Self, 1998, 2004b; Self \& Nestler, 1998). That is, direct stimuluation of D1 receptors has previoulsy failed to potentiate reinstatement of drug-seeking (De Vries, et al., 1999; Dias, et al., 2004; Khroyan, et al., 2000; Schenk, et al., 2011; Self, Barnhart, et al., 1996), but pre-treatment with D1-like antagonists attenuated drug-seeking behaviour (Alleweireldt, et al., 2003; Alleweireldt, et al., 2002; Brennan, et al., 2009; Daniela, et al., 2004; Khroyan, et al., 2000; Khroyan, et al., 2003; Schenk \& Gittings, 2003; Shaham \& Miczek, 2003; Shaham, et al., 2003; Shalev, et al., 2002). This suggests that direct stimulation of D1 receptors alone does not produce relapse to drug-seeking, but may be a necessary component in relapse produced by exposure to drugs, drug associated cues, or stress. The present findings extend the idea of a central dopaminergic mechanism in reinstatement of drug-seeking to reinstatement of extinguished MA-seeking, and further indicate an important role for D1-like mechanisms in the reinstatement of MA-seeking behaviour.

Pre-treatment with the D2-like antagonist, eticlopride, failed to attenuate MA-seeking produced by priming injections of either MA- or cocaine-priming injections, and the selective D2 agonist, quinpirole, failed to produce drug-seeking. These findings are in contrast to studies on reinstatement of drug-seeking following self-administration of other drugs of abuse. For instance, both psychostimulant- and opiate-seeking was attenuated following pre-treatment with D2-like antagonists (Khroyan, et al., 2000; Schenk \& Gittings, 2003; Self, 1998, 2004b; Self, Barnhart, et al., 1996; Shaham \& Stewart, 1996). Further, drug-seeking behaviour following extinguished cocaine and heroin self-administration was produced following priming injections of direct D2 agonists (De Vries, et al., 2002; De Vries, et al., 1999; Dias, et al., 2004; Khroyan, et al., 2000; Self, Barnhart, et al., 1996; Wise, et al., 1990). 
These findings are consistent with the findings of Chapter 2; D1-like mechanisms play a more significant role than D2-like mechanisms in MA dependence.

A possible explanation for the present findings was that experience with MA self-administration produced neuroadaptations in DA receptor mechanisms that rendered pharmacological manipulation of D2-like receptor mechanisms ineffectual. Because D2-like antagonists have attenuated drug-seeking following cocaine (Green \& Schenk, 2002; Khroyan, et al., 2000; Schenk \& Gittings, 2003), heroin (Shaham \& Stewart, 1996), nicotine (Liu, et al., 2010) and MDMA self-administration in the present study, the effects of cocaine-produced drug-seeking following MA selfadministration allowed for comparisons between priming injections. Cocaineproduced drug-seeking was, however, also resistant to the effects of eticlopride. Rather, the data suggest a functional impairment in the D2-like receptor mediated reinstatement of drug-seeking behaviour as a function of MA self-administration. This idea is further supported by the failure of quinpirole to produce drug-seeking. Together, these findings suggest that D1-like receptor mechanisms are more critical.

MA-seeking produced by MA, cocaine and GBR 12909 suggests that this is a DA mediated behaviour, yet direct stimulation of D1 and D2 receptors did not reinstate drug-seeking. The differential effects of SKF 81297 and the indirect DA agonists that increase synaptic DA may be explained by effects mediated through extrasynaptic D1 receptors. Previous reports suggest that many D1 receptors are located at extra-synaptic sites with a substantial portion of D1 receptor-mediated effects occurring via DA diffusion at sites outside the synapse (Gonon, 1997; Martin-Negrier, Charron, \& Bloch, 2000; Smiley, et al., 1994).The attenuation of drug-seeking following SCH 23390 pre-treatment and the failure of SKF 81297 to potentiate drug-seeking was not surprising, as previous studies have reported similar findings (Alleweireldt, et al., 2003; Alleweireldt, et al., 2002; De Vries, et al., 1999; Khroyan, et al., 2000; Self, Barnhart, et al., 1996), suggesting D1-like agonists and antagonists have similar, rather than opposing effects on relapse (Khroyan, et al., 2000; Khroyan, et al., 2003). Given these findings, it has been suggested that D1and D2-like receptors mediate qualitatively different aspects of drug-seeking, and that selective stimulation of D1-like receptors may produce a tonic firing of DA neurons that 'masks' the D2-like receptor-mediated signal for drug-seeking (Missale, et al., 1998; Self, 1998, 2004b; Self \& Nestler, 1998). Indeed, electrically stimulated DA overflow in striatal neurons was delayed and prolonged by IV administration of 
the D1 agonist, SKF 82958, reduced by SCH 23390 and abolished by 6hydroxydopamine lesions, suggesting this excitation was attributable to D1 receptor stimulation (Gonon, 1997). It is, therefore, possible that the lack of drug-seeking produced by D1-receptor stimulation reflects the extra-synaptic D1 receptor mediated-effects produced by SKF 81297.

The current dose of quinpirole is the same and higher than doses that have previously produced drug-seeking following cocaine (De Vries, et al., 2002; Self, Barnhart, et al., 1996), MDMA (Schenk, et al., 2011) and heroin (De Vries, et al., 2002) self-administration. The limitations inherent in the testing of a single quinpirole dose, however, suggest that use of several, greater doses may have produced greater effects. For instance, a recent study has shown that pre-treatment with a selective DA D3 receptor antagonist, SB-277011A, attenuated MA-primed reinstatement of extinguished MA-seeking (Higley, et al., 2011). Quinpirole also possesses agonist properties at DA D3 receptors (Burris, et al., 1995; Gehlert, Gackenheimer, Seeman, \& Schaus, 1992; Philip Seeman \& Schaus, 1991), with an affinity for D3 receptors sevenfold higher than D2 receptors under conditions that favour receptor coupling with G-proteins (Burris, et al., 1995), such as following psychostimulant exposure (Briand, et al., 2008; P. Seeman, McCormick, \& Kapur, 2007; P. Seeman, Tallerico, Ko, Tenn, \& Kapur, 2002). Testing with higher doses of quinpirole may, therefore, have activated D3 receptors and produced greater drugseeking effects. Differences in the level of MA exposure between the present study and the Higley et al. (2011) study may also explain the significant effects of pharmacological manipulation of D3 receptor mechanisms on MA-seeking. For instance, rats underwent only approximately 14 days of self-administration training prior to extinction conditions (Higley, et al., 2011). MA self-administration training in the present study spanned a minimum of 20 days, with an average of 32 days, and was reinforced by twice the unit dose (MA $0.1 \mathrm{mg} / \mathrm{kg} / \mathrm{infusion}$ ), similar to the protocols that produced a greater reduction in DA D2-, over D1-like receptor binding densities (Stefanski, et al., 1999; 25 days exposure). Half the length of exposure to a dose of self-administered MA half the present unit dose may not have been sufficient to produce the neuroadaptions in dopaminergic receptor mechanisms seen in the Stefanski et al. (1999) study. Additionally, extinction training in the Higley et al. (2011) study was enforced for two weeks, whereas the average extinction period in the present study was $3.3( \pm 0.15)$ days, and five days prior to 
D2 agonist primed testing of reinstatement of MA-seeking. The reductions in D1and D2-like receptor densities (Stefanski, et al., 1999) and striatal DA levels (Chapter 1) following self-administered MA exposure had returned to control levels at seven days withdrawal from MA self-administration (Stefanski, et al., 2002; Chapter 1). These findings suggest that any DA receptor density reductions and functional impairment in these mechanisms produced by MA self-administration in the Higley et al. (2011) study may have recovered at the time of drug-seeking tests, whereas there were still functional impairments in D2 receptor mechanisms at the time of testing in the present study. It would be very interesting to investigate the effects of pre-treatment with a selective D3 antagonist, and direct D3 receptor agonist priming-injections on reinstatement of MA-seeking in future experiments.

The discrepancy between the present findings and self-administration studies with other drugs of abuse may be explained by the different consequences of MA exposure on DA neurochemistry and synaptic transmission. Reduced levels of DA and D2-like receptor density have been consistently reported in humans who abuse drugs, including those with a history of MA abuse (B. Lee, et al., 2009; Martinez, et al., 2009; Martinez, et al., in press; Volkow, et al., 2001). Reduced levels of D2-like receptor availability may explain the lack of significant effect of pharmacological manipulation of these mechanisms on the current behavioural indices. For instance, self-administered MA exposure, under conditions almost identical to the present protocols in Chapters 2 and 3, produced greater decreases in D2-, over D1-like receptor binding densities (Stefanski, et al., 1999). A preferential effect of pharmacological manipulation of D1-, over D2-like receptor mechanisms might, therefore, be expected. Further, the reductions of DA D2-like receptor binding densities (Stefanski, et al., 1999) and striatal DA tissue levels (Chapter 1) observed at 24 hours, had shown recovery to control levels at seven days (Stefanski, et al., 2002; section 1). Extending extinction training in Chapter 3 to seven days would have permitted direct comparison between the functional status of D1- and D2-like receptor mechanisms during reinstatement tests and when striatal DA tissue content levels (Chapter 1), and D1- and D2-like receptor binding densities had returned to control levels (Stefanski, et al., 2002). This may be a benefit to future studies of comparisons between the neurobiological consequences of chronic exposure to selfadministered MA and pharmacological manipulation of the receptor mechanisms involved in MA self-administration and reinstatement of MA-seeking. All tests of 
MA-seeking following selective D2 agonist priming-injections, however, took place following five days of extinction training, close to the time when striatal DA tissue levels (Chapter 1) and D1- and D2-like receptor densities (Stefanski, et al., 2002) had returned to control levels.

Most neuroadaptations, such as those referred to above, revert to control levels within a month or two following cessation of chronic drug self-administration (Krasnova, et al., 2010; Self, 2004b; Stefanski, et al., 2002). The high prevalence of relapse to MA abuse, extending over years (Barlow \& Durand, 2002; Darke, et al., 2008; Hartz, et al., 2001), however, suggests that drug-induced neuroadaptations cannot fully account for the longevity of drug-dependent behavioural changes. It may be possible that even transient changes in receptor density, signalling and gene expression may produce persistent alterations in synaptic transmission and organisation, potentially in learning and memory circuits, which perpetuate the vulnerability to relapse to MA dependence. In this manner, the current results extend previous findings to suggest that close to the observed recovery of DA D1- and D2like receptor densities (Stefanski, et al., 2002), DA tissue levels and DA turnover (Chapter 1); there remain functional impairments in DA D2-like receptor mediation of reinstatement of MA-seeking behaviour.

The lack of significant effect of pharmacological manipulation of DA D2like receptor mechanisms on maintenance of MA self-administration and reinstatement of MA-seeking may have important ramifications for future research into MA dependence. Imaging studies have shown that reduced striatal D2-like receptor availability may be involved in the maintenance of drug-taking and dependence, including that of MA. For instance, compared to control participants with no history of drug dependence, detoxified cocaine abusers (Volkow, et al., 1993) and heroin-dependent participants (Martinez, et al., in press) showed significantly lower levels of striatal DA D2 receptor availability, as measured by PET. PET also showed that lower levels of D2 receptors were associated with a more pleasant response to IV methylphenidate administration in healthy male participants with no history of drug dependence, whereas higher D2 levels predicted more unpleasant subjective effects (Volkow, et al., 1999). Correspondingly, monkeys with lower striatal D2 receptor levels were more likely to self-administer cocaine (Morgan, et al., 2002). These findings suggest D2 receptors have an important influence on the nature of drug taking behaviour and dependence. 
Similar findings have been reported in imaging studies of MA dependence. Indeed, MA-dependent participants were found to have significantly lower levels of D2 receptor availability compared to healthy, non drug-dependent controls (B. Lee, et al., 2009; Volkow, et al., 2001). Furthermore, D2 receptor availability was associated with disrupted metabolism in the orbitofrontal cortex (Volkow, et al., 2001), a region of the brain that has been implicated in the occurrence of compulsive behaviours and the regulation of motivation or "drive" (Volkow \& Fowler, 2000). This suggests that enhanced inappropriate activation of this region secondary to drug-induced DA stimulation may increase the incentive motivation to selfadminister MA to compulsive levels in MA-dependent subjects. Lower levels of D2 receptor availability in MA-dependent subjects was also associated with increased impulsiveness, as measured by the Barratt Impulsiveness Scale (version 11, BIS-II; B. Lee, et al., 2009; Patton, Stanford, \& Barratt, 1995). Impulsivity has been linked with compulsive behaviours, such as drug dependence (Belin, et al., 2008), and MA users exhibit increased impulsivity and impaired decision-making (Cruickshank \& Dyer, 2009), further suggesting that lower levels of D2 receptor availability may mediate compulsive MA-taking (Everitt, et al., 2008; Everitt \& Robbins, 2005).

Whether lower levels of DA D2 receptor availability is a consequence of chronic drug exposure or represents a vulnerability to develop drug dependence remains to be seen. The finding that lower levels of $\mathrm{D} 2$ receptors in participants with no history of drug abuse predicted a pleasant response to psychostimulant administration (Volkow, et al., 1999), suggests that D2 receptors, by regulating pleasant versus unpleasant effects of drug administration, may contribute to the propensity to develop drug dependence. The present findings, together with previous reports of reduced levels of both D1- and D2-like receptor densities following MA self-administration (Stefanski, et al., 1999), on the other hand, suggest that reductions of dopaminergic receptor availability take place as a function of MA selfadministration. These two hypotheses need not be mutually exclusive. It is possible that pre-existing low levels of D2 receptor availability may predispose individuals to develop drug-dependence. Chronic drug self-administration may then exacerbate this predisposition by further reducing D2 receptor availability, contributing to an increase in impulsive temperament, and compulsive drug-taking behaviours that are so indicative of the drug-dependent state (APA, 2000). The functional impairment in DA D2-like receptor mechanisms following MA self-administration observed in the 
present studies, may clarify the increasing rate of MA use, abuse and dependence, relapse to MA-dependence following abstinence, and exceptional difficulty in treating MA-dependence (Cho \& Melega, 2002; Copeland \& Sorensen, 2001; Darke, et al., 2008; Degenhardt, et al., 2008; Hartz, et al., 2001; N. K. Lee \& Rawson, 2008; UNODC, 2010; Wilkins \& Rose, 2004).

The present set of experiments makes a significant contribution to research on the neurobiological mechanisms of MA self-administration by presenting findings of transient disruption in dopaminergic levels and regulation, and a functional impairment in DA D2-like receptor mechanisms produced by selfadministered MA. The results from Chapter 1, showing comparatively mild dopaminergic deficits, provided a basis to infer that changes in MA selfadministration and reinstatement of MA-seeking behaviour following pharmacological manipulation of DA D1- and D2-like receptors were due to functional neuroadaptations in these receptor mechanisms, rather than pervasive dopaminergic deficits or neurotoxicity. Subsequent findings following pre-treatment with DA D1- and D2-like receptor antagonists and agonists showed a differential contribution between these receptor mechanisms, with a greater role of D1- over D2like receptor mechanisms in maintenance of MA self-administration and reinstatement of MA-seeking behaviour. This suggests that MA self-administration may produce dopaminergic neuroadaptations not seen following self-administration of other drugs of abuse, rendering DA D2-like receptor mechanisms functionally impaired, and may help explain the exceptionally addictive nature of MA and increasingly high rates of MA-dependence. 


\section{References}

Adinoff, B. (2004). Neurobiologic processes in drug reward and addiction. Harv Rev Psychiatry, 12(6), 305-320.

Alleweireldt, A. T., Kirschner, K. F., Blake, C. B., \& Neisewander, J. L. (2003). D1-receptor drugs and cocaine-seeking behaviour: investigation of receptor mediation and behavioural disruption in rats. Psychopharmacology (Berl), 168, 109-117.

Alleweireldt, A. T., Weber, S. M., Kirschner, K. F., Bullock, B. L., \& Neisewander, J. L. (2002). Blockade or stimulation of D1 dopamine receptors attenuates cue reinstatement of extinguished cocaine-seeking behavior in rats. Psychopharmacology (Berl), 159, 284-293.

Andersen, P. H. (1989). The dopamine uptake inhibitor GBR 12909: selectivity and molecular mechanism of action. [doi: 10.1016/0014-2999(89)90363-4]. European Journal of Pharmacology, 166(3), 493-504.

Anderson, S. M., Bari, A. A., \& Pierce, R. C. (2003). Administration of the $\mathrm{D}_{1}$-like dopamine receptor antagonist SCH-23390 into the medial nucleus accumbens shell attenuates cocaine priming-induced reinstatement of drug-seeking behaviour in rats. Psychopharmacology (Berl), 168, 132-138.

Anggadiredja, K., Sakimura, K., Hiranita, T., \& Yamamoto, T. (2004). Naltrexone attenuates cue- but not drug-induced methamphetamine seeking: a possible mechanism for the dissociation of primary and secondary reward. Brain Res, 1021, 272-276.

Anglin, M. D., Burke, C., Perrochet, B., Stamper, E., \& Dawud-Noursi, S. (2000). History of the methamphetamine problem. Journal of Psychoactive Drugs, 32(2), 137-141.

Anker, J. J., \& Carroll, M. E. (2010). Reinstatement of cocaine seeking induced by drugs, cues, and stress in adolescent and adults rats. Psychopharmacology (Berl), 208, 211222.

APA. (2000). Diagnostic and Statistical Manual of Mental Disorders (Text Revision, 4th ed.). Washington, DC.: American Psychiatric Association.

Arnold, J. M., \& Roberts, D. C. S. (1997). A Critique of Fixed and Progressive Ratio Schedules Used to Examine the Neural Substrates of Drug Reinforcement. Pharmacology Biochemistry and Behavior, 57(3), 441-447.

Askew, B. M. (1962). Hyperpyrexia as a contributory factor in the toxicity of amphetamine to aggregated mice. British Journal of Pharmacology, 19, 245-257.

Bakit, C., Morgan, M. E., Peat, M. A., \& Gibb, J. W. (1974). Long-term effects of methamphetamine on the synthesis and metabolism of 5-hydroxytryptamine in various regions of the rat brain. Neuropharmacology, 20, 1135-1140.

Bari, A. A., \& Pierce, R. C. (2005). D1-like and D2 dopamine receptor antagonists administered into the shell subregion of the rat nucleus accumbens decrease cocaine, but not food, reinforcement. Neuroscience, 135, 959-968.

Barlow, D. H., \& Durand, V. M. (2002). Abnormal Psychology: An integrative approach. Belmont: Wadsworth.

Barr, A. M., Panenka, W. J., MacEwan, G. W., Thornton, A. E., Lang, D. J., Honer, W. G., et al. (2006). The need for speed: an update on methamphetamine addiction. $J$ Psychiatry Neurosci, 31(5), 301-313.

Barrett, A. C., Miller, J. R., Dohrmann, J. M., \& Caine, S. B. (2004). Effects of dopamine indirect agonists and selective D1-like and D2-like agonists and antagonist on cocaine self-administration and food maintained responding in rats. Neuropharmacology, 47, 256-273. 
Baumann, M. H., Ayestas, M. A., Sharpe, L. G., Lewis, D. B., Rice, K. C., \& Rothman, R. B. (2002). Persistent Antagonism of Methamphetamine-Induced Dopamine Release in Rats Pretreat with GBR12909. The Journal of Pharmacology and Experimental Therapeutics, 301(3), 1190-1197.

Baumann, M. H., Wang, X., \& Rothman, R. B. (2007). 3,4Methylenedioxymethamphetamine (MDMA) neurotoxicity in rats: a reappraisal of past and present findings. Psychopharmacology, 189, 407-424.

Bedford, J. A., Bailey, L. P., \& Wilson, M. C. (1978). Cocaine reinforced progressive ratio performance in the rhesus monkey. Pharmacol Biochem Behav, 9, 631-638.

Belin, D., \& Everitt, B. J. (2008). Cocaine seeking habits depend upon dopamine-dependent serial connectivity linking the ventral with the dorsal striatum. Neuron, 57, 432-441.

Belin, D., Mar, A. C., Dalley, J. W., Robbins, T. W., \& Everitt, B. J. (2008). High impulsivity predicts the switch to compulsive cocaine-taking. Science, 320, 13521355.

Ben-Shahar, O., Keeley, P., Cook, M., Brake, W., Joyce, M., Nyffeler, M., et al. (2007). Changes in levels of D1, D2, or NMDA receptors during withdrawal from brief or extended daily access to IV cocaine. Brain Res, 1131, 220-228.

Bergman, J., Kamien, J. B., \& Spealman, R. (1990). Antagonism of cocaine selfadministration by selective dopamine $\mathrm{D}_{1}$ and $\mathrm{D}_{2}$ antagonists. Behavioural Pharmacology, 1, 355-363.

Berman, S., O'Neill, J., Fears, S., Bartzokis, G., \& London, E. D. (2008). Abuse of amphetamines and structural abnormalities in the brain. Ann. N. Y. Acad. Sci., 1141, 195-220.

Booth, B. M., Leukefeld, C., Falck, R., Wang, J., \& Carlson, R. (2006). Correlates of rural methamphetamine and cocaine users: results from a multistate community study *. Journal of Studies on Alcohol, 67(4), 493(499).

Bossert, J. M., Poles, G. C., Ihbey, K. A., Koya, E., \& Shaham, Y. (2007). Differential Effects of Blockade of Dopamine D1-family Receptors in Nucleus Accumbens Core or Shell on Reinstatement of Heroin Seeking Induced by Contextual and Disrete Cues. The Journal of Neuroscience, 27(46), 12655-12663.

Botly, L. C. P., Burton, C. L., Rizos, Z., \& Fletcher, P. J. (2008). Characterization of methylphenidate self-administration and reinstatement in the rat. Psychopharmacology (Berl), 199, 55-66.

Bourne, J. A. (2001). SCH 23390: The First Selective Dopamine $D_{1}$-Like Receptor Antagonist. CNS Drug Reviews, 7(4), 399-414.

Bradburn, N. M., Rips, L. J., \& Shevill, S. K. (1987). Answering autobiographical questions: the impact of memory and inference on surveys. Science, 236, 157-161.

Brady, A. M., McCallum, S. E., Glick, S. D., \& O'Donnell, P. (2008). Enhanced methamphetamine self-administration in a neurodevelopmental rat model of schizophrenia. Psychopharmacology, 200, 205-215.

Brebner, K., Phelan, R., \& Roberts, D. C. S. (2000). Effect of baclofen on cocaine selfadministration in rats reinforced under fixed-ratio 1 and progressive-ratio schedules. Psychopharmacology (Berl), 148, 314-321.

Brennan, K. A., Carati, C., Lea, R. A., Fitzmaurice, P. S., \& Schenk, S. (2009). Effect of D1 like and D2-like receptor antagonists on methamphetamine and 3,4methylenedioxymethamphetamine self-administration in rats. Behavioural Pharmacology, 20, 688-694.

Brennan, K. A., Johnstone, A., Fitzmaurice, P., Lea, R., \& Schenk, S. (2007). Chronic benzylpiperazine (BZP) exposure produces behavioral sensitization and cross- 
sensitization to methamphetamine (MA). [doi: DOI: 10.1016/j.drugalcdep.2006.10.016]. Drug and Alcohol Dependence, 88(2-3), 204-213. Brennan, K. A., Lake, B., Hely, L. S., Jones, K., Gittings, D., Colussi-Mas, J., et al. (2007). $\mathrm{N}$-benzylpiperazine has characteristics of a drug of abuse. Behavioural Pharmacology, 18, 785-790.

Briand, L. A., Flagel, S. B., Seeman, P., \& Robinson, T. E. (2008). Cocaine selfadministration produces a persistent increase in $\mathrm{D} 2^{\text {High }}$ receptors. European Neuropsychopharmacology, 18, 551-556.

Burris, K. D., Pacheco, M. A., Filtz, T. M., Kung, M.-P., Kung, H. F., \& Molinoff, P. B. (1995). Lack of discrimination by agonists for D2 and D3 dopamine receptors. [doi: 10.1016/0893-133X(94)00099-L]. Neuropsychopharmacology, 12(4), 335-345.

Buxton, J. A., \& Dove, N. A. (2008). The burden and management of crystal meth use. CMAJ, 178(12), 1537-1539.

Cadet, J. L., Jayanthi, S., \& Deng, X. (2003). Speed kills: Cellular and molecular bases of methamphetamine-induced nerve terminal degeneration and neuronal apoptosis. The FASEB Journal, 17, 1775-1788.

Cadet, J. L., Krasnova, I. N., Ladenheim, B., Cai, N., McCoy, M. T., \& Atianjoh, F. E. (2009). Methamphetamine preconditioning: differential protective effects on monoaminergic systems in the rat brain. Neurotox Res, 15, 252-259.

Cadet, J. L., Ladenheim, B., \& Hirata, H. (1998). Effects of toxic doses of methamphetamine (METH) on dopamine D1 receptors in the mouse brain. Brain Res, 786, 240-242.

Caine, S. B., Heinrichs, S. C., Coffin, V. L., \& Koob, G. F. (1995). Effects of the dopamine D-1 antagonist SCH 23390 microinjected into the rat accumbens, amygdala or striatum on cocaine self-administration in the rat. Brain Research, 692, 47-56.

Caine, S. B., \& Koob, G. F. (1994). Effects of dopamine D-1 and D-2 antagonists on cocaine self-administration under different schedules of reinforcement in the rat. The Journal of Pharmacology and Experimental Therapeutics, 270(1), 209-218.

Caine, S. B., Negus, S. S., Mello, N., \& Bergman, J. (1999). Effects of Dopamine D ${ }_{1}$-like and $\mathrm{D}_{2}$-like Agonists in Rats that Self-administer Cocaine. The Journal of Pharmacology and Experimental Therapeutics, 291(1), 353-360.

Caine, S. B., Negus, S. S., Mello, N., Patel, S., Bristow, L., Kulagowski, J., et al. (2002). Role of dopamine D2-like receptors in cocaine self-administration: Studies with D2 receptor mutant mice and novel D2 receptor antagonists. The Journal of Neuroscience, 22(7), 2977-2988.

Caldwell, J., Dring, L. G., \& Williams, R. T. (1972). Metabolism of (14 C) methamphetamine in man, the guinea pig and the rat. Biochem J, 129, 11-22.

Camp, D. M., Browman, K. E., \& Robinson, T. E. (1994). The effects of mehtamphetamine and cocaine on motor behaviour and extracellular dopamine in the ventral striatum of Lewis versus Fischer 344 rats. Brain Res, 668, 180-193.

Capriles, N., Rodaros, D., Sorge, R. E., Stewart, J. (2003). A role for the prefrontal cortex in stress- and cocaine-induced reinstatement of cocaine seeking in rats. Psychopharmacology (Berl), 168, 66-74.

Carlson, N. R. (2004). Physiology of Behaviour (8th ed.). Boston: Allyn and Bacon.

Chang, J., Sawyer, S. F., Lee, R., \& Woodward, D. J. (1994). Electrophysiological and Pharmacological Evidence for the Role of the Nucleus Accumbens in Cocaine Selfadministration in Freely Moving Rats. The Journal of Neuroscience, 14(3), 12241244.

Chapman, D. E., Hanson, G. R., Kesner, R. P., \& Keefe, K. A. (2001). Long-term changes in basal ganglia function after a neurotoxic regimen of methamphetamine. Journal of Pharmacology and Experimental Therapeutics, 296(2), 520-527. 
Cho, A. K., \& Melega, W. P. (2002). Patterns of Methamphetamine Abuse and Their Consequences. Journal of Addictive Diseases, 21(1), 21-34.

Cho, A. K., Melega, W. P., Kuczenski, R., \& Segal, D. S. (2001). Relevance of pharmacokinetic parameters in animal models of methamphetamine abuse. Synapse, $39(2), 161-166$.

Chu, P., Seferian, K. S., Birdsall, E., Truong, J. G., Riordan, J. A., Metcalf, C. S., et al. (2008). Differential regional effects of methamphetamine on dopamine transport. European Journal of Pharmacology, 590, 105-110.

Clemens, K. J., Cornish, J. L., Hunt, G. E., \& McGregor, I. S. (2006). Intravenous methamphetamine self-administration in rats: Effects of intravenous or intraperitoneal MDMA co-administration. Pharmacol Biochem Behav, 85, 454-463.

Collins, R. J., Weeks, J. R., Cooper, M. M., Good, P. I., \& Russell, R. R. (1984). Prediction of abuse liability of drugs using IV self-administration by rats. Psychopharmacology, 82, 6-13.

Colussi-Mas, J., Wise, R. J., Howard, A., \& Schenk, S. (2010). Drug seeking in response to a priming injection of MDMA in rats: relationship to initial sensitivity to selfadministered MDMA and dorsal striatal dopamine. Int J Neuropsychopharmacol., 13(10), 1315-1327.

Copeland, A. L., \& Sorensen, J. L. (2001). Differences between methamphetamine users and cocaine users in treatment. Drug and Alcohol Dependence, 62, 91-95.

Cornish, J. L., Lontos, J. M., Clemens, K. J., \& McGregor, I. S. (2005). Cocaine and heroin ('speedball') self-administration: the involvement of nucleus accumbens dopamine and $\mu$-opiate, but not $\delta$-opiate receptors. Psychopharmacology, 180(1), 21-32.

Cruickshank, C. C., \& Dyer, K. R. (2009). A review of the clinical pharmacology of methamphetamine. Addiction, 104, 1085-1099.

Culbertson, C., De La Garza II, R., Costello, M., \& Newton, T. F. (2009). Unrestricted access to methamphetamine or cocaine in the past is associated with increased current use. Int J Neuropsychopharmacol. , 12(5), 677-685.

Dalley, J. W., Lääne, K., Theobald, D. E. H., Peña, Y., Bruce, C. C., Huszar, A. C., et al. (2007). Enduring deficits in sustained visual attention during withdrawal of intravenous methylenedioxymethamphetamine self-administration in rats: results from a comparative study with $d$-amphetamine and methamphetamine. Neuropsychopharmacology, 32, 1195-1206.

Danaceau, J. P., Deering, C. E., Day, J. E., Smeal, S. J., Johnson-Davis, K. L., Fleckenstein, A. E., et al. (2007). Persistence of tolerance to methamphetamine-induced monoamine deficits. [doi: DOI: 10.1016/j.ejphar.2006.11.045]. European Journal of Pharmacology, 559(1), 46-54.

Daniela, E., Brennan, K. A., Gittings, D., Hely, L., \& Schenk, S. (2004). Effect of SCH 23390 on ( \pm )-3,4-methylenedioxymethamphetamine hyperactivity and selfadministration in rats. Pharmacology, Biochemistry and Behaviour, 77, 745-750.

Darke, S., Kaye, S., McKetin, R., \& Duflou, J. (2008). Major physical and psychological harms of methamphetamine use. Drug and Alcohol Review, 27, 253-262.

David, V., Durkin, T., \& Cazala, P. (2002). Differential effects of the dopamine D2/D3 receptor antagonist sulpiride on self-administration of morphine into the ventral tegmental area or the nucleus accumbens. Psychopharmacology, 160(3), 307-317.

Davidson, C., Lee, T. H., \& Ellinwood, E. H. (2005). Acute and chronic continuous methamphetamine have different long-term behavioral and neurochemical consequences. Neurochemistry International, 46, 189-203. 
De Montis, M. G., Co, C., Dworkin, S. I., \& Smith, J. E. (1998). Modifications of dopamine D1 receptor complex in rats self-administering cocaine. European Journal of Pharmacology, 362, 9-15.

De Vries, T. J., Schoffelmeer, A. N. M., Binnekade, R., Raasø, H., \& Vanderschuren, L. J. M. J. (2002). Relapse to cocaine- and heroin-seeking behaviour mediated by dopamine D2 receptors is time-dependent and associated with behavioural sensitization. Neuropsychopharmacology, 26(1), 18-26.

De Vries, T. J., Schoffelmeer, A. N. M., Binnekade, R., \& Vanderschuren, L. J. M. J. (1999). Dopaminergic mechanisms mediating the incentive to seek cocaine and heroin following long-term withdrawal of IV drug self-administration. Psychopharmacology (Berl), 143, 254-260.

de Wit, H., \& Stewart, J. (1981). Reinstatement of Cocaine-Reinforced Responding in the rat. Psychopharmacology (Berl), 75, 134-143.

Degenhardt, L., Roxburgh, A., Black, E., Bruno, R., Campbell, G., Kinner, S., et al. (2008). The epidemiology of methamphetamine use and harm in Australia. Drug and Alcohol Review, 27, 243-252.

Deneau, G., Yanagita, T., \& Seevers, M. H. (1969). Self-administration of psychoactive substances by the monkey. Psychopharmacology, 16, 30-48.

Derlet, R. W., Rice, P., Horowitz, B. Z., \& Lord, R. V. (1989). Amphetamine toxicity: experience with 127 cases. J Emerg Med, 7, 157-161.

Deroche-Gamonet, V., Belin, D., \& Piazza, P. V. (2004). Evidence for addiction-like behavior in the rat. Science, 305, 1014-1017.

Di Chiara, G. (1999). Drug addiction as dopamine-dependent associative learning disorder. [

]. European Journal of Pharmacology, 375(1-3), 13-30.

Di Chiara, G., \& Bassareo, V. (2007). Reward system and addiction: what dopamine does and doesn't do. Current Opinion in Pharmacology, 7, 69-76.

Di Chiara, G., Bassareo, V., Fenu, S., De Luca, M. A., Spina, L., Cadoni, C., et al. (2004). Dopamine and drug addiction: the nucleus accumbens shell connection. Neuropharmacology, 47, 227-241.

Di Chiara, G., \& Imperato, A. (1988). Drugs abused by humans preferentially increase synaptic dopamine concentrations in the mesolimbic system of freely moving rats. Proc. Natl. Acad. Sci. USA, 85, 5274-5278.

Dias, C., Lachize, S., Boilet, V., Huitelec, E., \& Cador, M. (2004). Differential effects of dopaminergic agents on locomotor sensitisation and on the reinstatement of cocaineseeking and food-seeking behaviour. . Psychopharmacology, 175, 414-427.

Downs, D. A., \& Woods, J. H. (1974). Codeine- and cocaine-reinforced responding in rhesus monkeys: effect of dose on response rates under a fixed-ratio schedule. The Journal of Pharmacology and Experimental Therapeutics, 191(1), 179-188.

Dworkin, S. I., Co, C., \& Smith, J. E. (1995). Rat brain neurotransmitter turnover rates altered during withdrawal from chronic cocaine administration. Brain Research, 682, 116-126.

Dwoskin, L. P., \& Crooks, P. A. (2002). A novel mechanism of action and potential use for lobeline as a treatment for psychostimulant abuse. Biochemical Pharmacology, 63, 89-98.

Eddy, N. B., Hallbach, H., Isbell, H., \& Seevers, M. H. (1965). Drug dependence: its siginificance and characteristics. Bull. World Health Organ., 32, 721-733.

Ehrman, R. N., Robbins, S. J., \& Cornish, J. W. (1997). Comparing Self-reported Cocaine Use With Repeated Urine Tests in Outpatient Cocaine Abusers. Experimental and Clinical Psychopharmacology, 5(2), 150-156. 
Eisch, A. J., Schmued, L. C., \& Marshall, J. F. (1998). Characterizing cortical neuron injury with fluoro-jade labeling after a neurotoxic regimen of methamphetamine. Synapse, 30(3), 329-333.

Eisinger, G. J., Wodarski, J. S., \& Ferguson, D. (2009). Psychosocial Correlates of Methamphetamine Use. Journal of Human Behavior in the Social Environment, 19(7), 915 - 931.

Elkashef, A., Vocci, F., Hanson, G., White, J., Wickes, W., Tiihonen, J. (2008). Pharmacotherapy of Methamphetamine Addiction: An Update. Substance Abuse, 29(3), 31-49.

Epstein, D. H., \& Preston, K. L. (2003). The reinstatement model and relapse prevention: a clinical perspective. Psychopharmacology (Berl), 168, 31-41.

Epstein, D. H., Preston, K. L., Stewart, J., \& Shaham, Y. (2006). Toward a model of drug relapse: an assessment of the validity of the reinstatement procedure. Psychopharmacology, 189, 1-16.

Everitt, B. J., Belin, D., Economidou, D., Pelloux, Y., Dalley, J. W., \& Robbins, T. W. (2008). Neural mechanisms underlying the vulnerability to develop compulsive drugseeking habits and addiction. Philosophical Transactions of the Royal Society of Biological Sciences, 363, 3125-3135.

Everitt, B. J., \& Robbins, T. W. (2005). Neural systems of reinforcement for drug addiction: from actions to habits to compulsion. Nature Neuroscience, 8(11), 1481-1489.

Feltenstein, M. W., \& See, R. E. (2008). The neurocircuitry of addiction: an overview. British Journal of Pharmacology, 154, 261-274.

Fischer, J. F., \& Cho, A. (1979). Chemical release of dopamine from striatal homogenates: Evidence for an exchange diffusion model. The Journal of Pharmacology and Experimental Therapeutics, 208(2), 203-209.

Fischman, M. W., \& Schuster, C. R. (1974). Tolerance development to chronic methamphetamine intoxication in the Rhesus monkey. [doi: 10.1016/00913057(74)90010-0]. Pharmacology Biochemistry and Behavior, 2(4), 503-508.

Fleckenstein, A. E., Gibb, J. W., \& Hanson, G. R. (2000). Differential effects of stimulants on monoaminergic transporters: Pharmacological consequences and implications for neurotoxicity. European Journal of Pharmacology, 406, 1-13.

Fleckenstein, A. E., Volz, T. J., Riddle, E. L., Gibb, J. W., \& Hanson, G. R. (2007). New insights into the mechanism of action of amphetamines. Annu. Rev. Pharmacol. Toxicol., 47, 681-698.

Fletcher, P. J. (1998). A comparison of the effects of risperidone, raclopride and ritanserin on intravenous self-administration of $d$-amphetamine. Pharmacol Biochem Behav, 60(1), 55-60.

Friedman, S. D., Castañeda, E., \& Hodge, G. K. (1998). Long-term monoamine depletion, differential recovery, and subtle behavioral impairment following methamphetamineinduced neurotoxicity. Pharmacol Biochem Behav, 61(1), 35-44.

Fuller, R. W., \& Hemrick-Leuke, S. K. (1980). Long-lasting depletion of striatal dopamine by a single injection of amphetamine in inprindole-treated rats. Science, 209, 305307.

Gardner, E. L. (2000). What we have learned about addiction from animal models of drug self-administration. The American Journal on Addictions, 9, 285-313.

Gardner, E. L. (2008). Use of animal models to develop antiaddiction medications. Curr Psychiatry Rep., 10(5), 377-384.

Gehlert, D. R., Gackenheimer, S. L., Seeman, P., \& Schaus, J. (1992). Autoradiographic localization of $[3 \mathrm{H}]$ quinpirole binding to dopamine D2 and D3 receptors in rat brain. 
[doi: 10.1016/0014-2999(92)90528-C]. European Journal of Pharmacology, 211(2), 189-194.

Gerrits, M., Ramsey, N., Wolterink, G., \& Ree, J. (1994). Lack of evidence for an involvement of nucleus accumbens dopamine D1 receptors in the initiation of heroin self-administration in the rat. Psychopharmacology, 114(3), 486-494.

Goldberg, S. R. (1973). Comparable behaviour maintained under fixed-ratio and secondorder schedules of food presentation, cocaine injection or $d$-amphetamine injection in the squirrel monkey. The Journal of Pharmacology and Experimental Therapeutics, 186(1), 18-30.

Gonon, F. (1997). Prolonged and extrasynaptic excitatory action of dopamine mediated by D1 receptors in the rat striatum in vivo. The Journal of Neuroscience, 17(15), 59725978.

Gonzales, R., Mooney, L., \& Rawson, R. A. (2010). The methamphetamine problem in the United States. Annu. Rev. Public Health, 31(385-398).

Grace, A. A. (Ed.). (2002). Dopamine.

Graham, D. L., Noailles, P.-A. H., \& Cadet, J. L. (2008). Differential neurochemical consequences of an escalating dose-binge regimen followed by single-day multipledose methamphetamine challenges. Journal of Neurochemistry, 105(5), 1873-1885.

Grech, D., Spealman, R., \& Bergman, J. (1996). Self-administration of $\mathrm{D}_{1}$ receptor agonists; receptor agonists by squirrel monkeys. Psychopharmacology, 125(2), 97-104.

Green, T. A., \& Schenk, S. (2002). Dopaminergic mechanism for caffeine-produced cocaine seeking in rats. Neuropsychopharmacology, 26, 422-430.

Griffiths, R. R., Bradford, L. D., \& Brady, J. V. (1979). Progressive ratio and fixed ratio schedules of cocaine-maintained responding in baboons. Psychopharmacology, 65, 125-136.

Hamamura, T., Akiyama, K., Akimoto, K., Kashihara, K., Okumura, K., Ujike, H., et al. (1991). Co-administration of either a selective $\mathrm{D}_{1}$ or $\mathrm{D}_{2}$ dopamine antagonist with methamphetamine prevents methamphetamine-induced behavioural sensitization and neurochemical change, studied by in vivo intracerebral dialysis. Brain Research, 546, 40-46.

Hanson, G. R., \& Fleckenstein, A. E. (2009). Basic neuropharmacological mechanisms of methamphetamine. In G. R. Hanson (Ed.), Methamphetamine addiction: From basic science to treatment (pp. 30-60): Guilford Press.

Hanson, J. E., Birdsall, E., Seferian, K. S., Crosby, M. A., Keefe, K. A., Gibb, J. W., et al. (2009). Methamphetamine-induced dopaminergic deficits and refractoriness to subsequent treatment. European Journal of Pharmacology, 607, 68-73.

Harrod, S. B., Dwoskin, L. P., Crooks, P. A., Klebaur, J. E., \& Bardo, M. T. (2001). Lobeline Attenuates $d$-Methamphetamine Self-administration in rats. The Journal of Pharmacology and Experimental Therapeutics, 298(1), 172-179.

Hartz, D. T., Frederick-Osborne, S. L., \& Galloway, G. P. (2001). Craving predicts use during treatment for methapmhetamine dependence: a prospective, repeatedmeasures, within-subject analysis. Drug and Alcohol Dependence, 63, 269-276.

Hauptmann, N., Grimsby, J., Shih, J. C., \& Cadenas, E. (1996). The Metabolism of Tyramine by Monoamine Oxidase A/B Causes Oxidative Damage to Mitochondrial DNA. [doi: DOI: 10.1006/abbi.1996.0510]. Archives of Biochemistry and Biophysics, 335(2), 295-304.

Heffner, T. G., Hartman, J. A., \& Seiden, L. S. (1980). A rapid method for the regional dissection of the rat brain. Pharmacol Biochem Behav, 13, 453-456.

Helkamaa, T., Reenilä, I., Tuominen, R. K., Soinila, S., Väänänen, A., Tilgmann, C., et al. (2007). Increased catechol-O-methyltransferase activity and protein expression in 
OX-42-positive cells in the substantia nigra after lipopolysaccharide microinfusion. [doi: DOI: 10.1016/j.neuint.2007.04.020]. Neurochemistry International, 51(6-7), 412-423.

Hemby, S. E., Co, C., Koves, T. R., Smith, J. E., \& Dworkin, S. I. (1997). Differences in extracellular dopamine concentrations in the nucleus accumbens during responsedependent and response-independent cocaine administration in the rat. Psychopharmacology, 133, 7-16.

Herring, N. R., Schaefer, T. L., Gudelsky, G. A., Vorhees, C. V., \& Williams, M. T. (2008). Effect of (+)-methamphetamine on path integration learning, novel object recognition, and neurotoxicity in rats. Psychopharmacology, 199, 637-650.

Higley, A. E., Kiefer, S. W., Li, X., Gaál, J., Xi, Z.-X., \& Gardner, E. L. (2011). Dopamine D3 receptor antagonist SB-277011A inhibits methamphetamine self-administration and methamphetamine-induced reinstatement of drug-seeking in rats. [doi: DOI: 10.1016/j.ejphar.2011.02.046]. European Journal of Pharmacology, 659, 187-192.

Hiranita, T., Nawata, Y., Sakimura, K., Anggadiredja, K., \& Yamamoto, T. (2006). Suppression of methamphetamine-seeking behaviour by nicotinic agonists. PNAS, $103(22), 8523-8527$.

Hiranita, T., Yamamoto, T., \& Nawata, Y. (2010). A tryptamine-derived catecholaminergic enhancer, (-)-1-(benzofuran-2-yl)-2-propylaminopentane [(-)-BPAP], attenuates reinstatement of methamphetamine-seeking behaviour in rats Neuroscience, 165, 30312.

Hodos, W. (1961). Progressive ratio as a measure of reward strength. Science, 134(3483), 943-944.

Hollander, J. A., \& Carelli, R. M. (2007). Cocaine-associated stimuli increase cocaine seeking and activate accumbens core neurons after abstinence. The Journal of Neuroscience, 27(13), 3535-3539.

Holshneider, D. P., \& Shih, J. C. (2000). Monoamine Oxidase: Basic and clinical perspectives. Psychopharmacology - 4th Generation of Progress, from http://www.acnp.org/g4/GN401000046/Default.htm

Holtzman, S. G. (2001). Differential interaction of GBR12909, a dopmine uptake inhibitor, with cocaine and methamphetamine in rats discriminating cocaine. Psychopharmacology, 155, 180-186.

Hotchkiss, A. J., \& Gibb, J. W. (1980). Long-term effects of multiple doses of methamphetamine on tryptophan hydroxylase and tyrosine hydroxylase activity in rat brain. The Journal of Pharmacology and Experimental Therapeutics, 214, 357-262.

Hotchkiss, A. J., Morgan, M. E., \& Gibb, J. W. (1979). The long-term effect of multiple doses of methamphetamine on neostriatal tryptophan hydroxylase, tyrosine hydroxylase, choline acetyltransferase and glutamate decarboxylase activity. Life Sci, $25,1377-1398$.

Howell, L. L., \& Byrd, L. D. (1995). Serotonergic modulation of the behavioral effects of cocaine in the squirrel monkey. The Journal of Pharmacology and Experimental Therapeutics, 275(3), 1551-1559.

Hubner, C. B., \& Moreton, J. E. (1991). Effects of selective D1 and D2 dopamine antagonists on cocaine self-administration in the rat. Psychopharmacology, 105, 151-156.

Ikemoto, S. (2007). Dopamine reward circuitry: Two projection systems from the ventral midbrain to the nucleus accumbens-olfactory tubercle complex. Brain Research Reviews, 56, 27-78.

Ikemoto, S., Glazier, B. S., Murphy, J. M., \& McBride, W. J. (1997). Role of Dopamine D1 and D2 Receptors in the Nucleus Accumbens in Mediating Reward. J. Neurosci., $17(21), 8580-8587$. 
Imam, S. Z., \& Ali, S. F. (2001). Aging increases the susceptibility to methamphetamineinduced dopaminergic neurotoxicity in rats: correlation with peroxynitrite production and hyperthermia. Journal of Neurochemistry, 78, 952-959.

Ito, R., Dalley, J. W., Robbins, T. W., \& Everitt, B. J. (2002). Dopamine release in the dorsal striatum during cocaine-seeking behavior under the control of a drug-associated cue. The Journal of Neuroscience, 22(14), 6247-6253.

Itzhak, Y., Achat-Mendes, C. (2004). Methamphetamine and MDMA (Ecstasy) Neurotoxicity: 'of Mice and Men'. Life, 56, 249-255.

Izzo, E., Orsini, C., Koob, G. F., \& Pulvirenti, L. (2001). A dopamine partial agonist and antagonist block amphetamine self-administration in a progressive ratio schedule. . Pharmacology, Biochemistry and Behaviour, 68, 701-708.

Jacobs, E. H., Smit, A. B., de Vries, T. J., \& Schoffelmeer, A. N. M. (2003). Neuroadaptive effects of active versus passive drug administration in addiction research. TRENDS in Pharmacological Sciences, 24(11), 566-573.

Johanson, C. E., Balster, R. L., \& Bonese, K. (1976). Self-administration of psychomotor stimulant drugs: the effects of unlimited access. Pharmacol Biochem Behav, 4, 45-51.

Julien, R. M. (2001). A primer of drug action. New York: Henry Holt \& Company.

Jun, J. H., \& Schindler, C. W. (2000). Dextromethorphan alters methamphetamine selfadministration in the rat. Pharmacololgy, Biochemistry and Behavior, 67, 405-409.

Kalivas, P. W., \& McFarland, K. (2003). Brain circuitry and the reinstatement of cocaineseeking behaviour. Psychopharmacology (Berl), 168( ), 44-56.

Katz, J. L., \& Higgins, S. T. (2003). The validity of the reinstatement model of craving and relapse to drug use. Psychopharmacology, 168, 21-30.

Kebabian, J. W., Blanchet, P. J., \& Bedard, P. (1995). The D-1 dopamine receptor: Past, present and future. An Idiosyncrative Review. Prog. Neuro-psychopharmacol. \& Biol. Psychiat., 19, 757-778.

Khroyan, T. V., Barrett-Larimore, R. L., Rowlett, J. K., \& Spealman, R. D. (2000). Dopamine D1- and D2-like Receptor Mechanisms in Relapse to Cocaine-Seeking Behaviour: Effects of Selective Antagonists and Agonists. The Journal of Pharmacology and Experimental Therapeutics, 294(2), 680-687.

Khroyan, T. V., Platt, D. M., Rowlett, J. K., \& Spealman, R. D. (2003). Attenuation of relapse to cocaine seeking by dopamine D1 receptor agonists and antagonists in nonhuman primates. Psychopharmacology (Berl), 168, 124-131.

Kish, S. J. (2008). Pharmacologic mechanisms of crystal meth. CMAJ, 178(13), 1679-1682.

Kitamura, O., Wee, S., Specio, S. E., Koob, G. F., \& Pulvirenti, L. (2006). Escalation of methamphetamine self-administration in rats: a dose-effect function. Psychopharmacology (Berl), 186, 48-53.

Koob, G. F. (2005). The neurocircuitry of addiction: Implications for treatment. Clinical Neuroscinece Research, 5, 89-101.

Koob, G. F. (2006). The neurobiology of addiction: a neuroadaptational view relevant for diagnosis. Addiction, 101, 23-30.

Koob, G. F., \& Le Moal, M. D. (2001). Drug addiction, dysregulation of reward, and allostasis. Neuropsychopharmacology, 24(2), 97-129.

Krasnova, I. N., \& Cadet, J. L. (2009). Methamphetamine toxicity and messengers of death. Brain Research Reviews, 60, 379-407.

Krasnova, I. N., Justinova, Z., Ladenheim, B., Jayanathi, S., McCoy, M. T., Barnes, C., et al. (2010). Methamphetamine Self-administration Is Associated with Persistent Biochemical Alterations in Striatal and Cortical Dopaminergic Terminals in the Rat. PLoS ONE, 5(1), e8790. 
Laurier, L. G., Corrigall, W. A., \& George, S. R. (1994). Dopamine receptor density, sensitivity and mRNA levels are altered following self-administration of cocaine in the rat. Brain Res, 634, 31-40.

Le Merrer, J., Gavello-Baudy, S., Galey, D., \& Cazala, P. (2007). Morphine selfadministration into the lateral septum depends on dopaminergic mechanisms: Evidence from pharmacology and Fos neuroimaging. Behavioural Brain Research, 180(2), 203-217.

Lee, B., London, E. D., Poldrack, R. A., Farahi, J., Nacca, A., Monterosso, J. R., et al. (2009). Striatal dopamine $D_{2} / D_{3}$ receptor availability is reduced in methamphetamine dependence and is linked to impulsivity. The Journal of Neuroscience, 29(47), 1473414740.

Lee, N. K., Pohlman, S., Baker, A., Ferris, J., \& Kay-Lambkin, F. (2010). It's the thought that counts: Craving metacognitions and their role in abstinence from methamphetamine use. Journal of Substance Abuse Treatment, 38(3), 245-250.

Lee, N. K., \& Rawson, R. A. (2008). A systematic review of cognitive and behavioural therapies for methamphetamine dependence. Drug and Alcohol Review, 27, 309-317.

Levey, A. I., Hersch, S. M., Rye, D. B., Sunahara, R. K., Niznik, H. B., Kitt, C. A., et al. (1993). Localization of $\mathrm{D}_{1}$ and $\mathrm{D}_{2}$ receptors in brain with subtype-specific antibodies. Proc. Natl. Acad. Sci. USA, 90, 8861-8865.

Liu, X. a. b., Jernigen, C. a., Gharib, M. b., Booth, S. b., Caggiula, A. R. b., \& Sved, A. F. b. (2010). Effects of dopamine antagonists on drug cue-induced reinstatement of nicotine-seeking behavior in rats. Behavioural Pharmacology, 21(2), 153-160.

Loh, E. A., \& Roberts, D. C. S. (1990). Break-points on a progressive ratio schedule reinforced by intravenous cocaine increase following depletion of forebrain serotonin. Psychopharmacology, 101, 262-266.

Lorez, H. (1981). Fluorescence histochemistry indicates damage of striatal dopamine nerve terminals in rats after multiple doses of methamphetamine. Life Sci, 28, 911-916.

Lynch, W. J., Nicholson, K. L., Dance, M. E., Morgan, R. W., \& Foley, P. L. (2010). Animal models of substance abuse and addiction: Implications for science, animal welfare and society. Comparative Medicine, 60(3), 177-188.

Maeda, H., Sato, T., \& Maki, S. (1985). Effects of dopamine agonists on hypothalamic defensive attack in cats. Physiol Behav, 35, 89-92.

Malmberg, A., \& Mohell, N. (1995). Characterization of [3H]quinpirole binding to human dopamine D2A and D3 receptors: effects of ions and guanine nucleotides. Journal of Pharmacology and Experimental Therapeutics, 274(2), 790-797.

Marinelli, M., Cooper, D. C., Baker, L. K., \& White, F. J. (2003). Impulse actitivity of midbrain dopamine neurons modulates drug-seeking behaviour. Psychopharmacology (Berl), 168, 84-98.

Markou, A. (2009). Accruing preclinical evidence about metabotropic glutamate 5 receptor antagonists as treatments for drug dependence highlights the irreplaceable contributions of animal studies to the discovery of new medications for human disorders. Neuropsychopharmacology, 34(4), 817-819.

Markou, A., Weiss, F., Gold, L. H., Caine, S. B., Schulteis, G., \& Koob, G. F. (1993). Animal models of drug craving. Psychopharmacology, 112, 163-182.

Marsden, C. A. (2006). Dopamine: the rewarding years. British Journal of Pharmacology, 147, S136-S144.

Martelle, J. L., \& Nader, M. A. (2008). A Review of the Discovery, Pharmacological Characterization, and Behavioral Effects of the Dopamine D2-Like Receptor Antagonist Eticlopride. CNS Neurosci Ther, 14(3), 248-262. 
Martin-Negrier, M.-L., Charron, G., \& Bloch, B. (2000). Agonist stimulation provokes dendritic and axonal dopamine D1 receptor redistribution in primary cultures of striatal neurons. Neuroscience, 99(2), 257-266.

Martin, W. R. (1983). Pharmacology of opioids. Pharmacol Rev, 35, 283-323.

Martin, W. R., Sloan, J. W., Sapira, J. D., \& Jasinski, D. R. (1971). Physiologic, subjective, and behavioral effects of amphetamine, methamphetamine, ephedrine, phenmetrazine, and methylphenidate in man. . Clin Pharmacol Ther, 12, 245-258.

Martinez, D., Greene, K., Broft, A., Kumar, D., Liu, F., Narendran, R., et al. (2009). Lower Level of Endogenous Dopamine in Patients With Cocaine Dependence: Findings From PET Imaging of $\mathrm{D}_{2} / \mathrm{D}_{3}$ Receptors Following Acute Dopamine Depletion. The American Journal of Psychiatry, 166(10), 1170.

Martinez, D., Saccone, P. A., Liu, F., Slifstein, M., Orlowska, D., Grassetti, A., et al. (in press). Deficits in Dopamine D2 Receptors and Presynaptic Dopamine in Heroin Dependence: Commonalities and Differences with Other Types of Addiction. [doi: 10.1016/j.biopsych.2011.08.024]. Biological Psychiatry(0).

McGregor, A., \& Roberts, D. C. (1995). Effect of medial prefrontal cortex injections of SCH 23390 on intravenous cocaine self-administration under both a fixed and progressive ratio schedule of reinforcement. Behav Brain Res, 67(1), 75-80.

McGregor, A., \& Roberts, D. C. S. (1993). Dopaminergic antagonism within the nucleus accumbens or the amygdala produces differential effects on intravenous cocaine selfadministration under fixed and progressive ratio schedules of reinforcement. Brain Research, 624(1-2), 245-252.

McLellan, A. T., Kushner, H., Metzger, D., Peters, R., Smith, I., Grissom, G., et al. (1992). The fifth edition of the addiction severity index. [doi: 10.1016/0740-5472(92)90062S]. Journal of Substance Abuse Treatment, 9(3), 199-213.

Melega, W. P., Jorgensen, M. J., Laćan, G., Way, B. M., Pham, J., Morton, G., et al. (2008). Long-Term Methamphetamine Administration in the Vervet Monkey Models Aspects of a Human Exposure: Brain Neurotoxicity and Behavioural Profiles. Neuropsychopharmacology, 33, 1441-1452.

METHCON. (2008). Solutions to the methamphetamine crisis in New Zealand: A study of supply and demand-side interventions and their efficacy.

Milivojevič, N., Krisch, I., Sket, D., \& Živin, M. (2004). The dopamine D 1 $_{1}$ receptor agonist and $\mathrm{D}_{2}$ receptor antagonist LEK-8829 attenuates reinstatement of cocaine-seeking in rats. Naunyn-Schmiedeberg's Archives of Pharmacology, 369(6), 576-582.

Millan, M. J., Newman-Tancredi, A., Quentric, Y., \& Cussac, D. (2001). The "selective" dopamine $\mathrm{D}_{1}$ receptor antagonist, $\mathrm{SCH} 23390$, is a potent and high efficacy agonist at cloned human serotonin 2 receptors. Psychopharmacology (Berl), 156, 58-62.

Missale, C., Nash, S. R., Robinson, S. W., Jaber, M., \& Caron, M. G. (1998). Dopamine receptors: From structure to function. Physiological Reviews, 78(7), 189-225.

Miszkiel, J., Filip, M., \& Przegaliński, E. (2011). Role of serotonin (5-HT)(1B) receptors in psychostimulant addiction. Pharmacol Rep, 63(6), 1310-1315.

Moffett, M. C., \& Goeders, N. E. (2007). CP-154,526, a CRF type-1 receptor antagonist, attenuates the cue- and methamphetamine-induced reinstatement of extinguished methamphetamine-seeking behaviour in rats. Psychopharmacology, 190, 171-180.

Morgan, D., Grant, K. A., Gage, H. D., Mach, R. H., Kaplan, J. R., Prioleau, O., et al. (2002). Social dominance in monkeys: dopamine D2 receptors and cocaine selfadministration. Nature Neuroscience, 5(2), 169-174.

Mucha, R. F., \& Herz, A. (1985). Motivational properties of kappa and mu opioid receptor agonists studied with place and taste preference conditioning. Psychopharmacology, 86(3), 274-280. 
Müller, C. P., Carey, R. J., Huston, J. P., \& De Souza Silva, M. A. (2007). Serotonin and psychostimulant addiction: Focus on 5- $\mathrm{HT}_{1 \mathrm{~A}}$-receptors. Progress in Neurobiology, 81, 133-178.

Munzar, P., Baumann, M. H., Shoaib, M., \& Goldberg, S. R. (1999). Effects of dopamine and serotonin-releasing agents on methamphetamine discrimination and selfadministration in rats. Psychopharmacology (Berl), 141, 287-296.

Nader, M. A., Daunais, J. B., Moore, T., Nader, S. H., Moore, R. J., Smith, H. R., et al. (2002). Effects of cocaine self-administration on striatal dopamine systems in rhesus monkeys: Initial and chronic exposure. Neuropsychopharmacology, 27(1), 35-46.

Neisewander, J. L., O'Dell, L. E., Tran-Nguyen, L. T. L., Castañeda, E., \& Fuchs, R. A. (1996). Dopamine overflow in the nucleus accumbens during extinction and reinstatement of cocaine self-administration behaviour. Neuropsychopharmacology, $15(5), 506-514$.

Neugebauer, N. M., Harrod, S. B., Stairs, D. J., Crooks, P. A., Dwoskin, L. P., Bardo, M. T. (2007). Lobelane decreases methamphetamine self-administration in rats. European Journal of Pharmacology, 571, 33-38.

Newton, T. F., De La Garza II, R., Kalechstein, A. D., \& Nestor, L. (2005). Cocaine and methamphetamine produce different patterns of subjective and cardiovascular effects. Pharmacology, Biochemistry and Behaviour, 82, 90-97.

Nickell, J. R., Krishnamurthy, S., Norrholm, S., Deaciuc, G., Siripurapu, K. B., Zheng, G., et al. (2010). Lobelane inhibits methamphetamine-evoked dopamine release via inhibition of the vesicular monoamine transporter-2. The Journal of Pharmacology and Experimental Therapeutics, 332(2), 612-621.

Nicosia, N., Pacula, R. L., Kilmer, B., Lundberg, R., \& Chiesa, J. (2009). The economic cost of methamphetamine use in the United States, 2005. Santa Monica, CA: Drug Policy Research Centre, RAND Corp.

NIDA. (2006). Methamphetamine abuse and addiction. Bethesda, MD: NIH, DHHS.

NSDUH. (2007). The National Survey on Drug Use and Health: Methamphetamine use: Substance Abuse and Mental Health Services Administration.

O'Dell, S. J., \& Marshall, J. F. (2005). Neurotoxic regimens of methamphetamine induce persistent expression of phospho-c-Jun in somatosensory cortex and substantia nigra. Synapse, 55(3), 137-147.

O'Neil, M. L., Kuczenski, R., Segal, D. S., Cho, A. K., Lacan, G., \& Melega, W. P. (2006). Escalating dose pretreatment induces pharmacodynamic and not pharmacokinetic tolerance to a subsequent high-dose methamphetamine binge. Synapse, 60(6), 465473.

Olive, M. F. (2005). mGlu5 receptors: Neuroanatomy, pharmacology, and role in drug addiction. Current Psychiatry Reviews, 1(2), 197-214.

Orio, L., Wee, S., Newman, A. H., Pulvirenti, L., \& Koob, G. F. (2010). The dopamine D3 receptor partial agonist CJB090 and antagonist PG01037 decrease progressive ratio responding for methamphetamine in rats with extended access. Addict Biol, 15(3), 312-323.

Panlilio, L. V., \& Goldberg, S. R. (2007). Self-administration of drugs in animals and humans as a model and an investigative tool. Addiction, 102, 1863-1870.

Partilla, J. S., Dempsey, A. G., Nagpal, A. S., Blough, B. E., Baumann, M. H., \& Rothman, R. B. (2006). Interaction of amphetamines and related compounds at the vesicular monoamine transporter. The Journal of Pharmacology and Experimental Therapeutics, 319(1), 237-246.

Patton, J. H., Stanford, M. S., \& Barratt, E. S. (1995). Factor structure of the Barratt impulsiveness scale. J Clin Psychol, 51, 768-774. 
Peris, J., Boyson, S. J., Cass, W. A., Curella, P., Dwoskin, L. P., Larson, G., et al. (1990). Persistence of neurochemical changes in dopamine systems after repeated cocaine administration. The Journal of Pharmacology and Experimental Therapeutics, 253(1), 38-44.

Phillips, G. D., Robbins, T. W., \& Everitt, B. J. (1994). Bilateral intra-accumbens selfadministration of $d$-amphetamine: Antagonism with intra-accumbens SCH-23390 and sulpiride. Psychopharmacology, 114(3), 477-485.

Pontieri, F. E., Tanda, G., \& Di Chiara, G. (1995). Intravenous cocaine, morphine, and amphetamine preferentially increase extracellular dopamine in the "shell" as compared with the "core" of the rat nucleus accumbens. Proc. Natl. Acad. Sci. USA, 92, 12304-12308.

Pubill, D., Canudas, A. M., Pallás, M., Camins, P., Camarasa, J., \& Escubedo, E. (2003). Different glial response to methamphetamine- and methylenedioxymethamphetamineinduced neurotoxicity. Naunyn-Schmiedeberg's Archives of Pharmacology, 367, 490499.

Pulvirenti, L., Balducci, C., Piercy, M., \& Koob, G. F. (1998). Characterization of the effects of the partial dopamine agonist terguride on cocaine self-administration in the rat. The Journal of Pharmacology and Experimental Therapeutics, 286(3), 1231-1238.

Quinton, M. S., \& Yamamoto, B. K. (2006). Causes and consequences of methamphetamine and MDMA toxicity. The AAPS Journal, 8(2), E337-E347.

Raiteri, M., Cerrito, F., \& Cervoni, A. M. (1979). Dopamine can be released by two mechanisms differentially affected by the dopamine transport inhibitor nomifensine. The Journal of Pharmacology and Experimental Therapeutics, 208, 195-202.

Ranaldi, R., \& Poeggel, K. (2002). Baclofen decreases methamphetamine self-administration in rats. NeuroReport, 13(9), 1107-1110.

Ranaldi, R., \& Wise, R. A. (2001). Blockade of D1 Dopamine Receptors in the Ventral Tegmental Area Decreases Cocaine Reward: Possible Role for Dendritically Released Dopamine. The Journal of Neuroscience, 21(15), 5841-5846.

Ricaurte, G. A., Guillery, R. W., Seiden, L. S., Schuster, C. R., \& Moore, R. Y. (1982). Dopamine nerve terminal degeneration produced by high doses of methylamphetamine in the rat brain. Brain Res, 235, 93-103.

Ricaurte, G. A., Schuster, C. R., \& Seiden, L. S. (1980). Long-term effect of repeated methamphetamine administration on dopamine and serotonin neurons in rat brain: A regional study. Brain Res, 193, 153-160.

Richardson, N. R., \& Roberts, D. C. S. (1996). Progressive ratio schedules in drug selfadministration studies in rats: a method to evaluate reinforcing efficacy. Journal of Neuroscience Methods, 66(1), 1-11.

Ritz, M. C., Cone, E. J., \& Kuhar, M. J. (1990). Cocaine inhibition of ligand binding at dopamine, norepinephrine and serotonin transporters: a structure-activity study. Life Sci, 46, 635-645.

Roberts, D. C. S. (1993). Self-administration of GBR 12909 on a fixed ratio and progressive ratio schedule in rats. Psychopharmacology (Berl), 111(2), 202-206.

Roberts, D. C. S., Andrews, M. M., \& Vickers, G. J. (1996). Baclofen attenuates the reinforcing effects of cocaine in rats. Neuropsychopharmacology, 15(4), 417-423.

Roberts, D. C. S., Morgan, D., \& Liu, Y. (2007). How to make a rat addicted to cocaine. Prog. Neuro-psychopharmacol. \& Biol. Psychiat., 31, 1614-1624.

Robinson, T. E., \& Camp, D. M. (1987). Long-lasting effects of escalating doses of damphetamine on brain monoamines, amphetamine-induced stereotyped behavior and spontaneous nocturnal locomotion. Pharmacol Biochem Behav, 26(4), 821-827. 
Rogers, J. L., De Santis, S., \& See, R. E. (2008). Extended methamphetamine selfadministration enhances reinstatement of drug seeking and impairs novel object recognition in rats. Psychopharmacology (Berl), 199(4), 615-624.

Roth, M. E., \& Carroll, M. E. (2004). Sex differences in the acquisition of IV methamphetamine self-administration and subsequent maintenance under a progressive ratio schedule in rats. Psychopharmacology (Berl), 172, 443-449.

Rothman, R. B., \& Baumann, M. H. (2006). Balance between dopamine and serotonin release modulates behavioral effects of amphetamine-type drugs. Ann. N.Y. Acad. Sci., 1074, 245-260.

Rothman, R. B., Baumann, M. H., Dersch, C. M., Romero, D. V., Rice, K. C., Carroll, F. I., et al. (2001). Amphetamine-type central nervous system stimulants release norepinephrine more potently than they release dopamine and serotonin. Synapse, 39(1), 32-41.

Rowlett, J. K., Massey, B. W., Kleven, M. S., \& Woolverton, W. L. (1996). Parametric analysis of cocaine self-administration under a progressive-ratio schedule in rhesus monkeys. Psychopharmacology, 125(4), 361-370.

Sanchis-Segura, C., \& Spanagel, R. (2006). Behavioural assessment of drug reinforcement and addictive features in rodents: an overview. Addiction Biology, 11, 2-38.

Schenk, S., \& Gittings, D. (2003). Effects of SCH 23390 and eticlopride on cocaine-seeking produced by cocaine and WIN 35,428 in rats. Psychopharmacology (Berl), 168, 118123.

Schenk, S., Gittings, D., \& Colussi-Mas, J. (2011). Dopaminergic mechanisms of reinstatement of MDMA-seeking behaviour in rats. British Journal of Pharmacology, $162,1770-1780$.

Schenk, S., Gittings, D., Johnstone, M., \& Daniela, E. (2003). Development, maintenance and temporal pattern of self-administration maintained by ecstasy (MDMA) in rats. Psychopharmacology, 169, 21-27.

Schenk, S., Hely, L., Gittings, D., Lake, B., \& Daniela, E. (2008). Effects of priming injections of MDMA and cocaine on reinstatement of MDMA- and cocaine-seeking in rats. Drug and Alcohol Dependence, 96(3), 249-255.

Schenk, S., Hely, L., Lake, B., Daniela, E., Gittings, D., \& Mash, D. C. (2007). MDMA selfadministration in rats: acquisition, progressive ratio responding and serotonin transporter binding. European Journal of Pharmacology, 26, 3229-3236.

Schenk, S., \& Partridge, B. (1999). Cocaine-seeking produced by experimenter-administered drug injections: dose-effect relationships in rats. Psychopharmacology, 147(3), 285290.

Schmidt, C. J., Sonsalla, P. K., Hanson, G. R., Peat, M. A., \& Gibb, J. W. (1985). Methamphetamine-induced depression of monoamine synthesis in the rat: development of tolerance. J Neurochem, 44, 852-855.

Schmidt, H. D., \& Pierce, R. C. (2006). Systemic administration of a dopamine, but not a serotonin or norepinephrine, transporter inhibitor reinstates cocaine seeking in the rat. Behavioural Brain Research, 175, 189-194.

Schmitt, K. C., \& Reith, M. E. A. (2010). Regulation of the dopamine transporter: Aspects relevant to psychostimulant drugs of abuse. Annals of the New York Academy of Sciences, 1187, 316-340.

Schuster, C. R., \& Thompson, T. (1969). Self Administration of and Behavioural Dependence on Drugs. Annu. Rev. Pharmacol., 9, 483-502.

Schwendt, M., Rocha, A., See, R. E., Pacchioni, A. M., McGinty, J. F., \& Kalivas, P. W. (2009). Extended Methamphetamine Self-Administration in Rats Results in a Selective Reduction of Dopamine Transporter Levels in the Prefrontal Cortex and 
Dorsal Striatum Not Accompanied by Marked Monoaminergic Depletion. Journal of Pharmacology and Experimental Therapeutics, 331(2), 555-562.

See, R. E., Elliott, J. C., \& Feltenstein, M. W. (2007). The role of dorsal vs ventral striatal pathways in cocaine-seeking behaviour after prolonged abstinence in rats. Psychopharmacology (Berl), 194, 321-331.

Seeman, P., McCormick, P. N., \& Kapur, S. (2007). Increased dopamine D2 ${ }^{\text {high }}$ receptors in amphetamine-sensitized rats, measured by the agonist $\left[{ }^{3} \mathrm{H}\right](+)$ PHNO. Synapse, 61, 263-267.

Seeman, P., \& Schaus, J. M. (1991). Dopamine receptors labelled by [3H] quinpirole. [doi: 10.1016/0014-2999(91)90796-S]. European Journal of Pharmacology, 203(1), 105109.

Seeman, P., Tallerico, T., Ko, F., Tenn, C., \& Kapur, S. (2002). Amphetamine-sensitized animals show a marked increase in dopamine $\mathrm{D}_{2}$ high receptors occupied by endogenous dopamine, even in the absence of acute challenges. Synapse, 46, 235-239.

Segal, D. S., Kuczenski, R., O'Neil, M. L., Melega, W. P., \& Cho, A. K. (2003). Escalating dose methamphetamine pretreatment alters the behavioral and neurochemical profiles associated with exposure to a high-dose methamphetamine binge. Neuropsychopharmacology, 28(10), 1730-1740.

Segal, D. S., Kuczenski, R., O'Neill, M. L., Melega, W. P., \& Cho, A. K. (2005). Prolonged exposure of rats to intravenous methamphetamine: behavioral and neurochemical characterization. Psychopharmacology (Berl), 180, 501-512.

Self, D. W. (1998). Neural substrates of drug craving and relapse in drug addiction. Ann Med, 30, 379-389.

Self, D. W. (2004a). Drug Dependence and Addiction. The American Journal of Psychiatry, 161(2), 223.

Self, D. W. (2004b). Regulation of drug-taking and -seeking behaviours by neuroadaptations in the mesolimbic dopamine system. Neuropharmacology, 47, 242-255.

Self, D. W., Barnhart, W. J., Lehman, D. A., \& Nestler, E. J. (1996). Opposite modulation of cocaine-seeking behavior by $\mathrm{D}_{1^{-}}$and $\mathrm{D}_{2}$-like dopamine receptor agonists. Science, $271,1586-1589$.

Self, D. W., Belluzzi, J., Kossuth, S., \& Stein, L. (1996). Self-administration of the $\mathrm{D}_{1}$ agonist SKF 82958 is mediated by $\mathrm{D}_{1}$, not $\mathrm{D}_{2}$, receptors. Psychopharmacology, 123(4), 303-306.

Self, D. W., \& Nestler, E. J. (1998). Relapse to drug-seeking: neural and molecular mechanisms. [doi: DOI: 10.1016/S0376-8716(98)00065-9]. Drug and Alcohol Dependence, 51(1-2), 49-60.

Shaham, Y., \& Miczek, K. A. (2003). Reinstatement - toward a model of relapse. Psychopharmacology (Berl), 168, 1-2.

Shaham, Y., Shalev, U., Lu, L., de Wit, H., \& Stewart, J. (2003). The reinstatement model of drug relapse: history, methodology and major findings. Psychopharmacology, 168, 320.

Shaham, Y., \& Stewart, J. (1996). Effects of opioid and dopamine receptor antagonists on relapse induced by stress and re-exposure to heroin in rats. Psychopharmacology (Berl), 125, 385-391.

Shalev, U., Grimm, J. W., \& Shaham, Y. (2002). Neurobiology of relapse to heroin and cocaine seeking: a review. Pharmacological Reviews, 54, 1-42.

Sharma, H. S., \& Kiyatkin, E. A. (2009). Rapid morphological brain abnormalities during acute methamphetamine intoxication in the rat: An experimental study using light and electron microscopy. [doi: 10.1016/j.jchemneu.2008.08.002]. Journal of Chemical Neuroanatomy, 37(1), 18-32. 
Shelton, K. L., \& Beardsley, P. M. (2008). Effect of drug-paired exteroceptive stimulus presentations on methamphetamine reinstatement in rats. Pharmacology, Biochemistry and Behaviour, 90, 434-440.

Shepard, J. D., Bossert, J. M., Liu, S. Y., \& Shaham, Y. (2004). The anxiogenic drug yohimbine reinstates methamphetamine seeking in a rat model of drug relapse. Biol Psychiatry, 55, 1082-1089.

Shepard, J. D., Chuang, D. T., Shaham, Y., \& Morales, M. (2006). Effect of methamphetamine self-administration on tyrosine hydroxylase and dopamine transporter levels in mesolimbic and nigrostrital dopamine pathways of the rat. Psychopharmacology (Berl), 185, 505-513.

Shintomi, K. (1975). Effects of psychotropic drugs on methamphetamine-induced behavioral excitation in grouped mice. European Journal of Pharmacology, 31, 195-206.

Shishido, T., Watanabe, Y., Suzuki, H., Kato, K., Niwa, A., Hanoune, J., et al. (1997). Effects of repeated methamphetamine administration on dopamine D1 receptor, D2 receptor and adenylate cyclase type V mRNA levels in the rat striatum. Neuroscience Letters, 222, 175-178.

Shuto, T., Kuroiwa, M., Hamamura, M., Yabuuchi, K., Shimazoe, T., Watanabe, S., et al. (2006). Reversal of methamphetamine-induced behavioral sensitization by repeated administration of a dopamine $\mathrm{D}_{1}$ receptor agonist. Neuropharmacology, 50, 991-997.

Skjoldager, P., Winger, G., \& Woods, J. H. (1991). Analysis of fixed-ratio behaviour maintained by drug reinforcers. Journal of the experimental analysis of behaviour, 56(2), 331-343.

Smiley, J. F., Levey, A. I., Ciliax, B. J., \& Goldman-Rakic, P. S. (1994). D1 dopamine receptor immunoreactivity in human and monkey cerebral cortex: Predominant and extrasynaptic localization in dendritic spines. . Proc. Natl. Acad. Sci. USA, 91, 57205724.

Smith, J. E., Co, C., Freeman, M. E., \& Lane, J. D. (1982). Brain neurotransmitter turnover correlated with morphine-seeking behavior of rats. Pharmacology, Biochemistry and Behavior, 16(3), 509-519.

Spealman, R. D., \& Goldberg, S. R. (1978). Drug Self-Administration by Laboratory Animals: Control by Schedules of Reinforcement. Ann. Rev. Pharmacol. Toxicol., 18, 313-339.

Stafford, D., LeSage, M. G., \& Glowa, J. R. (1998). Progressive-ratio schedules of drug delivery in the analysis of drug self-administration: a review. Psychopharmacology (Berl), 139, 169-184.

Stefanski, R., Justinova, Z., Hayashi, T., Takebayashi, M., Goldberg, S. R., \& Su, T. (2004). Sigma $_{1}$ receptor upregulation after chronic methamphetamine self-administration in rats: a study with yoked controls. Psychopharmacology, 175, 68-75.

Stefanski, R., Ladenheim, B., Lee, S. H., Cadet, J. L., \& Goldberg, S. R. (1999). Neuroadaptations in the dopaminergic system after active self-administration but not after passive administration of methamphetamine. European Journal of Pharmacology, 371, 123-135.

Stefanski, R., Lee, S., Yasar, S., Cadet, J. L., \& Goldberg, S. R. (2002). Lack of persistent changes in the dopaminergic system of rats withdrawn from methamphetamine selfadministration. European Journal of Pharmacology, 439, 59-68.

Stefanski, R., Ziółkowska, B., Kuśmider, M., Mierzejeski, P., Wyszogrodska, E., Kołomańska, P., et al. (2007). Active versus passive cocaine administration: Differences in the neuroadaptive changes in the brain dopaminergic system. Brain Research, 1157, 1-10. 
Stephans, S., \& Yamamoto, B. (1996). Methamphetamine pretreatment and the vulnerability of the striatum to methamphetamine neurotoxicity. Neuroscience, 72(3), 593-600.

Stephens, D. N., Duka, T., Crombag, H. S., Cunningham, C. L., \& Heilig, M. (2010). Reward sensitivity: issues of measurement, and achieving consilience between human and animal phenotypes. Addiction Biology, 15, 145-168.

Stewart, J. (1983). Conditioned and unconditioned drug effects in relapse to opiate and stimulant drug self-adminstration. Prog Neuropsychopharmacol Biol Psychiatry, 7, 591-597.

Stewart, J. (1984). Reinstatement of heroin and cocaine self-administration behavior in the rat by intracerebral application of morphine in the ventral tegmental area. Pharmacol Biochem Behav. , 20(6), 917-923.

Stewart, J. (2000). Pathways to relapse: the neurobiology of drug- and stress-induced relapse to drug-taking. Journal of Psychiatry \& Neuroscience 25(2), 125-136.

Stretch, R., \& Gerber, G. J. (1973). Drug-induced reinstatement of amphetamine selfadministration behaviour in monkeys. Canad. J. Psychol., 27(2), 168-177.

Sulzer, D. (2001). How addictive drugs disrupt presynaptic dopamine neurotransmission. Neuron, 69, 628-649.

Sulzer, D., Sonders, M. S., Poulsen, N. W., \& Galli, A. (2005). Mechanisms of neurotransmitter release by amphetamines: A review. [doi: 10.1016/j.pneurobio.2005.04.003]. Progress in Neurobiology, 75(6), 406-433.

Suzuki, O., Hattori, H., Asano, M., Oya, M., \& Katsumata, Y. (1980). Inhibition of monoamine oxidase by d-methamphetamine. [doi: 10.1016/0006-2952(80)90493-1]. Biochemical Pharmacology, 29(14), 2071-2073.

Tella, S. R. (1995). Effects of monoamine reuptake inhibitors on cocaine self-administration in rats. Pharmacology Biochemistry and Behavior, 51(4), 687-692.

Teng, L., Crooks, P. A., Sonsalla, P. K., \& Dwoskin, L. P. (1997). Lobeline and nicotine evoke $\left[{ }^{3} \mathrm{H}\right]$ overflow from rat striatal slices preloaded with $\left[{ }^{3} \mathrm{H}\right]$ dopamine: Differential inhibition of synaptosomal and vesicular $\left[{ }^{3} \mathrm{H}\right]$ dopamine uptake. The Journal of Pharmacology and Experimental Therapeutics, 280(3), 1432-1444.

Tessel, R. E., \& Woods, J. H. (1975). Fenfluramine and N-ethyl amphetamine: Comparison of the reinforcing and rate-decreasing actions in the rhesus monkey. Psychopharmacologia, 43, 239-244.

Thomas, D. M., Francescutti-Verbeem, D. M., \& Kuhn, D. M. (2008). The newly synthesized pool of dopamine determines the severity of methamphetamine-induced neurotoxicity. Journal of Neurochemistry, 105, 605-616.

Tran-Nguyen, L. T. L., Fuchs, R. A., Coffey, G. P., Baker, D. A., O'Dell, L. E., \& Neisewander, J. L. (1998). Time-dependent changes in cocaine-seeking behaviour and extracellular dopamine levels in the amygdala during cocaine withdrawal. Neuropsychopharmacology, 19(1), 48-59.

Twining, R. C., Bolan, M., \& Grigson, P. S. (2009). Yoked delivery of cocaine is aversive and protects against the motivation for drug in rats. Behavioral Neuroscience, 123(4), 913-925.

Tziortzis, D., Mahoney Iii, J. J., Kalechstein, A. D., Newton, T. F., \& La Garza Ii, R. D. (2011). The relationship between impulsivity and craving in cocaine- and methamphetamine-dependent volunteers. [doi: 10.1016/j.pbb.2010.12.022]. Pharmacology Biochemistry and Behavior, 98(2), 196-202.

UNODC. (2010). United Nations Office on Drugs and Crime: World Drug Report.

Vanderschuren, L. J. M. J., Di Ciano, P., \& Everitt, B. J. (2005). Involvement of the dorsal striatum in cue-controlled cocaine seeking. The Journal of Neuroscience, 25(38), 8665-8670. 
Volkow, N. D., Chang, J., Wang, G., Fowler, J. S., Ding, Y., Sedler, M., et al. (2001). Low level of brain dopamine $\mathrm{D}_{2}$ receptors in methamphetamine abusers: Association with metabolism in the orbitofrontal cortex. Am J Psychiatry, 158, 2015-2021.

Volkow, N. D., \& Fowler, J. S. (2000). Addiction, a disease of compulsion and drive: involvement of the orbitofrontal cortex. Cerebral Cortex, 10, 318-325.

Volkow, N. D., Fowler, J. S., Wang, G., Hitzemann, R., Logan, J., Schlyer, D. J., et al. (1993). Decreased dopamine D2 receptor availability is associated with reduced frontal metabolism in cocaine abusers. Synapse, 14, 169-177.

Volkow, N. D., Wang, G., Fowler, J. S., Logan, J., Gatley, S. J., Gifford, A., et al. (1999). Prediction of reinforcing responses to psychostimulants in humans by brain dopamine D2 receptor levels. The American Journal of Psychiatry, 156(9), 1440-1443.

Volkow, N. D., Wang, G., Telang, F., Fowler, J. S., Logan, J., Childress, A., et al. (2006). Cocaine cues and dopamine in dorsal striatum: Mechanism of craving in cocaine addiction. The Journal of Neuroscience, 26(24), 6583-6588.

Volz, T. J., Farnsworth, S. J., Rowley, S. D., Hanson, G. R., \& Fleckenstein, A. E. (2009). Age-dependent differences in dopamine transporter and vesicular monoamine transporter-2 function and their implications for methamphetamine neurotoxicity. Synapse, 63(2), 147-151.

Waddington, J. L., Daly, S. A., Downes, R. P., Deveney, A. M., McCauley, P. G., \& O'Boyle, K. M. (1995). Behavioural pharmacology of 'D-1-like' dopamine receptors: further subtyping, new pharmacalogical probes and interactions with 'D-2-like' receptors. Prog. Neuro-psychopharmacol. \& Biol. Psychiat., 19, 811-831.

Wagner, G. C., Ricaurte, G. A., Seiden, L. S., Schuster, C. R., Miller, R. J., \& Westley, J. (1980). Long-lasting depletions of striatal dopamine and loss of dopamine uptake sites following repeated administration of methamphetamine. [doi: DOI: 10.1016/00068993(80)91265-2]. Brain Research, 181(1), 151-160.

Wallace, D. R., Mactutus, C. F., \& Booze, R. M. (1996). Repeated Intravenous Cocaine Administration: Locomotor Activity and Dopamine $\mathrm{D}_{2} / \mathrm{D}_{3}$ Receptors. Synapse, 23, $152-463$.

Wallace, T. L., Gudelsky, G. A., \& Vorhees, C. V. (2001). Neurotoxic regimen of methamphetamine produces evidence of behavioral sensitization in the rat. Synapse, $39(1), 1-7$.

Wang, Z., \& Woolverton, W. L. (2007). Estimating the relative reinforcing strength of ( \pm )3,4-methylenedioxymethamphetamine (MDMA) and its isomers in rhesus monkeys: comparison to (+)-methamphetamine. Psychopharmacology, 189, 483-488.

Wee, S., Wang, Z., He, R., Zhou, J., Kozikowski, A. P., \& Woolverton, W. L. (2006). Role of the increased noradrenergic neurotransmission in drug self-administration. Drug and Alcohol Dependence, 82, 151-157.

Wee, S., Wang, Z., Woolverton, W. L., Pulvirenti, L., \& Koob, G. F. (2007). Effect of Aripiprazole, a Partial Dopamine D2 Receptor Agonist, on Increased Rate of Methamphetamine Self-Administration in Rats with Prolonged Session Duration. Neuropsychopharmacology, 32, 2238-2247.

Weed, M. R., \& Woolverton, W. L. (1995). The reinforcing effects of dopamine D1 receptor agonists in rhesus monkeys. The Journal of Pharmacology and Experimental Therapeutics, 275(3), 1367-1374.

Weeks, J. R. (1962). Experimental morphine addiction: Method for autonomic intravenous injections in unrestrained rats. Science, 138, 143-144.

Wei, Q., Yeung, M., Jurma, O. P., \& Andersen, J. K. (1996). Genetic elevation of monoamine oxidase levels in dopaminergic PC12 cells results in increased free 
radical damage and sensitivity to MPTP. Journal of Neuroscience Research, 46(6), 666-673.

Weinshenker, D., \& Schroeder, J. P. (2007). There and back again: A tale of norepinephrine and drug addiction. Neuropsychopharmacology, 32, 1433-1451.

Weiss, F., Maldonado-Vlaar, C. S., Parsons, L. H., Kerr, T. M., Smith, D. L., \& Ben-Shahar, O. (2000). Control of cocaine-seeking behaviour by drug-associated stimuli in rats: Effects on recovery of extinguished operant-responding and extracellular dopamine levels in amygdala and nucleus accumbens. Proc. Natl. Acad. Sci. USA, 97(8), 43214326.

Wilhelm, C. J., Johnson, R. A., Eshleman, A. J., \& Janowsky, A. (2008). Lobeline effects on tonic and methamphetamine-induced dopamine release. Biochemical Pharmacology, $75,1411-1415$.

Wilkins, C., \& Rose, E. (2004). The socio-economic impact of amphetamine type stimulants (ATS) in New Zealand: Part III - Future trends in the use and supply of methamphetamine. Auckland: Massey University.

Wilson, M. C., Hitomi, M., \& Schuster, C. R. (1971). Psychomotor stimulant self administration as a function of dosage per injection in the rhesus monkey. Psychopharmacology (Berl.), 22, 271-281.

Wilson, M. C., \& Schuster, C. R. (1972). The effects of chlorpromazine on psychomotor stimulant self-administration in the rhesus monkey. Psychopharmacology, 26, 115126.

Wise, R. A., \& Bozarth, M. A. (1987). A psychomotor stimulant theory of addiction. Psychological Review, 94(4 ), 469-492.

Wise, R. A., Leeb, K., Pocock, D., Newton, P., Burnette, B., \& Justice, J. B. (1995). Fluctuations in nucleus accumbens dopamine concentration during intravenous cocaine self-administration in rats. Psychopharmacology, 120(1), 10-20.

Wise, R. A., Murray, A., \& Bozarth, M. A. (1990). Bromocriptine self-administration and bromocriptine-reinstatement of cocaine-trained and heroin-trained lever pressing in rats. Psychopharmacology (Berl), 100, 355-360.

Wise, R. A., \& Rompre, P. P. (1989). Brain Dopamine and Reward. Ann. Rev. Psychol. , 40, 191-225.

Xi, Z. X., Kleitz, H. K., Deng, X., Ladenheim, B., Peng, X. Q., Li, X., et al. (2009). A single high dose of methamphetamine increases cocaine self-administration by depletion of striatal dopamine in rats. [doi: DOI: 10.1016/j.neuroscience.2009.03.060]. Neuroscience, 161(2), 392-402.

Yahyavi-Firouz-Abadi, N., \& See, R. E. (2009). Anti-relapse medications: Preclinical models for drug addiction treatment. Pharmacology \& Therapeutics, 124, 235-247.

Yokel, R. A., \& Pickens, R. (1973). Self-administration of optical isomers of amphetamine and methylamphetamine by rats. The Journal of Pharmacology and Experimental Therapeutics, 187(1), 27-33.

Yokel, R. A., \& Wise, R. A. (1976). Attenuation of intravenous amphetamine reinforcement by central dopamine blockade in rats. Psychopharmacology, 48, 311-318.

Zaczek, R., Culp, S., \& De Souza, E. B. (1991). Interactions of $\left[{ }^{3} \mathrm{H}\right]$ Amphetamine with rat brain synaptosomes. II. Active Transport. The Journal of Pharmacology and Experimental Therapeutics, 257(2), 830-835.

Zhang, D., Wang, X., Xiang, X., Chen, H., Zhang, J., Su, Q., et al. (2010). The dopamine D2 partial agonist and antagonist terguride decreases heroin self-administration on fixedand progressive-ratio schedules. Pharmacology Biochemistry and Behavior, 97(2), 222-226. 
Zhang, Y., Loonam, T. M., \& Noailles, P. A. H. (2001). Comparison of cocaine- and methamphetamine-evoked dopamine and glutamate overflow in somatodendritic and terminal field regions of the rat brain during acute, chronic, and early withdrawal condtions. Annals of the New York Academy of Sciences, 937, 93-120. 